19

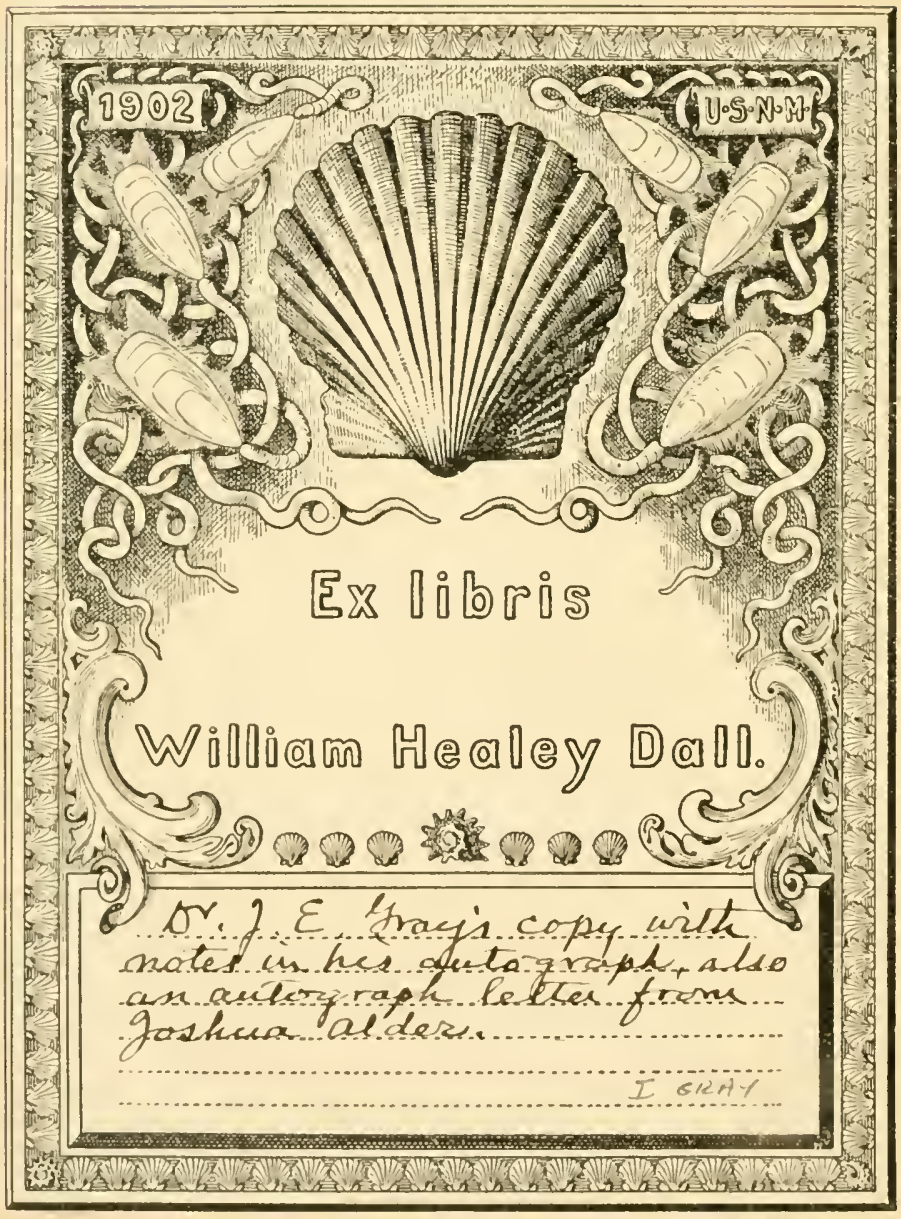


De

466

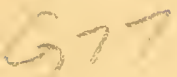

1850

Mow 



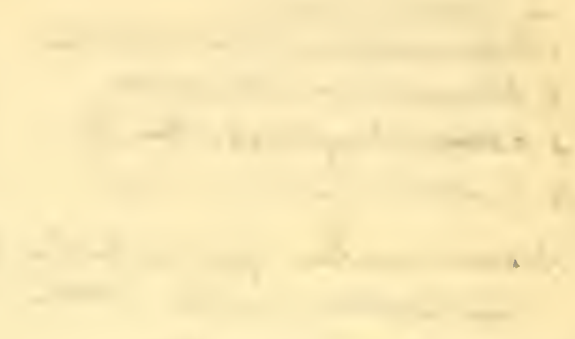

$\rightarrow$

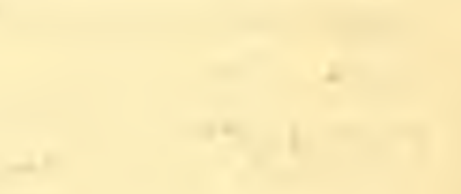


Yren- 


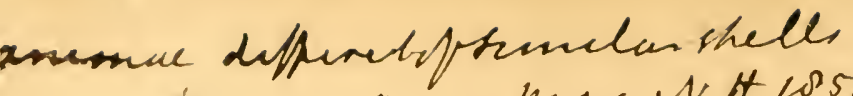
trenson an mag os it $205 \% 37\rangle$ may

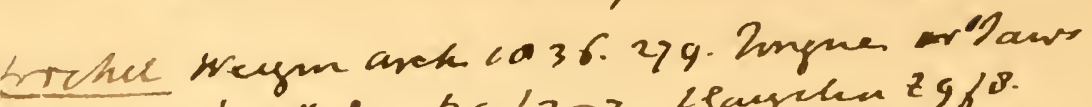

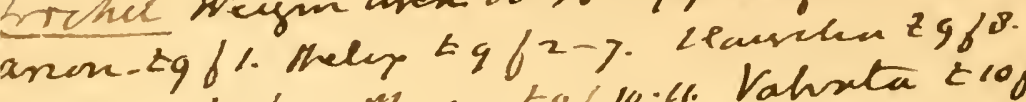

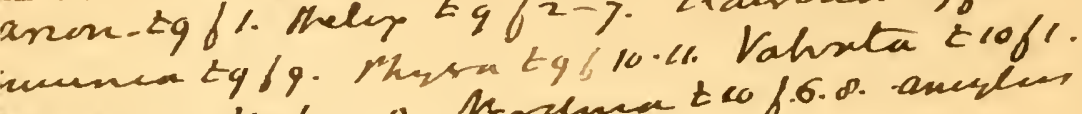

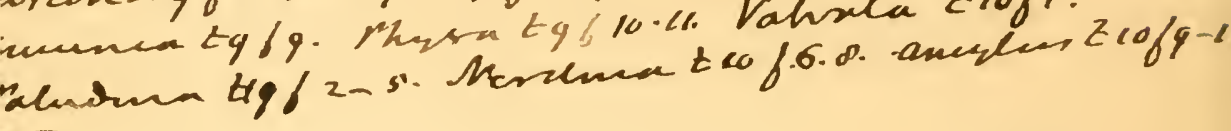

I GRNY 


\section{SYSTEMATIC ARRANGEMENT OF THE}

\section{FIGURES.}

THE genera are chiefly arranged according to the "List of the Genera of Recent Mollusea, their synonyma and types," printed in the Proceedings of the Zoological Society for 1847, a copy of whieh accompanies this volume. The number after the generic name refers to the number in the List, which shows the date of the generic name and its synonyma. When any new synonyma have occurred to me sinee the printing of that List, I have added the name after that of the gemus.

Some alteration has been made in the disposition of the families. The observations of Messi's. Cuvier and Quoy, strengthenel by the anatomical researches into the nervous system of these Mollusea by M. Gervais, and the researches into the structure and disposition of the siliceons teeth on the tongne-membranes of Dr. Loven, have shown that the Trochide, Turbinide and Haliotide must be removed from the other Phytophagons Ctenobranchiata, and that the Patellee and allied families, and probably the Chitones, should be arranged with them.

The admirable researches of Dr. Loren on the metamorphosis of Molhisea and the structure of the tongue of these animals, bas shown the neeessity of several new families being estahlished; for though the charaeter furnished by the teeth on the tongue is diffieult to observe, espeeially in the smaller animals, yet the modifications which they assume must be eharacteristic of important differences in the habit and organization of the animal.

The name of the author from whom the figure of the animal has been copied, precedes the reference to the plate in this work where the speeies is figured.

Class I. GASTEROPODA.

\section{Subclass I. CTENOBRANCHIATA.}

Order I. Pectinibranchiata.

\section{Suborder I. Zoophaga.}

Fam. 1. Strombidæ.

2. Aporrhaidæ.

3. Muricida.

4. Buccinidae.

5. Trichotropidæ.
Fam. 6. Conille.

7. Volutidx.

8. Cyplixadie.

9. Lamellariadæ. 
Suborder II. Phytophaga.

Sect. 1. Podophthalma.

lim. 10. Ampullariadie.

Sect. 2. Edriophthalma.
Fam. 19. Calyptradx.

20. Phorida.

21. Vanicorida.

E. 2.2. Valvaticle.

1. 23. Scalaridie. Sect. 3. Iniophthalma.

A. 24. Caeridax.

25. 'Trumeatellidie.

26. Prramidellidix.

27. Nitconide.

13. 2ล. Rissoellidie.

Order II. Scctibraxchiata.

Sect. 1. Podophthalma.

Fan. A. I. Turbinidae.

2. Liotialæe.

3. Trochida.

4. Stomatellidis.

5. Haliotidar.

B. 6. Neritinidac.
Sect. 2. Edriophthalma.

Fam. A. T. Fissurellicle.

8. Dentaliadie.

B. 9. Tecturidx.

10. Lepetidx.

11. Gadiniadic.

C. 12. Patellidac.

13. Chitonidlac.

\section{Subclass II. HETEROHRANCHIATA.}

Order III. Pleveromancinata.

Fam. A. 1. Philinide.

2. Bnllinadax.

3. Bnlliclas.

4. Amplnstridae.

B. 5. Aplysiadx.
Fan. C. 6. Lophocerciux.

7. P'leurobranchidx.

1). 8. Pterotracheidx.

9. Atlantislie.

10. Inuthinidlo.

\section{Order IV. Gymantanchitata.}

Fam. A. 1. Dorididix.

2. Tritoniads.

3. Glanciula.

4. Phyllidiadax.

5. Diphyllidiadx.
Vam. B. 6. Pluyllimhoidx.

7. Placoloranchidx.

8. Limapontiadie.

9. Sagitadac.

\section{Order V. PneumomRanchiata.}

adelofuncum ena $h \| /$ smborder I. INOPERCUIATA.

Fam. A. 1. Arionidac.

2. Helicidae.

3. Veroniecllidax.

4. Onchidiada:.
Fam. B. 5. Amriculida.

(i. Lymueadre.

C. 7. Siphonariade. 8 andutrites 



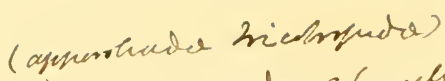

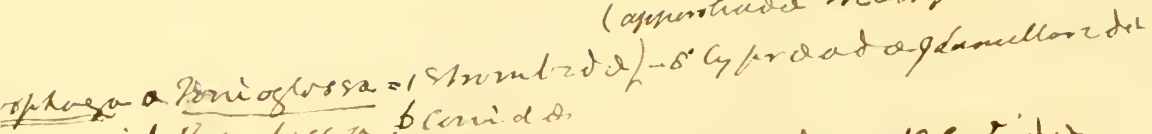

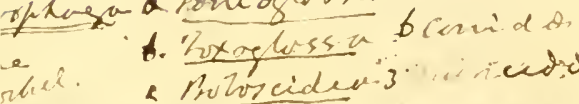

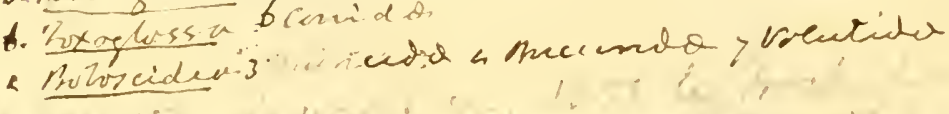

$-i l$

$\therefore l_{2} C-1$

- lis recendent menlle soge eqfended

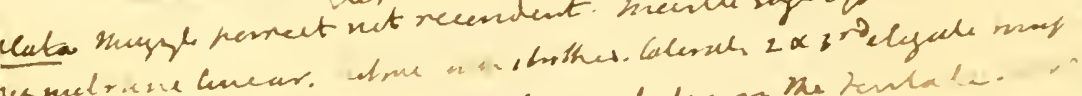

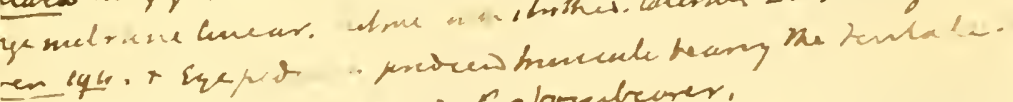

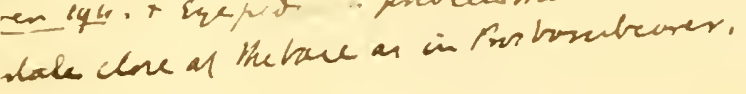




\section{Class I. GASTEROPODA.}

Adult walking on an expanded foot.

Subclass 1. Gill comb-like, formed of one or two series of lamella under the mantle on the back of the neck, or rarely round its edge. Adult and larva shell-bearing, larva furnished with deciduous cephalic fins. Unisexual, or hernuphrodite and self-impregnating. Ctenobranchiata, Gray, 1840. Prosobranchiata,

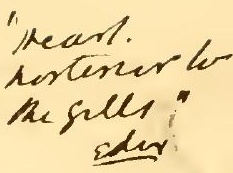
M. Edw. 1846.

\section{Order I. Pectininanchiata, Cuvier.}

Gill comb-like, formed of one or (rarely of ) two longitudinal series of laminæ on the left side of the mantle orer the back of the neck. Animal unisexual. Shell spiral.

\section{Suborder I. Zoophaga.}

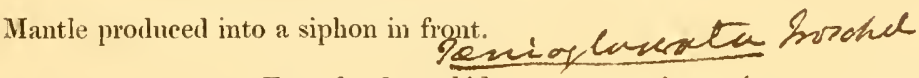

Fam. 1. Strombida.

alataxhares

Teeth, central 1, lateral 3.3, uncinated. Muzzle produced. Eyes pedicelled. Operculum small, claw-like, or wanting.

* Tentacle on middle of eye-pedicel. Operculum claw-like.

1. Strombus, Gray, Gen. Moll. n. 1=Pyramea et Lambis, Mus. Berl. 18$] 8$.

1. S. gigas, Kiener, t. 80. f. 2 ; Forskael, t.81.f. 4 ; Lamk.t. 83.f. 1.

2. S. Pugilis, Bowdich, t. 81 , f. 3.

3. S, Auris-Diana, Quoy, t.2. f. 1.

4. S. Canarium (var. Isabella), Quoy, t. 2. f. 4 б’, 3 f ; Eydoux, t. 81 a.f. 1.

5. S. gibberulus, Quoy, t. 81. f. 1.

6. S. Papuilio, Quoy, t. 8l. f. 2.

7. S. lentiginosus, Quoy, t. 81. f. 5.

8. S. varialilis, Quoy, t. 2. f. 2.

9. S. Luhuanus, Quoy, t. 2. f. 6.

10. S. plicatus, Quoy, t. 2. f. 5.

11. S. vittatus, Adams, t. 84 a. f. 5 .

2. Pterrocera, n. 2.

1. P. truncata, Kiener, t. 79 ; Freycinet, t. 70 . f. 5.

2. P. Lambis, Quoy, t. 1. f. $2 \delta$, f. 1 \%. Teeth of sexes different according to Quoy, Ast. t. 49 , f. 20 \% . t. 50 . f. 8 万'.

3. Fusus, n. 3.

$$
\text { ien opper zit. }
$$

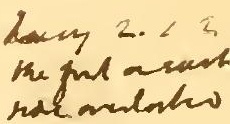

1. F. clavus. Rostellaria eurvirostris, Kiener, t. 80.

2. F. subulatus, Adams, MSS. t. $84 a$. f. 6 .

$$
\text { ** Tentacle none? Operculum Llarlike.. }
$$

4. Seraphys, n. $4=$ Terebrina, Rafinesque. Oper. none.

1. S. Terebellum (Terebellum subulatum), Adams, MSS. t. $84 a$. f. 3, t. 82 . f. 3. 
alalatherean Finn. 2. Aporrhuide.

'Tectl, eentral 1, lateral $3: 3$, uncinate. lyes sessile at base of tentade. Varix single, premanent, marginal.

en t 4623 - 1. АровRHAis, 11. 33. Oper. orate. a hamanala

1. A. Pes-peleeani, Miiller, t. 91. f. 3; Chiuje, t. 91. f. 4; Desk.t.91.

f. 4; Joannis, t. 91. f. 1 ; Johnston, .11SS. thel f f'?

2. ? Strethiolaria. Oper. ?

1. S. reruis. S. (remulata, Quoy, t. 5. f.3; hiener, t.9). f. 6.

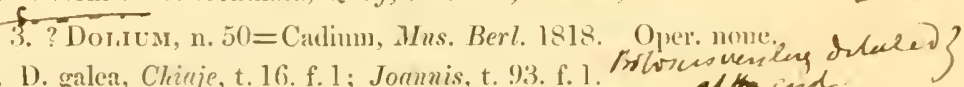

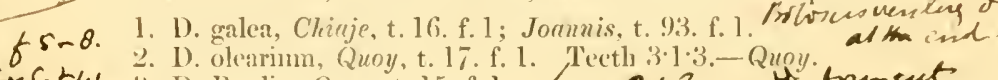

3. D. Perdix, Quoy, t. 15. f. 1

3.1 .3 -mati froment

* rotrociden

Fan. 3. Muricille. Muricea, Lovin.

Tectl, central l large, lateral $1 \cdot 1$, versatile. Muzzle retractils. \&. Operenlum ammlar. Siplion of mantle produced in front.

stonsmu.* Operculum ovate, anmular;"nueleus subarical within the upeir. avete anmiton 1. APOLLON, n. $5=$ Gyrinium, Mus. Berl. 1818.

elande cestere T. A. vexillum. Ranella v., Quny, t. 265. f. 1. Oper. like Triton.

2. 1. foliatum, Adems, t. $84 a$. f. 1 .

3. A. bufonim, Quoy, t. 4. f. 2.

4. A. graniferum, Quoy, t: 4. f. 4.

5. ג. lencostomum. 'T'iton 1., Quoy, t. 85. f. 1 ; Desh.t.116. f. 2.

6. A. retienlare. Ranella gigantea, Kiener, t. \$6. f. 1.

7. 1. erelatum. Oper. like Triton.

2. Triton, n. $6=\mathrm{Ne}^{\mathrm{p}} \mathrm{ptunea,} \mathrm{Mus.} \mathrm{Berl.} 1818$.

1. 'T. nodifermu, Chinje, t. 3. f. 3 б; Kiener, t. \$5. f.5; Bowd. t..3. f. 4 ?

2. 'T. olearimm. 'T. succinctum, Chiaje, t. 3. f. 2); Quny, t. 263. f..3.

3. 'l. cutaceum, Chiuje, t. 3. f. 1 ' .

4. 'T. tuberosum, Quoy, t. 4. f. 1-6.

5. 'T. pileare, Quoy, t. 4. f. 38 .

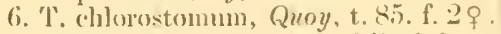

7. 'I'. cancellatum, Quoy. t. 2(i5. f. 2.

8. 'T'. rubecula? Swainson, t. 85. f.3.

9. T. Spengleri, Quoy, t. 4. f. 5.

3. Prersoxa, n.7 = Distortrix, Mus.Berl.1s]8. Oper. small. broal.

1. 1'. anus. Triton a., Qnoy, t. 85. f. 4. hem 540 of

4. Murex, 11.10.

with

mo $1.2 \mathrm{mg}=3 \hat{\jmath}$. M. inflatus, Kiener, t. 84.

2. Ml. temusplimus, Quoy, t. 6. f. 2.

3. M. Bramlaris, Chirje, t. 6. f. $3 \delta^{7}$, t. $7.1 .1,3$.

4. M. erinacens, Chiuje, t. 84) f. (i.

5. M. palma-rosar, Quoy, t. 7. f. (i.

6. MI. zenlandicus, Qnoy, t. 7. f. 3.

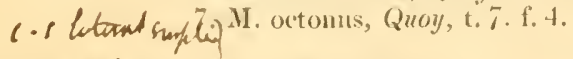

entsplo. he comutas. 
musph ferscel rentrach

s

$\mathrm{mm}$

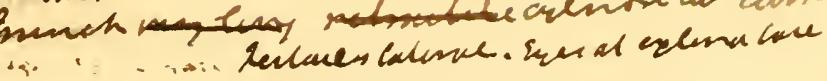

erestate concian

monther cass

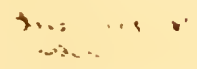
$\because$

$\therefore i$ is

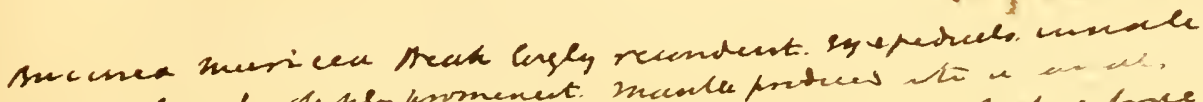

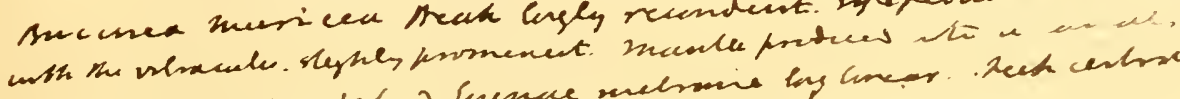

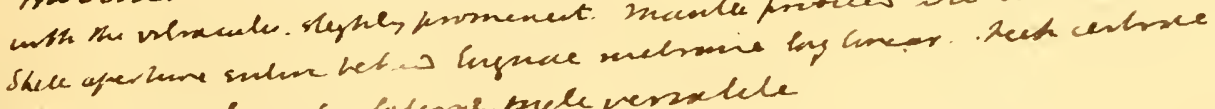

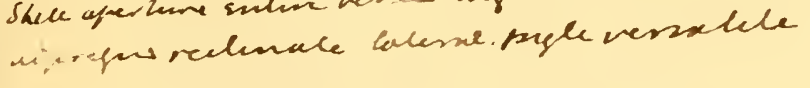




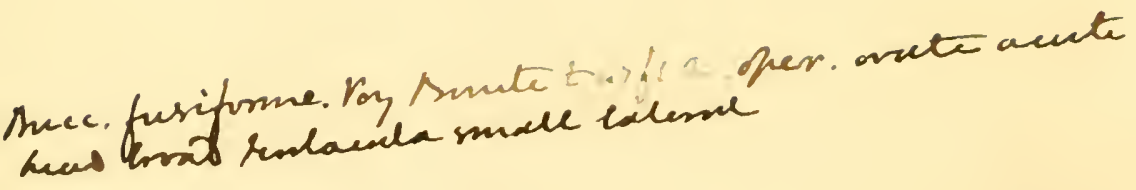

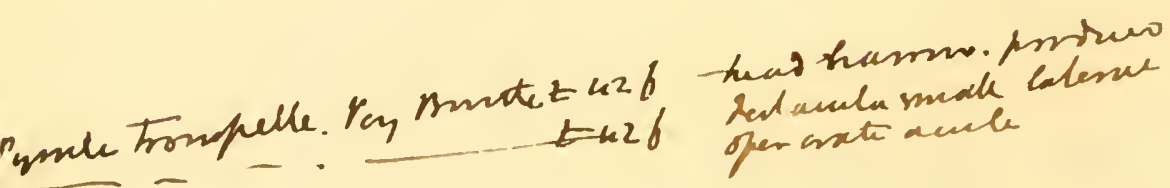
operate acuale 
8. M. Trunculus, Chiaje, t. 6. f. 1 ठ̊; Fab. Colum. t. 89. f. 7.

9. M. ricimuloides, Quoy, t. 7. f. 5.

5. T'Ropilon, n. 11.

1. T. patagonicus. Murex p., D'Orb. t. 89. f. 2.

2. T.? erassilabris. Purpura c., Eydoux, t. 87 a. f. 2 ; Murex labrosus, D'Orb. t. 89. f. 4.

** Operculum ovate, acute; nucleus apical. Varices of shell rudimentary or none.

\section{Pisania, n. 4=Polliana, Gray, Fig. Holl. $=\boldsymbol{s}$.}

1. P. striata. Buccinum maculosum, Joannis, t. 90. f. 3. Colum-

bella mercatoria, Chiaje, t. 98. f. 1, t. 98 . f. 8.

2. P. - - Bucc. civette, Eydoux, t. 87 a. f. 3.

3. P.? lineata. Fusus l., Quoy, t. 5. f. 5.

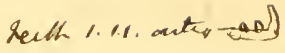

4. P. ignea, Bucc. flammulatum, Quoy, t.5. f. 2.

5. P. cincta. Buce. c., Quoy, t.5. f. 7.

6. P. violacea. Bucc. v., Quoy, t. 5. f. 8.

7. P. fasciolata. Bucc. f., Quoy, t. 5. f. 4.

8. P. - - n. s., Chiaje, t. 102. f. 20.

9. P. undosa. Bucc. u., Quoy, V. A. t. 30. f. 1-1.

10. P. vittata. Fusus v., Quoy, V. A. t. 34. f. 18, 19?

11. P. lincolata. Bucc. 1., Quoy, t. 5. f. 1 .

12. P. littorinoides. Bucc. litt., Reeve, B. $M$.

13. P. antarctica. Bucc. a., Reeve, B. M.

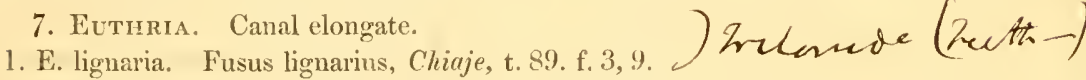

8. Enzrna, n. 9. Oper. ovate, acute, curved. E. turbinella. Purp. t., Sow.

9. Colus, n. 19=Fusinus, Rafin. Animal rerl. Opcreulum orate, acute.

1. C. multicarinatus. Fusus m., Kiener, t. 90. f. 2 .

2. C. anstralis. Fusus a., Quoy, t.9. f. 3.

3. C. —? Chiaje, t. 9. f. 2 .

4. C. raphanus, Quoy, t. 9. f. 1.

$42 \times 8.3415$ C. clilatatus, Quoy, t. 9. f. 5.

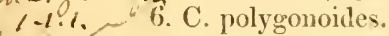

10. Cassidulus, n. $21=$ Mancimella, Mius. Berl. 1818.

1. C. Melongena, Kiener, t. 88. f. 1.

2. C. galeodes, Quoy, t. 266. f. 1.

3. C. Cochlidium. Pyrula C., Quny, t. 266. f. 2.

4. C. Vespertilio.

5. C. Colosseus.

Luth 1.11. 2unte34/15.

henet hare

Laven $\angle 5 / 61.1$

11. Latirus, 11. 25. Animal led.

1. L. lineatus. Turbinella 1., Quoy, t. 8. f. 3.

2. L. polygonus. Turbinella p., Quoy, t. 8. f. 7.

3. L. nussatellus, Turbinellus n., Quoy, t. 8. f. 1.

12. Fasciolaria, n. $26=$ Ieraneæ, Rafin.

1. F.tulipa, Kiener, t.86. f. 2; Lamk. t. 87.

1.1.1 2. F. filamentosa, Quoy, t.8. f. 5 .

3 F. Tarentina, Chiaje, t. 8. f. 4 ; Joannis, t. 89. f. 


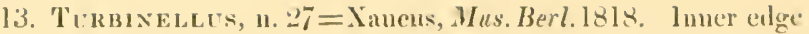
of opere straight. 'T, ovoidens.

14. Crxononta, n. $2 S=$ Tasmu, Mus. Berl. 1818. Animal olive. Operenlar imner ellge curved.

1. C. comigera. Turb. c., Quoy, t. 8. f. :3, copied Kiener.

2. C. ceraniea. Thul). c., Quoy, t. 93. f. 3.

3. C. pugillaris.

15. LAGENA, 11. 2Y.

/-ノ. I - 1. L. lustica. 'Turbinellus r., Quoy, t. 8. f. 6.

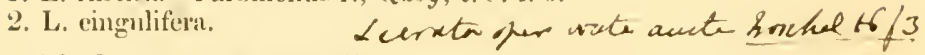

16. Chirsodone's, 11. :39.

$t_{5} f^{1-2 \cdot 3--~ 1 . ~ C . ~ a n t i q g u m s . ~ M u r e x ~ a ., ~ M u ̈ l l e r, ~ t . ~ 92 . ~ f . ~ 1, ~ t . ~ 8 \% . ~ f . ~ 8 . ~}$

17. Clavelda, n. 37=Cyrtulus, 11, 38. P'ollia, sp. Gray. Columbella, sp. Duclos. C. distorta. Pollia distorta, Gray. Oper, thick. C. serotina. Cyrtulus s., Hinds. Oper.-—?

\section{Cochlidiun.}

*** Operculum none.

Head elongate conical, like a snout, with a very long, exsertile proboseis. 'Tentaenla very small, on the side of the month. Eyes small, on onter side of their hase. Male organ large. Foot ovate,

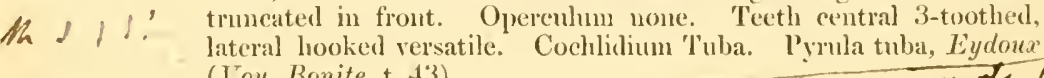

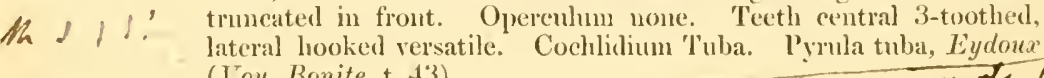

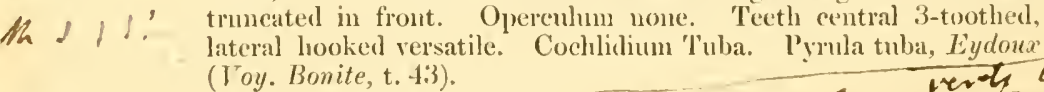
19. Srcotrpus, n. 20. Rakh 3.1-3. Trhorcir

1. S. fieus. Ficula levigata, Adams, MISS. t. S6. f. 2, corrected Luen? t. 261 . f. 4.

2. S. reticulata. Ficula 1., Adams, t. 261. f. 5 .

20. Cancellaria, n. 30.

1. C. tulber(ulata, D'Orb. t. 89. f. 5, altered Kiener, t. 91. f. 5.

2. C.? (Admete) crispa, Moller, t. 125. f. l.

***** Operculum semi-onute; aucleus small, in the middle of the straight inner edge, like Bircardica.

21. PUSionela , 11. 40. hay M.zS COSO

1. P. Nifat. Fusis N., Lamk.

2. P. buccinatus. liusus b., Lamk.

3. P. aculciformis, Fusus a., Lamk.

***** Operculum oralc, blunt; nuclens elongate, occupying great part of outer or hinder side, like l'urpura.

22. RАPANA, 11. 23.

Whe 1. 1. T.

slusin la lant rearfuce
1. R. Bezo:rr. Huc, B., Linu.

2. R. Rapa. Bulla Rapa.

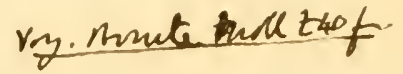

2:3. Chorus, 11. :34.

I. C. giganteus. Mhonoceros g., Jiesson.

24. $\operatorname{Cum}$ A, 11. 24.

I. C. sulcata, Swuius. 


Fam. 4. Buccinida.

Tecth like Muricida. Siphon of mantle recurverl.

a. Operculum ovate or oblong, nucleus on middle of inner or frout edge.

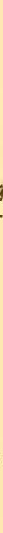

5. HARPA, n. 52=Harparia, Rafin. 1815=Harpalis, Mus. Berl. 1818.

1. H. ventricosa, Quoy, t. 22. f. 1, 2; Lister, t. 95. f. 3.

2. H. minor, Quoy, t. 23. f. 1, 2.

c. Operculum oblong; nucleus elongate, occupying the long outer edge. Purpurina.

6. Columbella, n. 53=Peristera, Rafin. 1815=Pterygia, Mus. Berl. 1818.

1. C. rustica. Vohuta r., Chiaje, t. 11. f. 6, 7, t. 98. f. 2 ; Joannis, t. 98 . f. 4,5 .

2. C. criloraria. Buccinum c., Quoy, t. 24. f. 6. Oper. like Purpura.

3. C. mendicaria, Quoy, t. 98. f. 7. "Oper. ovate, acute." ofay a wa h

4. C. Paytensis. C. Paytalida, Kiener, t. 98. f. 6.

5. C. pardalina, Quoy, t. 98. f. 3. "Oper. ovate, acute."-nulen ofriane

6. C. labrosa, Eydoux, t. 81 a. f. 4. Oper. oblong, nucleus lateral.

7. C.? lanceolata, Eydoux, t. 81 a. f. 2.

8. C. - ? Barnet, Adanson, t. 24. f. 10.

9. C. Scrtulariarum. Buccinum S., D'Orb. t. 99. f. 5.

7. Purpura, n. 55.

1. P. hæmastoma, D'Orb. t. 106. f. 1; Joannis, t. 97 . f. 7 .

2. P. patula, Kiener, t. 95 . f. 2.

3. P. rugosa, Quoy, t. 96 . f. 7.

4. P. Thiarella, Quoy, t. 96. f. 8.

5. P. hippocastanum, Quoy, t. 96. f. 9.

6. P. mancinella, Quoy, t. 96 . f. 12 .

7. P. chocolatum, D'Orb. t. 97. f. 6.

8. P. sertum, Quoy, t. 97. f. 3.

9. P. armigera, Quoy, t. 14. f. 4.

10. P. textilosa, Quoy, t. 14. f. 6. 
11. P. coronata, Adanson, t. 14. f. 5.

12. P. haustrum, Quoy, t. 13. f. 2, 6.

13. P. kiosifuiformis, Eyloux, t. \&7 u. f. 6 .

14. P. undiati, Eydloux, t. 87 a. f. I.

15. P. I Iarpa, Eydoux, t. si a. f. 10.

16. P. cassidiformis, Eydoux, t. \&7 a. f. \&.

17. P. sertularia, D* Orb. t. 99. f. 5.

18. P. - Pourpre de Chusan, Eydoux, t. s7 a. f. 6.

19. P. echinulata, Quoy, t. 266. f. 4.

20. P. Quoyii. Buc. testulinem, var. Quoy, t. 13. f. 7.

21. P. IIelena, Quoy, t. 96. f. I.

2.2. P. tubereulata, Fydoux, t. 87 a. f. 4 .

23. P. neritoidea, Quoy, t.96. f. 10. P. matheporarum, Quoy, t. 26iti. f. 3 .

24. P. hystrix, Quoy, t. 96. f. 4. Pourpre ouverte, Eydoux, t. 87 «. f. 7 .

25. P. —, Quoy, t. 96. f. 5. Animal eut open.

8. Concholepas, n. 56* = Conchuhs, Rafiu. 1815. Leelt

i. C. peruviama, Lesson, t. 13. f. 1, 3; Kiener, t. 95. f. 1

9. Acanthiza, n. 57.

1. A. striata. Monoceros striatum, Eydoux, t. si a. f. !.

2. A. unicornis. Mou, crassilabrum, D'Orb. t.97. f. :2.

10. Sistrum, n. 60 .

1. S. ricinus. Ricinula arachmoides, Quoy, t. 96. f. 2.

2. S. dactyloides. Ric. digitata, Quoy, t. 96. f. 3.

3. S. nodim. Ric. morus, Quoy, t. ?) f. f. 6.

4. S. horridun. Ric. h., Quoy, t.96. f.11; Kiener, t.97. f.5; Freycinet, t. 13. f. 4.

11. Campulotus, n. 61 .

1. C. antiquus. Magillus a., Rïppell, t. 24. f. 5, t.

d. Operculum ovate; nucleus small, near outer front pdye. Buicinina.

12. Buccrusa, n. 62=IIalia, Macyilliv.

en $t 5(4-5$. 1. B. undatun, Cuvier, t. 94. f. 1; Miiller, t. 9. f. 1, t. 9.2. f. 4.

13. Planaxis, 11. 58.

I. P. pyramidalis, P. suleatus, Quoy, t. 2-1. f. 6.

2. P. nigra, Quoy, t. 24. 1.3.

14. QUOYIA, 12. 59. ger foret ady ended on fond.

1. Q. decollata. Planaxis d., Quoy, t. 24. f. 1.

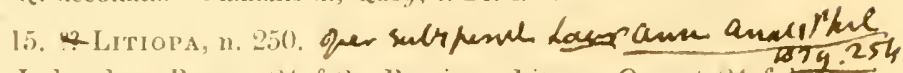

1. L. bombyx, liang, t. 24. f. 2. Bur(ium Litupa, Qnoy, t. 24. f. t.

2. I. melastoma, Eiycloux, t. 107, f, 3. Oper., spiral: opereular mantle with tentactes'
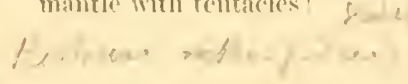


f. Operculum ovate lanceolate; nucleus apical, outer edye often ser-

rated. Nassina.

16. Latrunculus, n. 63. Oper. ovate, acute, erlge entire.

1. L. areolatus, Eburna areolata, Kiener, t. 93. f. 2.

2. L. spiratus. Ebuma s., Eydoux, t. 85 a. f. 4.

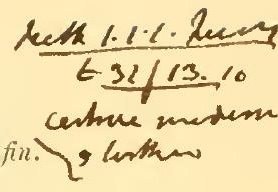

17. Acus, n. 65. Oper. ovate, nucleus apical=Terebraria, Rafin

1815=Vertagus, Mus. Berl. 1818. Fif. . elyale .m

1. A. maculatus, Terebra m., Eydoux, t. 85 a. f. 8; Kiener, t. 99.

f. 1 .

2. A. subulatus. Terebra s., Quoy, t. 99. f. 3.

3. A. climicliatus. Terebra d., Quoy, t. 99. f. 4.

4. A. strigilatus. Terebra s., Eydoux, t. 85 a. f. 9

5. A. ? anatomy, Quoy, t. .99. f.

18. Dorsanum, n. 66 .

1. D. politum. Buccirum p., Lamk. Miran, Adanson, t. 24. f. 12, 13.

19. Bullia, n. $67=$ Bulliana, Gray, Fig. Moll. 1812.

1. B. lævigata. Buccinum lævissimum, Freycinet, t. 20. f.3; Qnoy, Mybos t. 20. f. 2 .

2. B. ligitalis. Bucc. achatinum, Quoy, t. 20. f. 1 .

3. B. Cochlidium. Buce. C., D'Orb. t. 98 . f. 9.

4. B. globosa. Bucc. gl., D’Orb. t. 98, f. 10.

20. Nassa, n. 68=Nassaria, Mus. Berl. 1818.

1. N. coronata. Buccinum c., Quoy, t. 21. f. 2.

2. N. arcularia. Buccinum a., Forsk. t. 98. f. 14; Quoy, t. 21.f. 1 ;

Lister, t. 102. f. 11; Chiaje, t. 21. f. 9, ]0.

3. N. olivacea. Buccinum o., Quoy, t. 21. f. 6 ; Eydoux, t. 85 a.

f. 2 , var. f. 6 .

4. N. gemmulata. Buce. g., Eydoux, t. 85 a.f. 7.

5. N. cremulata. Buce. c., Eydoux, t. 85 a. f. 3.

6. N. xanthostoma. Bucc. x., Eydoux, t. 85 a. f. 5.

7. N. Isabella. Bucc. I., D'Orb, t. 98, f. 12.

8. N. dentifera. Buce. d., D'Orb. t. 98. f. 11.

9. N. - Buce. ripariun, Chiaje, t. 97. f. 1.

10. N. Thersites. Bucc. Th., Quoy, t. 2l.f. 3.

11. N. pamperata. Bucc. p., Quoy, t. 2l. f. 4.

12. N. fasciata. Bucc. f., Quoy, t. 21. f. 5.

13. N. globosa. Bucc. g., Quoy, t. 2l. f.7.

14. N. reticulata? Buce. r.? Quoy, t. 21. f. 8.

15. N. gibba. Buccinum mutabile, Lamk.; Lister, t. 102. f. 11;

Chiaje, t. 21.f. 9, 10; Forskael, t. 21. f. 11.

16. N. cornicula. Bucc. c., Chiaje, t. 24. f. 11.

17. N. ascanias. Bucc. pygmeum, Chicie, t. 21. f. 12.

18. N. - F. Fub. 85 r. f. 1, 2, 3, 5, 6, 7 .

19. N. (Cyclope) neritea. Bucc. 11., Chiaje, t. 24. f. §.

20. N.? Adanson, t. 102. f. 13.

21. Northia, n. 70.

1. N. pristis? Adams, t. $84 a$. f. 4.

22. Phos, n. 72. Oper. ovate, nucleus apjeal, P. senticosus

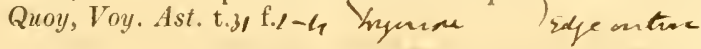


23. Cominella. Oper. ovate acute, outer edge smooth.

1. C. testulinea. Buce. t., Quoy, t. 14. f. 1, 3.

2. C. maculosa. Purpura m., Lamk.; Reeve, f. 85. 、

3. C. plurianmulata. Bnce. p., Reeve, f. 38.

4. C. ligatit. Buec. l., Lamk.; Reeve, f. 22.

5. C. lagenaria. Bucc. 1., Lamk.; Reeve, f. 33, 34.

6. C. limbosa. Bnce. I., Lrmmk.; Reeve, f. 35.

7. C. lineolata. Buce. l., Lamk.; Reeve, f. 36.

8. C. acutinorlosa. Bucc. a., Reeve, Conch. t. 4. f. 21.

9. C. costata. Buce. c., Quoy, t. 102. f. 9.

g. Shell plaited in front. Olivina.

24. Strepiona, n. 74=Olivaria, Rafin. 1815=Porplyyia, Mus. Berl. 1818. Frontal lobes small, aeute; opereulum none.

1. S. sanguinolenta. Oliva s., Quoy, t. 18. f. 1.

2. S. textilina. Oliva t., Quoy, t. 18. f. 2.

monel 1.143 . S. elegans. Oliva e., Quoy, t. 18. f. 3.
4. S. ervthrostoma. Oliva e., Quoy, t. 18. f. 4.

growe 1.143 . S. elegans. Oliva e., Quoy, t. 18. f. 3.
4. S. ervthrostoma. Oliva e., Quoy, t. 18. f. 4.

5. S. Manura. Oliva M., Quoy, t. 18. f. 5, t. 102. f. 5.

6. S. senegalensis. Oliva s., Eydoux, t. $83 a$. f. 1 .

7. S. Ispidula. Oliva I., Duclos, t. 101. f. 2, 3.

8. S. - - Oliva, Duclos? t. 100. f. 3.

25. Olivella, n. 75. Frontal lobes small, aente; operculum distinct, half-orate; muclens subapical.

1. O. volutella. Oliva v., Eydoux, t. $83 a$. f. 2 .

2. O. colunellaris. Oliva c., Eydoux, t. 83 a. f. 3.

3. O. Pnelchana. Oliva P., D'Orb. t. 102. f. 1; Duclos, t.101.f.l.

4. O. Tehuclehana. Oliva T., D’Orb. t. 102. f. 2 ; Duclos, t. 101. f. 4.

26. Scapinta, n.76. Frontal lobes very large, rounded; operculum distinct, half-ovate; nucleus subapical.

1. S. auricularia. Oliva a., D'Orb.t. 102. f. $6 ;$ Duclos, t. 100. f. 1 .

27. Araronia, n. 7\%. Frontal lobes moderate, acute; operelllum distinct.

1. A. hiatula. Oliva h., Duclos, t. 101. f. 4, 6, 7.

2․ Axcilla, n. 78. Oper. small, orate, acute. or hume

1. A. australis. Ancillaria a., Quoy, t. 19. f. 5. - ches namom

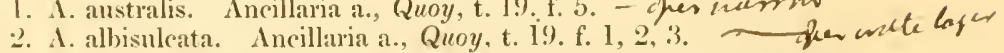

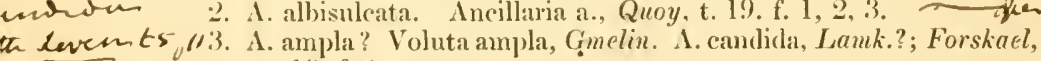

ne monsinthen t. 19 . f. 4 .

warkerbue 4. A. - - ? Adams, t. 86 a. f. 1.

5. A. mauritiuna. Ancillaria volutella, Kiener, t. 105. f. 1. ofen nuve

Pam. 5. Trichotropida.

'Tooth central, single, lateral 3:3, converging. Operculun homy; nucleus lateral, like Buccinida.

1. 'Tricıotropis, n. 64.

1. 'T. bica'uata, Sowerby, t. 24. f. 7 .

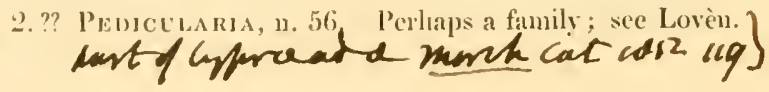


2

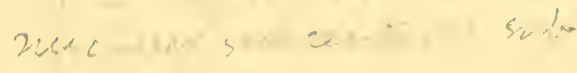

$-\alpha$ 1). 2

ret! ! : $\lim _{1} \hat{A}, \ldots+\ldots, \ldots, \ldots$

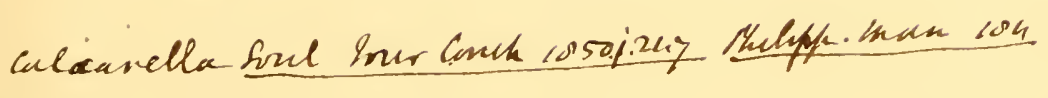
c.ppurans 




$$
\cdots+1 ., \quad 2 .
$$

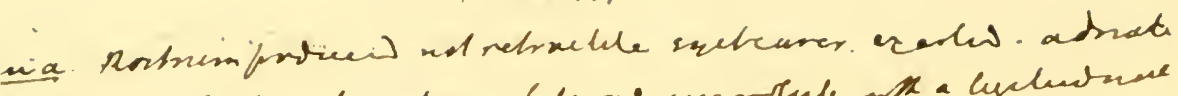

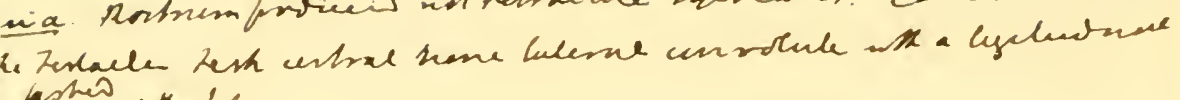
athen an the

$$
\text { (ke 35/5) }
$$

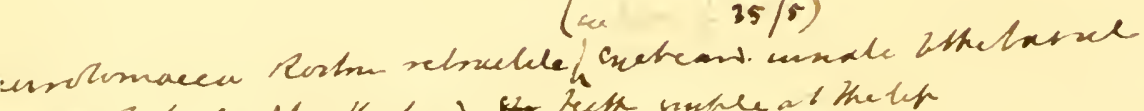

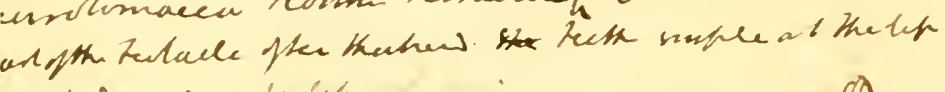
nuspormes anle is

enovifer. toy mute tunj8.6 ofer

(Q)

L. $\infty$ 


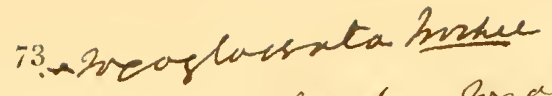

Fan. 6. Conida. = Revolsasulua ho aglosen

Teeth in pairs, elongate, subulate or hastate.

a. Operculum ovate, acute, nucleus apical. nacl, nme

en t $_{\text {S }} / 4$ 1. Covus, n. $28=$ Comulus, Rafin. Oper, thick, flat above, ovate,

linear, apex acute, front or inner side straight.

1. C. bandanus, Lesson, t. 11. f. 8, t. 10. f. 7 ; Quoy, t. 83. f. 2.

2. C. mediterranens, Philippi, t. 83. f. 4.

3. C. acuminatus, Ehrenb. t. 83. f. 3.

4. C. - Eydoux, t. 81 a. f. 6.

5. C. pulicarius, Quoy, t. 10. f. 8.

6. C. marmoreus, Quoy, t. 10. f. 5.

7. C. hebræus, Quoy, t. 10. f. 4.

8. C. figulinus, Quoy, t. 10. f. 3 .

9. C. Miles, Quoy, t. 10. f. 2.

10. C. betulinus, Quoy, t. 12. f. 1.

11. C. lividus, Quoy, t. 12. f. 3.

12. C. tæniatus, Quoy, t. 12. f. 5.

13. C. arenatus, Quoy, t. 12. f. 8, 9.

15. C. raphanus, Qmoye, at. 11. f. 3.

16. C. ? rusticus, Chiaje, t. 11. f. 4 .

17. C. sanguinolentus, Quoy, t. 11. f. 5 .

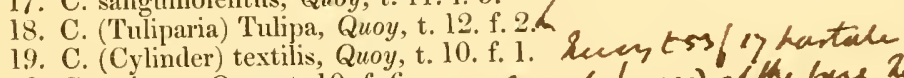

20. C. striatus, Quoy, t. 10. f. 6.

5. 1 . 1 .

2. Pleurotoma. Turris, n. 12. Oper. ovate-lanceolate, tiat,

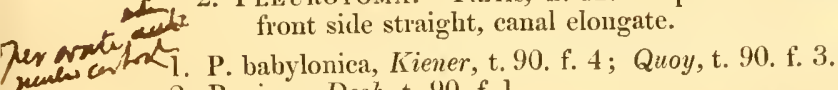

2. P. virgo, Desh. t. 90. f. 1.

3. P. oxytropis, Lamk. -

kay Mnate t4k69-10 dier

3. Drillia, Gray=Clavatula, n. 13, pars. Oper. like Pleurotoma, canal short, eyes on upper part of tentacula.

1. D. —, Adams, t. 84 a. f. 2.

2. D. ? echinata. Pleurotoma e., Quoy, t. 91. f. 4., arofermate andere

b. Oper. semiovate annular, nucleus on the centre of the straight iblylonico front edge.

4. Clavatula, n. 13, pars. Shell canal short. 1. C. bimarginata. Plenrot. b., Lamk. 2. C. imperialis. Pleurot. i., Lamk.

5. Tomella, Swains. 1840. Canal elongate, immer lip callous near suture. 'T. lineata, Swains. Pleurotoma l., Lamk.

c. Operculum none.

6. Defrancia, n. 16.

1. D. multilineata. Plcurotoma m., Philippi, t. 90. f. 2, t. 105. f. 5 .

7. Daphelela, Hinds, n. 13, pars, not Baird. D. marmorata. Oper. - ? 


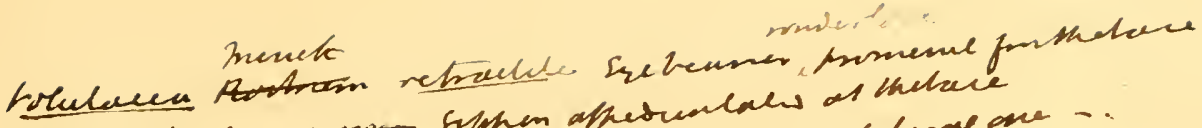

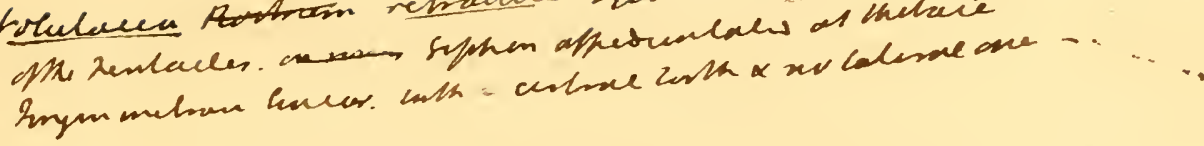

$$
\text { . } \therefore ; \quad \cdots \cdots+\cdots
$$

bocuta oper. onete

rimesica

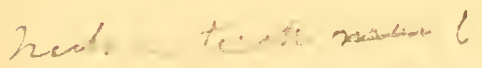



10. Persicula, 11. 101=Marginella, Mus. Berl. 1818 .

1. P. carneola? Oliva c., Chiaje, t. 102. f. 3.

Fam. 8. Cypraada.

Teeth central single, lateral $3 \cdot 3$, converging. ofuenulen nowe

1. CYPR玉a, 11. 103 .

$$
\text { cisnde recuers }
$$

1. C. stercoraria. Maget, Adanson, t. 35. f. 4.

$4.1-4-2$. C. Tigris, Quoy, t. 32. f. 1 9 , t. 35 . f. 6 б; Freycinet, t. 30. f. $1,2$.

3. C. Tigrina, Ehrenbery, t. 31. f. 3; Forskael, t. 35. f. 5.

th $2 \times 8-$ 4. C. mamitiana, Quoy, t. 31. f. 1; Nelson, t. 36. f. 5 . Young. Pe8 f $3 \cdots 4-4$.$) ribolus, Adanson, t. 35. f. 2$.

5. C. Isabella, Quoy, t. 36. f. 3.

6. C. Talpa, Quoy, t. 31. f. 2.

7. C. arabica, Quoy, t. 33. f. 6, t. 34. f. 1; Eydoux, t. $83 a$. f. 5.

8. C. variolaria, Quoy, t. 3t. f. 3.

9. C. vitellus, Quoy, t. 34. f. 5.

10. C. rufa, Chiaje, t. 35. f. 1-3.

11. C. crihraria, Quoy, t. 34. f. 2 .

12. C. anmulus, Quoy, t. 34. f. 6.

13. C. Moneta, Quoy, t. 34. f. 9.

14. C. Caput Serpentis, Quoy, t. 33. f. 4.

15. C. olivacea, Quoy, t. 34. f. 8 .

16. C. Caurica, Adams, t. 261.f. 1.

17. C. erosa, Quoy, t. 33. f. 1, 2; Adams, t. 261 . f. 2.

18. C. nucleus, Quoy, t. 34. f. 4.

2. Trivia, n. $104=$ Coccinella, Searles Wood, not Linn.

1. T. europæa, Mrs. Johnston, t. 36. f. 1. C. pediculns, Guerin,

t. 36. f. 4. C. norvegica, Sars, t. 36. f. 6.

2. T. australis, Quoy, t. 3 \%. f. 7 .

3. ErAto, n. 107.

1. E. Cypreola? Oliva carneola, Chicie, t. 102. f. 7.

4. Auphićeras, n. 10 = Cyphona, Hus. Berl. 1818.

1. A. ovum. Ovula ovum, Freycinet, t. 29. f. l, 2; Quoy, t. 30. f. 3, copied; Kiener, t. 105. f. 4 .

2. A. torile. Ovula angulosa, Quoy, t. 33. f. 3, 5 .

3. A. dentatum. Ovilum d, Allams, t. 86 u. f. 6 , t. 261 . f. 3.

4. A. verrucosum. Oxulum v., Quoy, t. 36 . f. 2 ; Adams, t. 86 a. f. 5 .

5. A. volva, Adams, t. 86 a. f. 3.

6. A. adriaticum. Ovula a., Philippi, t. 105. f. 7 .

7. A. spelta. Ovula s., Philippi, t. 105. f. 6.

\section{Fam. 9. Lamellariada.}

Teeth central single, lateral 3.3 (or 1.1 very large), converging; mantle covering the shell not retractile; muzzle retractile.

1. Lameduaria, n. $109=$ Sigaretia, Rafin. 1815. Teeth, central 1, lateval 1.1, large, versatile. Loven, t. 4. f. 15; Quoy, I. A. t. 6.t*. f. 8 .

1. L. perspicua. Iamellaria tentaculata, Montay. t. 103. f.11. Si-

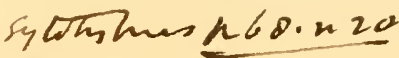


garetus morelli, Chinje, t. l(13. f. 4. S. perspicuus, Philippi, Cantraine, t. 105. f. "2, t. 105. f. 3.

2. L. Adansonii. Sigaretus A., Chiuje, t. 10:3. f. 9.

3. L. neritoidea. Sigaretus n., Chiaje, t. 103. f. '2.

4. L. - n. Sigaret convex, Blainv. t. 103. f. 5. These may be all one species.

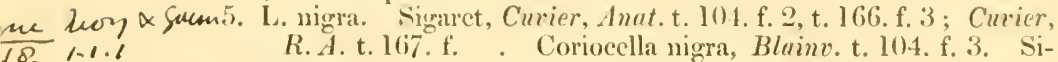
garetus Tonganus, Quoy, t. 10-4. f. 4.

2. Marsenisa. Teeth, eentral 1 , lateral $3: 3$, hooked, equal. II. prodita. Latmellinia prodita, Lovèn.

\section{Suborder II. Phrtupliaga.}

Mantle not (or rarely) produced into a siphon.

\section{Sect. 1. Porlophthalma.}

Eyes on perluncles, separate from the tentacula. Male organ on edlge of mantle. Gill-carity with a short fringed gill and a large pulmonary bag on each side. central.-Quoy, I. A. t. 57. f89. Mantle with a more or less long siphon on left side; rectum not traversing the heart. Fluviatile.

\footnotetext{
1. AnPtLlaria, n. I66. Opereulum horny, with a slelly intemal coat. Respiratory siphon etwere. usteyphas

1. A. fasciata, Hardwicke, t. 47. f. 3.

C. A. Cehensis, Quoy, t. 47. f. 1, 2 \& 4.

2. Marisa, n. 167. Opereulum horny, siphon clongate. M. cor-

3. Powus, n. 168 . Operculum horny, siphon elongate.

1. P. zonatus. Ampullaria z., D'Orb. t. $47^{*}$. f. 2.

2. 1'. Rossii. Ampullaria R., D'Orb. t. $47^{*}$. f. I.

3. I'. Scalaria. Ampullaria S., D'Orb. t. 47**. f. 2.

4. P. camaliculatus. Ampullaria c., D'Orb. t. $47^{* *}$. f. 1 .

5. P. insularum. Ampullaria i., D०Orb. t. 117 . f. 1.

(i. 1'. - Guilding; Suains. t. 117. f. '2.

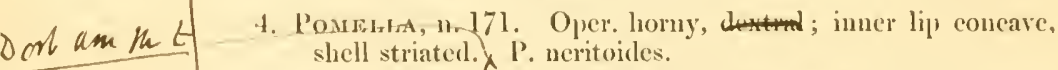
shell striated. $\times$ P. neritoides.

5. LAxistes, n. 169. Oper. horny, or with melens on left inargin. L. Bolteniana.

(i. Mela donus, n. 170. Oper. homy, smimed. M. olivacens.

7. Asotexe, n. 172. Opereulum loruy, with an interual shelly coat ; siphom not exposict.

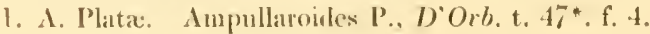




sect. 2. Edriophthalma.

Eyes sessile, or on very short tubercles on the outer side of the base of the tentacula. Male organ on right side of nape.

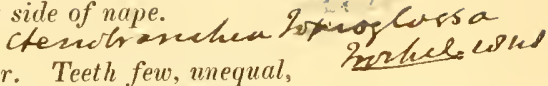

A. Shell free, spiral. Opercnlum annnlar. Teeth few, nnequal, 1 central and 3 lateral on each side, converging. Male organ at base of right tentacle, Lister, Cuvier. Gills three ranges of filaments. "Fluviatile.

Fam. 11. Viviparidee.

1. Viviparus, n. 251 = Viviparella, Rafin. Operculum horny, nucleus ncar the midlle of inmer side.

1. V. vulgaris. Helix viviparus, M̈̈iller, t. 124. f. 3 ?; Cuvier, t.124. f. 6 . Nerita viviparus, Sturm, t. 124. f. 9.

2. V. bengalensis, Hardwicke, t. 124. f. 2. India.

3. V. ampullariformis. Paludina a., Eydoux, t. 127 a. f. 5.

4. V. dlecisa, Haldm. t. 310 . f. 10.

2. Paludomus, n. 252 =Tanalia, n. 219 , is only a sublivision. Operculum homy, nuclets near the front of outer sile.

-1. P. conicus, Gray, P.Z.S. 1849.

2. P.? Paludine boueuse, Eydoux, t. 127 a. f. 4, edge of mantle festooned.

3. Brturin, n. 253. Operculum with a shelly internal coat, nucleus subcentral.

1. B. tentaculata. Cyclostoma impura, Sturm, t. 124. f. 8; Chiaje, t. . f. .

2. B. viridis. Cyclostoma v., Sturm, t. 124. f. 7.

4. Nematura, n. 207. Operculum shate, thick, shclly; side . a nucleus subcentral; shell month contracted. I. N. Deltr. 2. N. polita.

в. Shell spiral, free. Gill inclosed, in one or three lines on left side of mantle-cavity. Operculum spiral. Teeth few, central 1 , lateral $3 \cdot 3$, converging.

Fam. 12. Littorinida.

Foot moderate, for walking; trunk produced, wrinkled, not retractile; tentacula far apart on side of hearl; gills 2, one very large, occupying nearly the whole surface of the cavity and formed of tlat free plates. Amphibious.

a. Operculum ovate, few whorls.

1. Assiminea, n. 193. Eyes on pedicels, united to the short tentacles.

1. A. Grayi, Berkeley, t. 123. f. 6; Gray, t. 5l.f. 8.

2. Litтorina, n. 194. Eyes sessile.

1. L. littorea. Turbo l., Cuvier, t. 51. f. 4.

2. L. varia. Lit. variée, Eydoux, t. 127 a. f. 2. 
3. I. cuariensis, D'Orb. t. 125. f. 111.

4. I. - I. seriale, Eyylonx, t. 127 a. f. 3.

5. I. Gandichandi, Eydon $x$, t. $127 a$. f. 1.

6. L. peruviana, D'Orb. t. 12:3. f. 4.

7. L. Arancana, $D^{\circ}$ Orb. t. l.2. f. 1.

8. L. Hava, D'Orb. t. 12:3, f. 2.

9. L. diemensis. Turbo d., Quoy, t. 51. f. 3.

10. L. Inteola. 'Tubbo 1., Quoy, t. 5l. f. 2..

11. L. seabra. Turbo angulifera, Quoy, t. 51. f. 1 .

12. L. promidalis. Tubo p., Quoy, t. 51. f. f.

13. L. miliaris, Quoy, t. 123. f. 5.

14. L. punctata. Nlamat, Adnuson, t. 5l. f. 5, with a produced siphon? like a Buccinum !

3. Lithoglyphes, n. 198.

1. I. piscium. Paludina p., D'Orb. t. l21. f. 5.

2. L. Lapidum Paludina L., D'Orb, t, 124. f. 4.

4. HYDROBIA, n. 199.

1. II. parvula. Paludina p., Guilding, t. 123. f. 7.

2. II. ? subumbilicata. Rissoa s., Lowe, t. 123. f. 3.

5. Fossar, n. 206.

1. F. ambigna. Fossar, Adanson, t. 51. f. 7. Fossarus Adlansonii, Philippi, t. 125. f. 7 .

6. Lacend, n. 209. 1. L. canalis, Wiegmann, Arch. 1836, t. \&. f. 5. 2.? L. —, Sars.

7. Truceta, n. 218. Operculum homy, of two whorls. T. montana, Benson.

8. Risella, m. 195= Pagoda, sp. Gray, Fig. Moll. Shell trochiform ; aperture rhombie.

1. R. melanostoma. Trochus manus, Quoy, t.51. f. 11.

2. R. amata. 'Trochus a., Quoy, t. 5l.f. 10; f. 14, variety of preceling?

3. R. lutea. Trochus 1., Quoy, t. 51.f. 12.

9. PAgodus, n. 196=Tecturit, Guerin, Icon. t. 11. f. 1. Oper. ovate, subcircular. P. teetum persicum. P. eoronaria. P. patpillosis.

1. P. verus. Trochus Pagolus, Quoy, t. 82. f. 1,4 .

2. P. - , n. s., Adams, t. 126 ia. f. 4.

b. Oper. circular, many-whorled.

10. Nisa. Shell, inner lip arehed, edentulons; axis perforated.

N. Cunningii. Trochus and Littorina C., Philippi.

11. Moneless, n. 197. Eyes halfway up the tentacles. Pillar lip deeply notcherl.

1. M. retusıs. Trochus tectum, Genelin. Monoronta retusa, Lamk. E. 11 .

2. M. trochiformis. Module trochiforme, Eydoux, t. 126a. f. 1.

3. M. lenticularis.

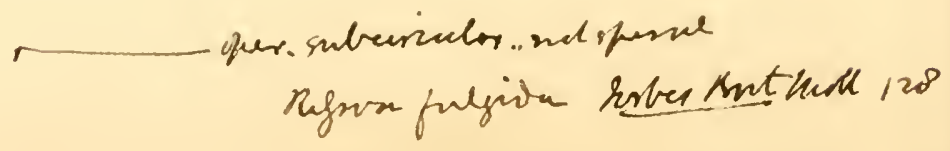


3. xeptexe $=$ anentosa

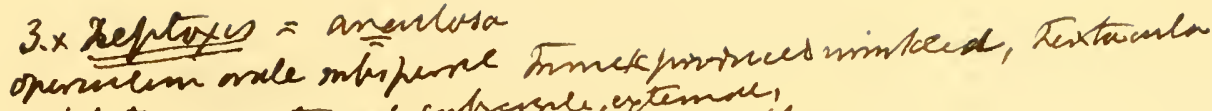
mblate, swes extemse, snherenle, ertemse,

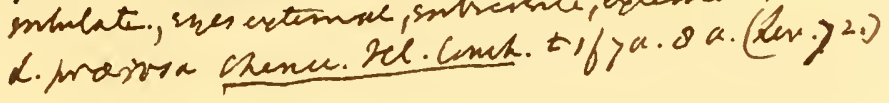




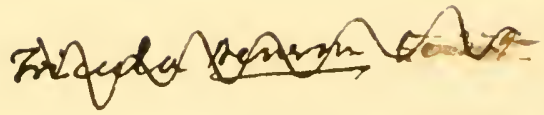

Fonichia Demeren aun othay wH 1851 . 328. 7

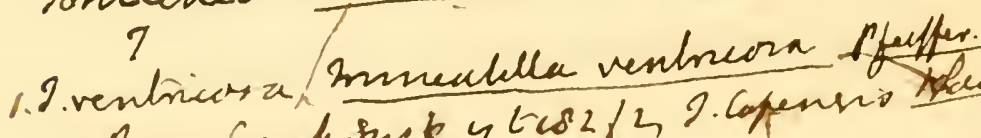

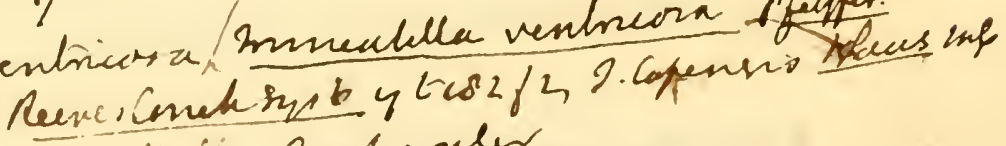
S. Ulin. moti. 2rocthmer 
Fam. 13. Architectomida.

Foot morlerate, for walking. Tentacula near together, ncarly united unizecese Lon at their base, over the retractile trunk. Gill-cavity divided by a longitudinal fold.

1. Architectoma, n. 200 . Oper. ovate, flat, few-whorled; tentacle grooved beneath.

1. A. perspectiva. Solarium p., Quoy, t. 4l. f. 5 ; Eydoux, t. 126. f. 2 .

2. Torinia, n. 200. Operculum circular, elevated, conical, of many whorls. arlange as the moth the shele

1. T. cylindrica. Solarium variegata, Quoy, t. 41. f. 3.

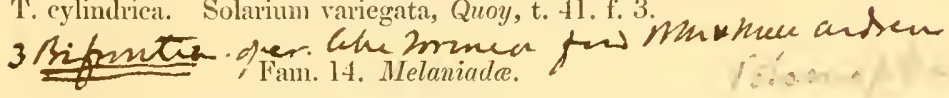

Gill single, composel of rigid cylinctrical plates; mantle torn ou the elge. Tentacula lateral. Trunk anuulaterl, producel, not retractile.

a. Shell white. Oper. ovate, subspiral. Rissoina.

1. RissoA, n. 209. Foot with single dorsal posterior process.

1. R. auriscalpium, Philippi, t. 125. f. 13.

2. R. parva? 'Turbo rissoanus, Chiaje, t. 123. f. 8.

3. R. monodon, Phitippi, t. 125. f. 9.

4. R. ? ineonspicua, Alder, t. 125. f. 5.

2. SKENEA, n. 213.

1. S.?? atomus. Truncatclla a., Phitippi, t. 125. f. 15.

* Operculum ovate, subspiral.

3. Melania, n. 215. Often viviparous.

1. M. armarula, Eydoux, t. $127 a$. f. 6 .

2. M. setosa, Quoy, t. 55. f. 2, 11 .

3. M. virgulata, Eydoux, t. 127 a. f. 8.

4. M. erytlurostoma, Quoy, t. 55. f. 3.

5. M. celebensis, Quoy, t. 55. f. 4.

6. M. trimeatuła, Quoy, t. 55. f. 5, 12.

7. M. uniformis, Quoy, t. 55. f. 6.

8. M. spinulosa. Quoy, t. 55. f. 7.

9. M. costata, Quoy, t. 55. f. 8.

10. M. granifera, Quoy, t. 55. f. 9.

11. M. molluccensis, Quoy, t. 55. f. 10.

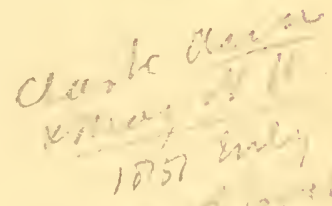

4. Vibex, n. 221.

1. V. aurita. Melania aurita, Rang, t. 54. f. \&.

2. V. Owenii. Melania tuberculosa, Rang, t. 54. f. 1.

5. FAUNUS, n. 229.

1. F, ater. Pirena terebralis, Quoy, t. 55. f. 1. 
** Oper. subcircular or circular, many-uhorled.

6. Melavatru, n. 222. Oper, subsemicirenlar, of few rapidly enlarging whorls.

1. II. fluminea.

2. M. indica. Melanie indienne, Eydoux, t. 127 a. f. 7 ; Voy. Bon. t. 31. f. 12, 15.

3. II. - II. de Tourame, Eydoux, Ioy. Bon. t. 31. f. 47.

4. II. ? Adams, t. 126 a. f. 7. "Oper. circular, of many whorls."

? 7. Clionella, u. 230. Operenlum -?

1. C. simuata. Pleurotoma huccinoidea, Quoy, t. 9(). f. 5.

c. Cerithina.

* Operculum ovate.

8. Runochavis=Cerithium, pars, n. 233.

1. R. diemensis. Cerithium d., Quoy, t. 54. f. 5.

2. R. vertagus. Cerithium v., var. Quoy, t. 54. f. 3.

3. R. aluco. Cerithium a., Quoy, t. 54.f. 7.

4. R. vulgatus. Cerithium v., Philippi, t. 53. f. 6 ; Joannis, t. 173.

f. 3; t. 53. f. 8, 9 क ठ ; Chiaje, t. 52. f. 5.

5. R. lemniscatus. Cerithium 1., Quoy, t. 52. f. 9.

6. R. brevis. Cerithinm h., Quoy, t. 52. f. 8.

7. R. morns. Cerithium m., Quoy, t. 52. f. 7 .

8. R. Tourannii. C. de Tourame, Eydoux, t. 127 a. f. 9.
9. Ceripinasis, 11. 227. C. sulcatis, Say.

** Oper. semicircular, of few rapidly enlarging whorls.

10. Ceritinum, n. 2:33, pars.

1. C. nodulosum, Quoy, t. 52. f. 9, t. 54. f. 6.

2. C. lave, Quoy, t. 52. f. 6.

*** Operculum circular, of many whorls.

11. Bitrium, n. 2:34.

1. B.? reticulatum, Chiaje, t. 54. f. 5, t. 127 . f. 4 .

2. B.? diemenense, Quoy, V.A.t. 55. f. 13.

12. LAMPANia, n. 223. L. zonalis. Cerithium z., Lam.

13. Ceritumea, n. 236 . Oper. eircular.

1. C. oltusa. Cerithiun o., Quoy, t. 52. f. 2, t. 267. f. 3; Eydoux, t. 127 a. f. 10 .

2. C. Kieneri, Quoy, t. 267. f. 4.

14. Trmpanotomes, n. 237.

1. T. fuseatus. Popel, Adanson, t. 53. f. 3.

15. Telescopium, n. 238.

1. T. fuscum. Cerithium 'Teleseopium, Quoy, t. 54. f. 2, t. 267. f. 1 ; Jowe, t. 127. f. 5 . 


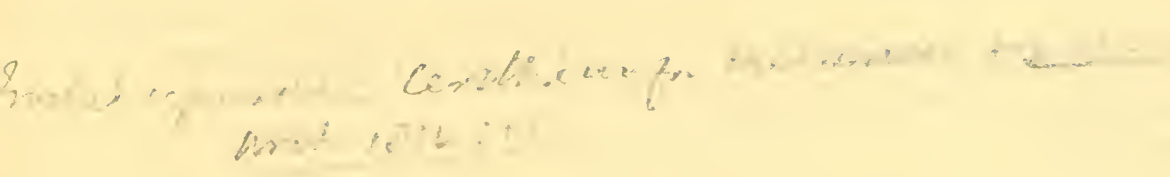




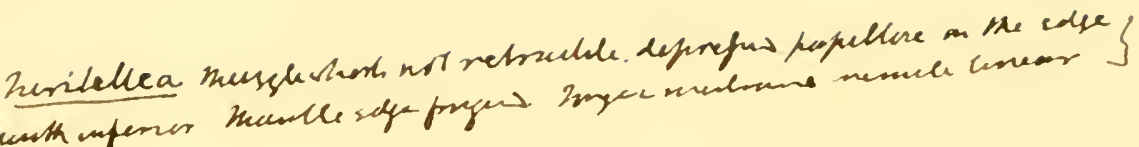
resth anfers 
16. Pyrazus, n. 240.

1. P. palustris. Cerithium p., Quoy, t. 54. f. 4, t.267.f. 2; Kiener, t. 127. f. 6.

2. P. ebeninus. Cerithium e., Quoy, t. 52. f. 4.

3. P. sulcatus, Quoy, t. 52. f. l.

d. Nape crested. Triphorina.

17. Triphoris, n. 241. Oper. circular.

l. T. perversus. Mulex Sarignyus, Chiaje, t. 53. f. 2.

e. Zariana.

18. Terebellum, n. 242. Oper, circular; elge of whorls striated, fringed.

1. T. commune (Turritella terebra), Chiaje, t. 53. f. 7.

2. T. triplicatum. Turritella t., Philippi, t. 128. f. 6.

3. T. ? roseum. Turritella r., Quoy, t. 53. f. 1 .

19. Zaria, n. $244=$ Epitonium, sp. Mus. Berl. 1818 .

1. Z. duplicata. Turritella d., Kieuer, t. 128. f. 7 .

Fam. 15. Naticida.

Foot very large; chin produced in front; shell sunk into the foot. Muzzle elongated, entirely retractile. Opcreular mantle very large.

a. Oper. horny, with a calcareous inner layer.

1. Natica, n. 180.

* Oper. flat, with many spiral grooves.

1. N. Canrena, Cuvier, t. 50. f. 2.

2. N. hebræa, Martyn.

** Oper. flat, with two or three marginal grooves.

3. N. Marochiensis, Quoy, t. 49. f. 3 ; var. t. 49. f. 4, t. 50 . f. 4.

4. N. maculosa, Eydoux, t. 122. f. 2.

5. N. Clausa, Sow.

*** Oper. with a large convex ridge.

6. N. fulminata, Lamk.

2. Naticopsis, $M^{\circ}$ Coy. Operculum calcareous.

b. Oper. horny, simple.

3. Neverita, n. 182.

1. N. glaucina. Natica glancina, Philippi, t. 12. f. 12; Joannis,

t. 119. f. 5; Chiaje, t. 50. f. $1,3,6$.

2. N. - - Natice glauque, Eydoux, t. 120.

3. N. - - N. Chemnitzii, Eydoux, t. 122. f. 6.

4. Lunatia, n. 183.

1. L. limbata. Natica 1., D'Orb. t. 118. f. 2.

2. L. plumbea. Natica 1., Quoy, t. 49. f. 2. 
5. POLINICES, 11 . 185.

1. P. manmilla. Naticam., Ehrenb. t. 50.f. 5 ; Eydoux, t. 122. f. 3.

2. 1'. - ? N. Jaune roux, Eydoux, t. 1.2. f. I.

3. P.? Cher. N. Uher, D'Orb. t.

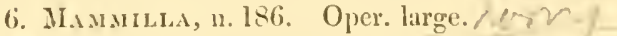

1. M. melanastoma. Natica m., Quoy, t. 4\%. f. 1.

․ II. melanastomoidea. Natiea m., Quou, t. 4.9. f. 5.

3. 1I. fibrosa. Natice fibreuse, Eydoux, t. 12:2. f. 4.

7. Stonatia, n. 19l. Oper. very simall.

1. S. Haliotoides. Cryptostoma Leachii, Gray, t. 11?) f. 3.

2. S. zonalis. Cryptostoma z., Quoy, t. 49. f. 6 ; ? Adams, t. 82. f. 2?.

3. S. Crmba. Cryptostoma eoncarus, Gray, t. 119. f. 3; D'Orh. t. I18. f. 1 .

4. S. lavigata, Eydoux, t. 122. f. 5.

5. S. Reeresii. China, Reeres, t. 12l.

\section{? lam. 16. I'elutinida.}

Mantle edges inflated, folled on the edge into two eamals. Herter solvois - retractile, strong. Tentacules united by a reil. T'ongue linear. 'Teetl, eentral 1 , lateral $3 \cdot 3$, uncinate. Opurculum none.

1. Velutisa, n. 255. Gray, Med. Rep. 1821.

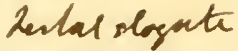

l. V. lavigata. Bulla velutina, Müver, t. 109. f. 10. Velutine Capuloisle, Blainv. t. 10:3. f. 6.

c. Shell attuched, irregular. Gills enclosed in a line on left side of mantle-cavity. Foof scarcely fit for ualking, end dilated, clarute. Operculum horny, circular, or wanting. Teeth

\section{Fam. 17. Vermetida, 1) 156.}

Muzzle produced, contractile, not retractile. Tentacula lateral.

1. Simovicu=Vermetus, part, n. 257. Oper. large, smooth, circular, concave; sear ceutral, aireular, rugose.

1. V. maximum, Gray, t. 128. f. 2. V. giganteus, Quoy, t. 56. f. 5.

2. V. Tollg:umun, Quoy, t. 56. f. 7 .

3. V. reticulatum, Quoy, t. 5 ti. f. 2 .

4. V. cariuatum, Quoy, t. 56. f. 1.

5. V.??--, n. s., Adams, MSS. t. 82. f. 1.

2. Vermetus, n. 257. Opereulum large, rather eonrave; whorls many, thin, with a thin produced extemal edge; sear central, eircular, with close, regular, coneentric grooves. $\mathrm{V}$. Lumbricalis, Lamk. V. Ilindsii. lrregularly twisted, B.M.

3. Broxis, n. 258. Opereuhm rulimentary, small (spiral?).

1. 13. glomerata. Vermetus g., Philippi, t. 58. f. 4.

2. B. triquetra. Vermctus t., Philippi, t. 58. f.5.

3. B. semisurecta. Vermetus s., Philippi, t. 58. f. l.

4. 13, subeancellata. Vermetus s., Philippi, t. 58. f. 3. 
restaule ves shot sees

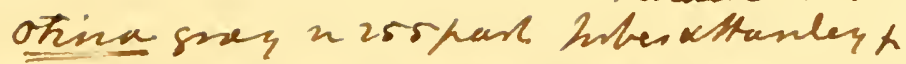
subrotese

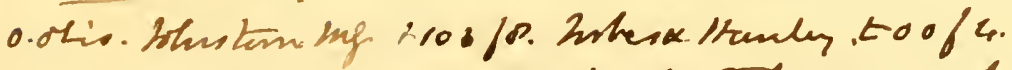

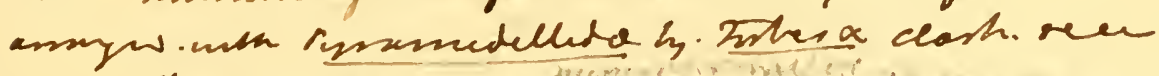

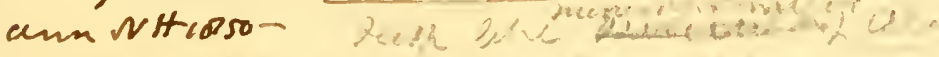

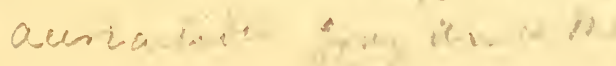



4. Serpuloides, n. 259. Operenlum none; foot truncate, radiated at the tip, front of foot produced into tentacula.

1. S. arenaria. Vermetus gigas, Philippi, t.57. f. 2; Chiaje, t. 58. f. 6 .

2. S. inoperculata. Vermetus i., Riippell, t. 57. f.1.

3. S. dentifera. Vermetus d., Quoy, t. 56. f. 8.

4. S. varians. Vermetus v., D'Orb. t. 128, f. 1 .

5. Lementina, n. 260. Opereulum none; foot truncate, cireular at top.

1. L. Cuvieri, Risso, t. 57. f. 3, probably only a bally deseribed and figured Serpuloides arenarius.

6. Cladopoda. Opereulum none; foot elongate, front end simple, hinder extremity oblong, clavate or subtruncate.

1. C.grandis. Vermetus arenarius, Quoy, t.56.f.9. V.grandis, Gray.

2. C. elegans. Vermetus e., Quoy, t. 56. f. 4.

3. C. nova zealandica. Vermetus n., Quoy, t. 56. f. 6.

7. Siliquaria, n. 261. Oper. spiral, fringed; foot, end truneate, eireular.

1. S, anguina, Philippi, t. 58. f. 2.

2. S. rosea. Vermetus r., Quoy, t. 56. f. 3.

D. Shell free, subspiral, young regularly spiral. Gills form an oblique line across the mantle-cavity, lamine elongate, linear. Operculum none. Muzzle elongate, not retractile. Tonguemembrane winged on each side in front. Teeth, central 1, lateral $3 \cdot 3$.

Fam. 18. Capulide, p. 157.

Foot folded on itself. Egg-eases membranous, attached in a tuft at the front of the foot.

1. Capulus, n. $263=$ Sphondylus, Sloane, 1725.

1. C. ungaricus. Patella hungarica, Curtis, MSS. t. 268. f. 12.

2. IIrpponix, n. 264.

1. H. radiatus, Quoy, t. 130. f. 6 .

2. II. foliatus, Quoy, t. 130. f. 5 .

3. Amalthea, n. 265.

1. A. eoniea. IIipponyx australis, Quoy, t. 130. f. 7.

Fam. 19. Calyptraida, p. 157.

Foot expanded.

1. Crypta, n. 268.

1. C. costata. Crepidnla e., Quoy, t. 58*. f. 2.

2. C. maculata. Crepidula m., Quoy, t. 58*. f. 4.

3. C. aculeata. Crepidula a., D'Orb. t. $58^{*}$. f. 5.

4. C. patagonica. Crepidula p., D'Orb. t. 58*. f. 6.

5. C. Adolphæi. Calyptraa A., Lesson, t.130. f. 4. Animal in shell the wrong way. 
2. GALERUS, 21. 26\%.

1. G. chinensis. Calyptran sinensis, Deshnyes, t. 1301. f. 1; Forbes s. Hanley, B. Holl. t. H13. f. 8-13.

3. Crucibeley, 1. 270.

1. C. lignarium. Calyptræa l., D'Orb. t. 1:30. f. :2.

4. Calyptra, n, 27] = Litheilaphus, Ouen.

1. C. equestris, Owen, Trans. Zool. Soc.

5. Тносніта, n. 272.

1. T. radians. Crepidula r., D'Orb. t. 58*. 1. 3.

2. T. pileus. Crepidula tomentosa, Quoy, t. 5s*. f. 1.

3. T. occidentalis, n. s. Calyptrea chinensis, Guilding, t. 130. f. 2.

? Fam. 20. Phorila, 1. 15ฬ.

Muzzle conical, produced. Foot small, dilated in front. Operculum large, horny, subanunlar.

1. PHorus, n. 273.

1. P. conchylioplioms, Gray, t.

2. Oxustus, $11.274=$ Astralim, Mus. Berl. 1818.

1. O. iudiens, Adams, t. 122 a. f. 2.

2. O. jaranicus, Adums, t. 12: a. f. 1 .

\section{? Fan. 21. J'anicoroidle, p. 156.}

Foot small, circular, produced in front, with a dilated membranaceous expansion on each side. Muzzle conical, wrinliled. Operenlum horny, thin, ovate; whorls few, וр) וper very small.

1. Vanicoko, n. 256.

1. V. Souleytama. Narica S., Reclus, t.123 a.f. 1.

2. V. cancellata. Velutiua c., Quoy, t. 103. f. \&.

E. Shell free, spiral. Gill plumose, exposed; lamine pimnate, spirally twisted. Operculum hormy, spiral; whorls many, with a thin raised edge. Teeth, central 1, lateral :3:3.

lam. 22. Vulvatide, p. 156.

1. VALYTA, n. 25.4.

1. V. cristata, Gruithuisen, t. 126 a. f. 6; Sturm, t. 126. f. 3; Chiaje, t. 126. f. 1?

2. V. plamorbis, Sturm, t. 126. f. 2.

F. Shell free, spiral. Gills inclosed, on left side of mantle-cavity. Operculum spiral, rarely wanting. 'T'eeth numerous, similur (like Bullida and Inthinida). Muzzle elongate, retractile.

Fam. 23. Scalariadee $=$ Scalarina, p. 155.

Teth, ecntral none, lateral many, uniform, hook-like, the onter 


anther larger. Operculum spiral, horny, of few whorls. Tentacula lateral.

1. SCALARIA, n. 248.

1. S. clathris. Turbo e., Leach, t.127. f. 1; Planchus, t.127. f. 7,9;

Kiener, t. 127. f. 2.

2. S. pseudoscalaris, Philippi, t. 127. f. 8.

\section{Seet. 3. Iniophthalma.}

Eyes sessite, on the head between or rather behind the base of the tentacula. Operculum spiral or annular. Passing to Bullidx.

a. Operculum spiral. Eyes near tentacula.

\section{Fam. 24. Cacide.}

Shell subeylindrical, arched, apex deeiduous, subspiral? Oper- nussh culım eircular, horny, spiral. Woot short. Tentaeula simple.Clarke.

1. Cecun, n. 774 .

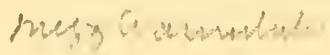

1. C. imperforatum. Junior C. traekea. Oper. concave cxternally. 20 ,

2. C. glabrum. Oper. eonvex externally, with spiral terraces.

\section{Fam. 25. Truncatellida.}

Muzzle broadly 2-lobed. Foot very short, roundish. Opereulum horny. Walks with its foot and lips.

1. Truncatella, n. 282.

1. T. truncatula, Lowe, t. 123. f. 14; Philippi, t. 125. f. 14.

2. T. littorina, Philippi, t. 125. f. 6.

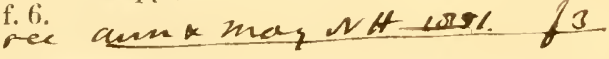

Fam. 26. Pyranidellide.

Teeth none or rudimentary. Opercultum spiral, few-whorled with a thin inner elge.

1. OвеLIscus, n. 283=Plotia, sp. Mus. Berl. 1818.

1. O. maenlatus. Pyramiclella m., Quoy, t. 123. f. 13.

2. O. ventrieosus. Pyramidella v., Quoy, t. 123. f.10; Kiener, t.123.

f. 12. Animal of Quroy's figure of $O$. maculatus placed in this shell!

2. Oровтомiа, n. 286 .

1. O. spiralis, Alder, t. 125. f. 4 ("not correet," Alder). Turbonella s., Lovèn, t. 128 a. f. 3.

2. O. interrupta. Turbonilla i., Lovèn, t. 128 a. f. 4.

3. O. clavula. Tubonilla c., Lovèn, t. 128 a. f. 2.

3. Turbonilla, n. 288.

1. 'T. albula., Menetho a.. Moller MSS. t. 125. f. 2.

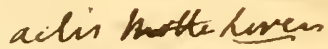

4. Eulina, n. 290 .

1. E. distorta, Philippi, t. 125. f. 11

2. E. polita, Phitippi, t. 125. f. 12. 


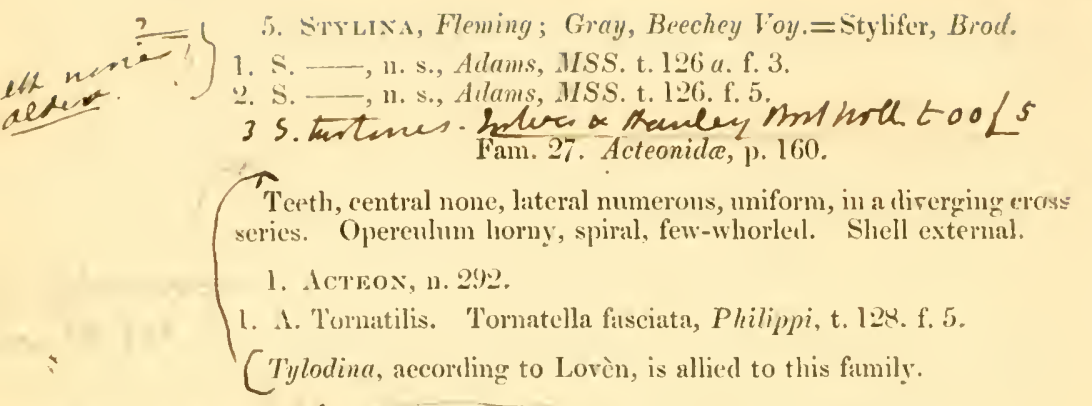
cereur ealenelcentrol b. Operculum anmular. Eyes far behind tentacula.

Fam. ㄱ. ㅇ. Rissoellide.

Front of head nicked, lobes elongate cylinilical, nearly as long as 2. 1.2 alver the tentacula. Eyes far back. Operculum half-ovate, anmular; nu-

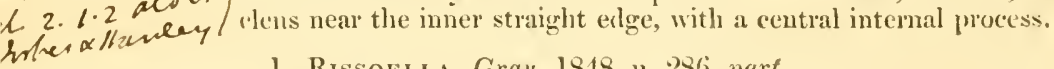

1. Rissoella, Gray, 1848, 11. 286, part.

1. R. glaber. Kissoa? g., Alder, t. 125. f. 3.

? 2 Regina doth persinción.

IR. D'oforder II. Scutibraxchita.

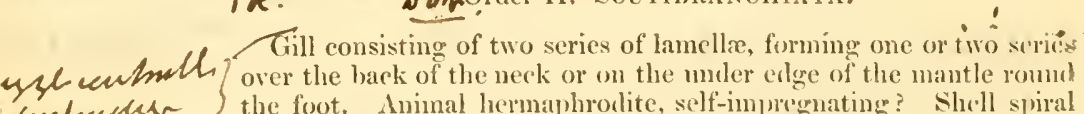
the foot. Animal hermalphodite, self-impregnating? Shell spiral or symmetrical, eonieal. Rectum often traversing the heart.

This order may be thus diviled :-

Normal. IIeart traversed by the rectum; lateral teetlo very ninmerous. Scutibranches, Cuvier, Trochina, Loven. Containing the first seren families.

Abnormal. Ileart separate from the restum. 'Teetls moderate in number, in pairs. Containing the remaining fomilies.

Riuphideg Corsa Invchet Seet. 1. Podophthalma, p. 143.

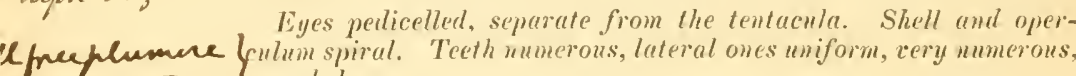
S.rowded.

A. Sides of the foot with a contimued fringe, with three or four cionyated tentacula on its uper surface. Shell internally pearly.

ate centralimallorio

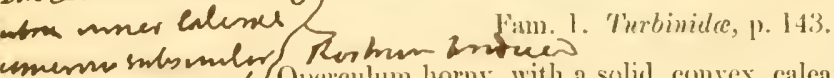

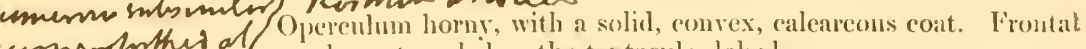

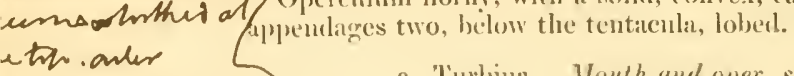

lome ver a. 'Turbina. Moulh and oper, subcircular.

* Oper. without spiral ridges.

1. SARмitress. Oper. extemally coverest with numcrous sepa- 

rate, Hat-topped tubercles. 1. S. Classicarius. Turbo Sar.. Linn. Oper. thin; tubercles separate. 2. S. nataleusis.

Turbo n., Kraus. Oper. thick ; tubercles crowded.

2. Turbo, n. 110 . Oper. externally convex and granular.

1. T. arancanus, D'Orb. t. 108. f. 3 .

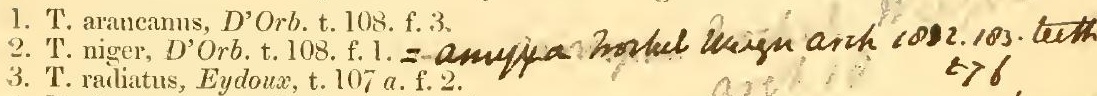

4. T. chrysostomus, Cuvier, t. 41. f. 6.

5. T. marmoratus, Quoy, t. 37. f. I.

6. T. tuberculosus, Quoy, t. 38. f. 5.

7. T. smaragdus, Quoy, t. 38. f. 1 .

$$
\text { ** Oper, with one or more spiral ribs. }
$$

3. Marmorostoma, n. 113. Oper. with an indistinct subcentral rib, surrounded by a broad flat margin.

1. M. undulatum. Turbo u., Quoy, t. 38. f. 2, 3, t. 60. f. 9-14.

2. M. versicolor. Turbo v., Quoy, t. 38. f. 4, 6.

4. Modemid. Oper. with a convex, subcentral granular rib, and a sharp-edged submarginal keel. 1. M. granosus. Turbo g., Martyn.

5. Callopoma. Oper, with a broad central and three or five submarginal spiral ribs, outer ribs toothed. 1. C. fluctuosum. Turbo fl., Gray. Oper. ribs 5. 2. C. saxosum. 'Turbo s., Gray. Oper. ribs 3.

6. Ninella. Oper. roncave externally and edged with two raised spiral ribs and a thin edge. 1. N. lamellosa. Turbo 1., Brod.

2. N. torquata. Turbo t., Quoy, t. 39. f. 2.

7. Collonia. Tuba, n.114, not Lea. Oper. circular, many gradually enlarged whorls, with a convex external rib and central pit. 1. C. marginata. Delphinula m., Lamk. 2. C. striata, Gray. Shell red, white marbled, striated. Africa.

b. Imperatorina. Mouth subquadrate, oper. oblong or ovate.

8. Uranilla, Gray. Oper. Hat, with two distinct convex radiating ribs, lower one straight and nearly parallel to the elge, upper arehed, subcentral, axis imperforate, callous. 1. U. oliracens. Trochus o., Gray. 2.' U. mguis. Trochus n., Gray.

3. U. fimbriatus, Trochus f., Quoy, t. 38. f. 9.

9. Calcar. Oper. thick, with a slight convexity over the axis, outer erlge thin, axis perforated. 1. C. magnus, Chemn. Trochus Calcar, Linn.

2. C. stellaris. Trochus s., Quoy, t. 39. f. 4.

10. Pomaulax. Oper. flat, with three convex racliating ribs; upper submarginal, axis perforated. 1. P. undosus. Trochus u., Gray. 2. P. japonicus. Trochus j., Dunker.

11. Gulldfordia. Oper. flat, with a very slight rislge near the = Astinlim neel evay hal 
arehed or outer margin. 1. G. trimmphans. Trochus Guildfordie, Gray.

2. G. heliophorus. T. imperialis, Quoy, t. 40. f. 1.

12. Pacurroma. Oper. oblong, subquadrangular, very convex externally. 1. P. inxqualis. 'Trochus i., Martyn. Trochus giblberulus, Chemn. 2. P. eaclatus. Troelus c., Gmelin.

13. Lithopoma. Oper. ovate, thick, thicker at the end, with a broad thin margin on outer erge. 1. L. tuber. Trochus t., linn.

14. IMUERATOR, n. 115. Oper, thin, flat, rather thinner on elge. 1. 1. solaris.

15. Tubicantucs, Swains. Oper. ovate, with a slight tuberele over axis, and a single subeentral arched rib. 1. T. Cookianus. Trochus C., Quoy, t. 40. f. 2.

1(j. Bolma. Oper. ovate, suborbienlar, convex, with a subeentral tuberele over axis and a broad submarginal spiral rib.

1. B.rugosus. Trochus r., Limn.; Joannis, t. 108. f. 5; Chiaje, t.107. f. 1,5, t. 43 . f. 6 .

\section{c. Eutropiana. Mouth and oper, ovate.}

17. Eutropia, n. 116.

the notrand - 1. E. anstralis. Phasianella bulimoides, Quoy, t. 41. f. 1, 2. (Cucier, Aluat. Moll. t. .) epulles

Rosm harduce

Fam. 2. Liotiade.

Opereulum hony, with an external ealeareous coat formed of numerous separate pearl-like shelly particles placed in spiral lines.

1. Liотіa, n. 136. Shell varicel; lijs thick. 1. L. gramulosa. Delphimula g., Dunker. 2. L. Kransii. Solarimn eaurellatum, Kraus. 3. L. cancellata. Delph, c., Gray=D. Cobiensis, Reeve.

2. Lippistes, n. 248, part. Montf:=Cyclostrema, Leach. Shell subdiscoidal; month round, lipss scirecly reflexed. 1. L. cancellata. Cyclostrema c., Leach. 2. L.evoluta. Delph. c., Reeve.

3. An Eorus, n. 140. 1. A. subcarinatus. Trochus s., Montague.

Rormen Anatuis

l'ann. 3. Trochide.

Opereuhum homy, of many gradually enlonging whorls, not corered with a calcaleons coat. No fromtal fringe between the tentacula.

$$
\text { * Operculum circular, many-whorled. }
$$

1. PYRAM1s, 11.117.

1. P. obeliseus, 'Trowhus o., Quoy, t. 39) f. 3.

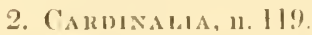

1. C. clata. Trowhus e., Quoy. t. fil f. 22, 26, 





$$
\nu \text { : . }
$$

20.? 
3. Trochus, n. 120.

1. T. niloticus, Quoy, t. 43. f. 1.

4. Polydonta, n. 122.

1. P.? tiarata. Troehus t., Quoy, t. 61. f. 6, 11.

2. P. sandwichensis. Troque sandwiehien, Eydoux, t. 107 a. f. 5.

Frontal appendage distinet, lobed! .

5. Clanculus, n. 123. Forehead with a toothed erest on each side near tentacula? C. oruatus. Tr. Clanculus, Gray.

1. C. Pharanis. Trochus Ph., Cuvier, t. 42 . f. 3.

2. C. patagonieus. Troehus p., D'Orb. t. 48. f. 2.

3. C.? Isabellæ. Trochus I., D'Orb. t. 108. f. 4. Sotur $=4$. C. limbatus, Quoy, t. 63. f. 1, 6 .

6. Ziziphinus, 1. 124.

1. Z. vulgaris. Tr. ziziphinus, Chiaje, t. 43. f. 3, t. 107. f. 2 ?

2. Z. erythroleneos. Tr. e., Chiaje, t. 107. f. 3.

3. Z. connlus? Chiaje, t. 107. f. 2.

4. Z. oranulatus? Chiaje, t. 43. f. 2.

5. Z. diaphanus. Trochus d., Quoy, t. 64. f. 1-5.

7. Canthiridus, n. 125.

1. C. irisidontes. Troehus i., Quoy, t.125. f. 8. - autrath.

8. Monodonta, n. 127.

1. M.? osilin, Desh. Osilin, Adanson, t. 43. f. 7, 8.

2. M. treniata. Trochus t., Quoy, t. 63. f. 15, 17.

3. M. eanalifera. Trochus c., Quoy, t. 64. f. $26,29$.

9. LaBio, n. 128.

1. L. fagaroides. (No name), Chinje, t. 42. f. 7.

2. L. - ? (No name), Chiaje, t. 108. f. 6.

3. L. eonstrictus. Trochus c., Quoy, t. 63. f. $23,27$.

4. L. striolatus. Trochus s., Quoy, t. 63. f. 18, 22.

5. L. zelandicus. Trochus z., Quoy, t. 64. f. $12,15$.

6. L. cingulatus. Trochus c., Quoy, t. 64. f. 16, 20.

10. Chlorostoma, n. 129=Oxystele, Philippi, 1847.

1. Ch. merula. Troehus m., Chiaje, t. 42. f. 8 .

\section{Hetetna, 1t, 130.}

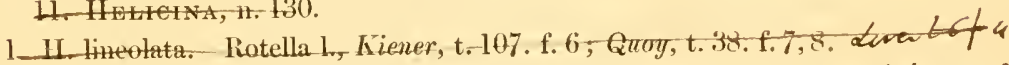

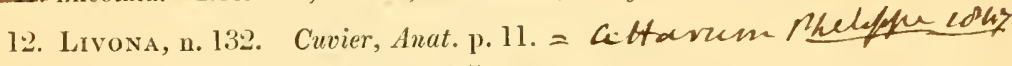

1. L. Pica. Turbo P., Cuvier, t. 41.f. 7.

13. Crclostoma, n. 135.

1. C. Delphinus. Delphinula laciniata, Quoy, t. 43. f. 4, 5 ; Kiener, t. 107 . f. 7 .

2. C. nigrum. Dauphinule noire, Eydoux, t. 107 a. f. 1.

14. GiввuLd, n. 137.

1. G. cincraria. Troelıus c., Müller, t. 42. f. 5,6 ,

2. G. —— Chiaje, t. 42. f.9. 
3. G. - ? Chioje, t. 12. f. 2.

4. G. ?? - C Cuvier, t. 42. f. 4.

5. G. - ? Chiaje, t. 42. f. 1.

6. G. - - ? - t. 5l. f. l, 2.

15. Philippia, n. 112. 1. P. lutea. Solarium 1., Philippie. Moll. Sicil. i. 174.

** Operculum ovate, few-uhorled.

16. Aradasia = ? Otaria, n. 131. Opereulum ovate, of few rapidly enlarging whorls; lateral filments $(4 \cdot 4)$ elongate; shell scareely pearly.-Quoy. 1. A. canaliculata. Trochus e., Quoy, t. 64. f. 21,23.24. = inchelew rhilles cong

Astrmm Fom. 4. Stomatellida, Syn. 13. M. 1842.

Operenlum horny; whorls many, gradually enlarging; sometimes wauting. Frontal lobes between the tentacula two, listinct, lobed. Iantle and sliell entire; siles without filaments. Gill single, lateral fringe membranaeeous, without tentrenla. 'Teeth, Quoy, t. $66^{*}$. f. 19.00:4:4.00

thele sonlute 1. Stomatella, n. 143. -Operenlum horny, cireular, manywhorled; mantle elge crenated.

1. S. maculata, Quoy, t. 109. f. 1, 3.

2. GENA, n. 145. Operenlum none; shell elongate ear-shaped; mantle erlge entire.

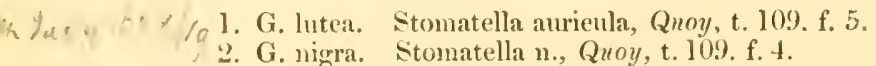

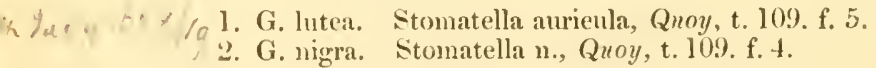

Fam. 5. Haliotida.

Reshromination

Operenlum none; frontal lobes none? Mantle with a slit and shell with a series of holes on pillar sicle. Gills 2, sejarate on pillar side.

1. IIALiotis, n. 147.

1. H. tuhereulata, Cuvier, t. 109. f. 6, t. 110. f. 5 ; Chiaje, t. 109. f. 2, $2 a ; 11$ elb. t. 110. f. 7 .

13. Sides without membranous fringe or tentacula. Shell not ectatore ronoll or nume. nur laleral mequneximer Fam. 6. Neritinide.

ne ven enat. Teeth, eentral few, with mumerous lateral hooks, like the former ter lalerne fumilies,-Loven, t. 6. f. 5 .

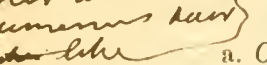

a. Operculun hormy, with a shelly cont on each side; edge channeled.

were the

xalementa

1. Nerita, n. lit.

ThEuyt65/30.1. N. plicata, Quoy, t. 45. f. 6.

2. N. allicella, Quny, t. 45. f. 5 .

3. N. exuvia, Cuvier, t. 14. f. $T$.

4. N. - - Dumar, Adamson, t. 11. f. 5. 6.

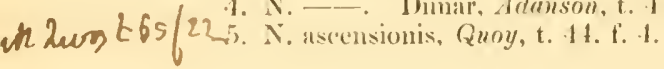



6. N. versicolor, Quoy, t. 44. f. 2, 3.

7. N. pumctata, Quoy, t. 44. f. 1.

8. N. polita, Qnoy, t. 45 . f. \&.

9. N. L?, Lamarck, MSS. t.

beeth heer 6 65/26. (frot aldu)

10. N. lineata, Quoy, t. 265. f. 4.

b. Operculum solid, shelly, with a thin flexible edge.

2. Neritella, n. 161=Lamphrostoma, Rafin. Oper. as large as month of shell.

1. N. pulligera. Nerit. p., Quoy, t. 45. f. 4.

2. N. reticulata. Nerit. r., Quoy, t. 45 . f. 1.

3. N. brevispina. Nerit. b., Quoy, t. 45. f. 2.

funzeler Liven $66 / 5$

4. N. communis. Nerita c., Quoy, t. 45. f. 3.

5. N. strigillata. Nerita s., Quoy, t. 45. f. 7.

6. N. amphibia. Nerita a., Lesson, t. 45. f. 9.

7. N. virginea. Nerita v., D'Orb. t. 128 . f. 3.

8. N. Georgiana. Nerita G., Eydoux, t. 106 a. f. 3.

9. N. Gaimarti. Nerita G., Eydoux, t. 106 a. f. 2.

10. N. Michandi. Nerita M., Eydoux, t. 106*. f. 1 .

11. N. picea. Nerita p., Eydoux, t. 106*. f. 4 .

12. N. vestita. Nerite vêtue, Eydloux, t. $106 \alpha$. f. 5 .

13. N. Goldii. Nerita G., Eydoux, t. 106 a. f. 7.

14. N. indica. Nerita i., Eydoux, t. $106 a$. f. 8.

15. N. rugosa. Nerita r., Eydloux, t. 106 a. f. 6.

16. N. tahitensis. Nerita t., Eydoux, t. 106 a. f. 10.

17. N. Nuttallii. Nerita N., Eydonx, t. 106 a. f. 9.

3. Catillus, n. 165 . Oper. small, rndimentary.

1. C. porcellanus. Navicella elliptica, Blainv. t. 46. f. 5, 6; Qnoy, tsof32 t. 46 . f. 12, 34 .

\section{Sect. 2. Edriophthalina.}

Eyes sessile, or on a slightly raised tubercle on the onter side of the base of the tentacula. Shell (adnlt) symmetrical conical, not spiral; not pearly internally. Operculum none.
ath centrie 1.: thit. cornis
en talusce Foot dilated; npper side with a series of short tentacles. Borly
and shell short, broad, conic. Gills 2, separate, symmetrical. 'h

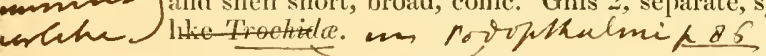
Gills 2, symmetrical on back of neck.
Rhepactrgerst he
a. Shell external or partly naked.

1. Scutus, n. 152=Dascims, Rufinesque, 1815. (Teetl, Quoy,

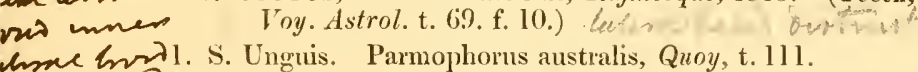

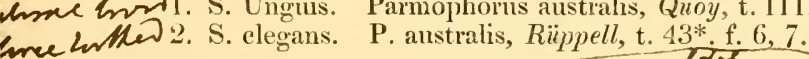

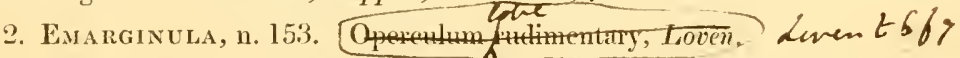

\section{* Mantle inclosed.}

1. E. fissura. Patella f., Mïller, t. 70. f. 9; Chiaje, t. 113. f. 5,

t. 70. f. 4. Var. E. rosea, Forbes, B. M. t. BB. f. 1, 2, 3.

2. E. elongata, Phitippi, t. 110 . f. 3. 
** Manlle much erpanded, parlly coveriny the shetl.

3. F. Cuvieri, Aud. Sacigny, t. 114. f. s; Cuvier, t. 114. f.

3. Puncturella, n. 155.

1. P. Noachina, Forbes \&) Hanley, Brit. Holl. t. Bl3. f. 4, 5, 6.

Th certroe small 4. Fissurel.A, n. 156.

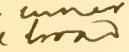

\section{* Mantle inclosed.}

1. F. costata, Lesson, t. 43*. f. 1-4.

2. F. ractiosa, Jesson, t. $43^{*}$. f. 5, t. 113. f. 1 .

3. F. gracra? Chiaje, t. 112. f. 1, t. 113. f. (;, 7. Young, Chiaje, t.11:3. f. 4 .

4. 1'. patagonica, D'Orb. t. 113. f. 2.

5. F. maxima, D'Orb. t. 113 . f. 3.

** Mantle reflexed on edye of shell.

6. F. Cuvieri, Cuvier, t. 110. f. 6.

b. Shell permanently covered by the mantle.

5. FissurfllideA, n. 158.

1. Fissurellidaca liatula. F. megatiema, $D^{\prime} \mathrm{Or} b, \mathrm{t}, 112$. f. ‥

6. Pupillia, n. 159.

1. P. apertura. Pupillaen aperta, Krans, t. 269. f. 5.

7. Lucapina, n. 160. I. L. cancellata. 2. L. crenulata.

Foot small, conical; siles simple. Borly and shell clongate conral ; apex piereed.

1. Dentalium, n. 277 .

1. 1.? semistriolatum, Limn. Trans. t. 115. f. 4; Chiaje, t. 115. f. 3.

2. Entalis, n. 278.

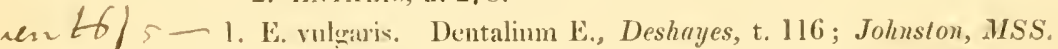

t. 115. f. 2 .

codrumitur hart B. Gill single, on side of the back of the neck. Foot with a simple honchat impressed groove. Teeth few, subequal, in pairs.

\section{Fam. 9. Tecturidce, p. 158.}

\section{rolelleax $x$ heven.}

Teeth, entral 2 , with 2 lateral on each side in an oblique line, inuer one often larger. Gill singrle, on sible of the back of the neck.=l'ittellea b., Loven.

1. 'Тестия, 11. 275.

1. 'T. parva, Patella virginea, Miiller, t. 70 . f. 7, t. $111^{*}$. f. 7 . hechequal "2. 'T'. tessellata. Patella t., Miiller, t. 70. f. (i.

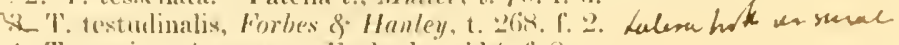

4. T. rassis. Armata c., Eschselh, t. 11 1. f. \&.

5. T. H:(m)

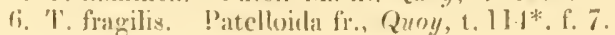


cestonekar

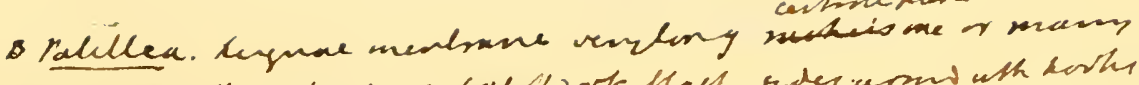

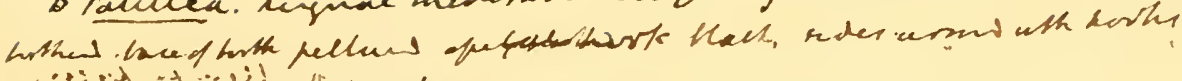

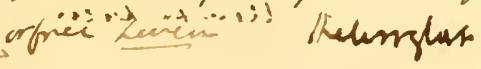

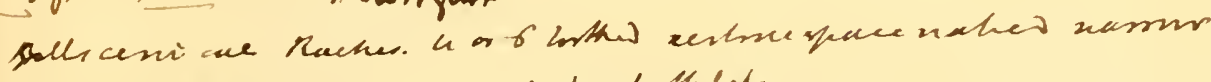
reperesupheren. soler buthlef 


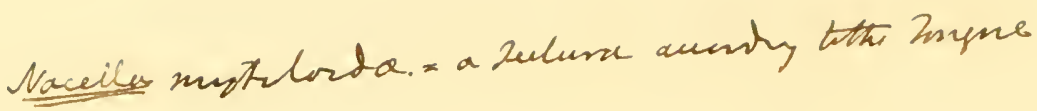

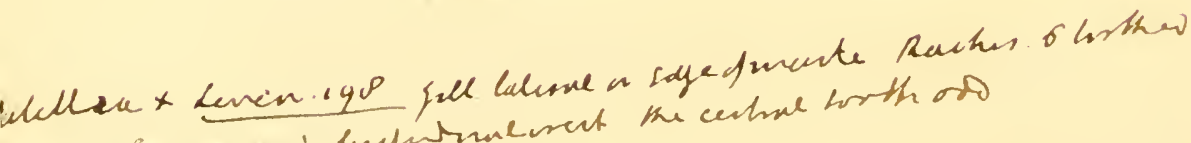

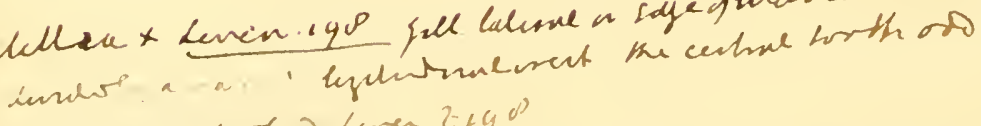
Laluma. 3. 3. hathes deren $2<y 0^{\circ}$ 
7. T. striata. Patelloida s., Quoy, t. 114*. f. 6.

8. T. stellaris. Patelloila s., Quoy, t. $114^{*}$. f. 4.

9. T: coniea.' Patelloida c., Qyoy t. $114^{*}$, f. 2.

10. T. punctata. Lottia 1., D'Orb. t. $114^{*}$. f. 3." *. . .

11. —, Müller, Z. D. t. 110*. f. 9.

2. Scurria, n. 276.

1. S. mitra. Acmæa m., Eschsch. Lottia Scurria, D'Orb. t. 114. f. 1 .

Fam. 10. Lepetide. $=$ ral. Cryplobrenchea

Mindend

Tooth, central single, with two hook-like teeth on each side.

Gills none? or 2 pimnate on back of neck. Patellea c., Lovèn.

1. Lepeta, n. 406=Propilidium and Pilidium, pars, and Iothia,

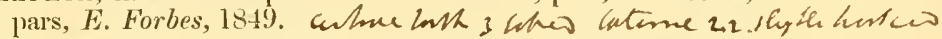

1. L. cæca, Miiller, t. . Propilidium ancyloide, Forbes, t. 268. f.4.

2. Iothis, pars, Forbes, Athencum, 1849. Pilidium, Forbes,

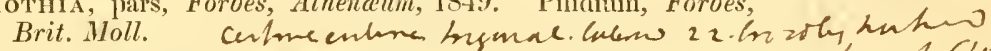

1. Iothia fulva. Patella fulva, Müller, t. 70. f.5. Pilidium fulvum, Forbes, t. 268. f. 3.

\section{Fam. 11. Gadiniade.}

Gill single, obliquely across the back of the neck. Tentacula expanded, forming a funnel-shaped expansion. Eyes sessile. Shell conical, muscular impression horseshoe-shaped, marginal, with a groove (for the vent) in the front of the right side of the scar. Teeth —?

1. Gadrnia, n. 513=Liria, Gray. 1. G. Garnoti. Pileopsis Garnoti, Philippi (Wiegnann, Arch. 1839, t. 3. f. 3).

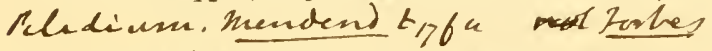

c. Gill on the inner surface of the mantle, forming a more or less complete ring just beneath the margin. Side of foot with a sunken groove.

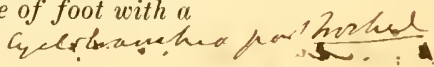

Fam. 12. Patellida, p. 168.

Shell simple, conical. Teeth, central two pair, with 4 lateral, one on each side; inner one larger and lower down. Patellea a., Lovèn.

1. Patella, n. 402.

1. P. compressa, Quoy, t. 70. f. 1.

2. P. granularis, Quoy, t. 70. f. 2.

3. P. scutellaris, Quoy, t. 70. f. 3 .

4. P. cærulea, Desh. t. 70. f. 8 .

5. P. vulgata, Cuvier, t.70. f. 10 ; Chiaje, t. $70^{*}$. f. 1,2 ? 6 ?, t. $110^{*}$. Leven $66 / 8$ f. 3, t. $110^{*}$. f. $1,2,4,5,8$; Fleming, t. $110^{*}$. f. 6 .

6. P. pyramidata, Joannis, t. $70 \%$ f. 3.

7. P. zebrina, $D^{\prime} O r b$. t. $70^{*}$. f. 7 .

8. P. crenata, D'Orb. t. 110. f. 2 .

9. P. Lowei, $D^{\prime} O r b$. t. 110 . f. 1 .

2. Nacelia, n. 403.

1. N. cærulea. P. pellucida, Miiller, t. 70. f. 4; Forbes \& Hanley, Laven 2-6/, t. 268 . f. 1 . 


* Shell covered.

jlaga1sos 1.hDoridum, n. 298.

1. D. marmoratum. Aeera marmorata, Cantr. t. 131.f. 3. D. aplysiæforme, Chiaje, t. l31.f. 2. Eillothea marmorata, Risso (Eur. Merid.t. 1. f. 17).

2. D. Meckelii, Chiaje, t. 132. f. I. D. membranacenm, Meckel. Aceres, Cuv. t. 132. f. 1 a.

2. Philine, n. 299.

1. P. aperta. Lobaria quadriloba, Müller, t. $60^{*}$. f. 2 . Bullæa a., Cuvier, t. 60. f. 5, t. 175 . f. 6 ; Chiaje, t. 132. f. 6 .

2. P. —, n.s. Borneo, Adams, t. 175 . f. 5 .

3. P. —, n. s. West Indies, Guild. t. 132. f. 4.

4. P. granulosa, Sars, t. 60*. f. 4.

3. Hirundella.

1. II. hirundinaria. Bulla h, Quoy, t. 60. f. 1 .

** Shell exposed.

4. Cryptophthalmus, n. 300 .

1. C. smaragdina, Ehrenb. t. 60. f. 3, 4, 5, 6. Var. Rüppell, t. 60*. f. 9 .

5. SCAPHANDER, n. 301 .

1. S. lignarius. Bulla 1., Chiaje, t. 60*.f. 5 .

2. ? 13. —, Cantr. t. 132. f. 3 .

6. Glauconella.

l. G. viridis. Bulla v., Quoy, t. 59. f. 5.

2. G. glanea. Bulla g., Quoy, t. 59. f. 3 .

3. G. Adamsii. Bulla smaragdina, Adams, MSS. t. 178. f. 1.

7. Xanthonella.

1. X. lutea. Bulla l., Quoy, t. 59. f. 8.

8. Alicula, n. 302 .

9. ? Rhizophorus, n. 305 . Teeth, central 1 , lateral $1 \cdot 1$.

10. ? Gasteropteron, n. 313. Teeth - ?

1. G. Mcekelii, Blamv. t. 60*. f. 8; MSS. t. 131. f. l. Clio amate, Chiaje, t. 132. f. 9.1; Cantraine, t. 132. f. 10.

11. ? Atuas, n. 314.

1. A. Peronii, Lesueur, t. 257. f. 3.

\section{Fam. 2. Bullinada.}

Teeth, central none, lateral $6 \cdot 6$, inner ones large, hooked, outer ones uniform, small (rarely wanting). Tentacula broad, lateral, recumbent, transverse. Foot short. Shell exposed.

1. Bullixa, n. 304.

1. B.? - n.s. Borneo, Adams, t. 178. f. 4. 
l'am. 3. Bullide.

'T'eeth, central 1, lateral numerons, nuiform, in an arehed series. Tentacular frontal disk exprunded, nicked behind.

1. AkiRA, n. 303.

1. A. bullata. B. nkera, Müller, t. 60*. f. 7. ? B. fiagilis, Blaim. t. $6(0)$. f. 6 .

2. A. —, n.s. Borneo, Adams, t. 178. f. 2.

2. BULLA, n. 307.

1. B. ampulla, Adams, t. 178. f. 3 .

2. 13. ovoirle:t, Quoy, t. 59. f. 4.

3. 13. glauca, Quoy, t. 59. f. 3.

4. 13. striata, Quoy, t. 60. f. '2; ITiegmam, t. 175. f.4.

5. 13. Columni, Chiaje, t. 59. f. 1 .

6. B. Hydatinclla. B. Iyclatis, Chiuje, t. 60*. f. 3.

3. I1 Mines, Leach. Bulla, n. 307, pars.

1. H. Indatis? D'Orb. t. 60. f. 1, 2. Bulla h., Chiuje, t. 6(t*. f. 2 .

2. II. - Bulla aperta? Guild. t. 132. f. 4.

3. II.? - n. s., Adams, t. 17R. f. 5.

Fam. 4. Amplustrida.

Tecth, central none, lateral uumerous, uniform. Tentacular frontal disk produced into ear-like tentacular lobes. Front of aperture of shell more or less cliameled.

1. Amplustrum, n. 309.

1. A. aplustre, Quoy, t. 59. f. 2. B. banderole, Eydoux, t. 175. f. 1.

2. IYDATINA, n. 310.

1. 11. Physis, Templeton, t. 262. f. 1; Adams, t. 175. f. 3; Ruoy, t. 59. f. 7 ; Eydoux, t. 175. f. 2.

3. BuLliNuLA, n. 311 .

1. 13. uudata, Quoy, t. 59. f. 6 .

2. 13. - Bulliea, Chioje, t. 132. f. 5.

\section{Fam. 5. Aplysiadle.}

Tecth, ecntral 1, lateral numerous, similar. Tentacula separate, ear-like.

\section{Shell covered by the mantle.}

1. Dolabelda, n. 315. Shell uniform, calcareous, triangular; apex callous.

$$
\text { * Warty. }
$$

1. D. callosa, Cuvier, t. 137. f. 2. D. Rumplii, Rany, t. 135; Quoy, t. 131. f. 1.

2. D. - Rang, t. 133 .

3. D. - n. s. Ceylon, Templeton, t. 207. f. 2. 


** S'mooth.

4. D. ecaudata, Rang, t. 136. f. 2 .

5. D. Tongensis (Aplysia de Tonga), Quoy, t. 137. f. I.

*** Shell elongute.

6. D. temnida, Rang, t. 136. f. 1 ; Templeton, t. 270 . f. 1 .

2. Dolabrifera, 11. 316. Shell calcareons, trapeziform; apex produced. hot.lonetertwath, bach slyoly womes

1. D. Dolabrifera, Rang, t. 149. f. 1. Edgay kne Lody.

2. D. ascifera, Rang, t. 149. f. 3.

3. D. - (A. Oalıou), Eydoux, t. 220. f. 5.

3. Aplysia, n, 317. Shell subcartilaginous, ovate; apex acute.

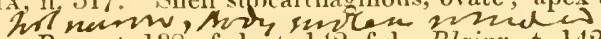

1. A. depilans, Rang, t. 138. f. 1, t. 142. f. 1; Blainv. t. 142. f. 2. Jun.? Esmia Griffitlsix, Leach, t. 268. f. 13. Young from eggs, Quatrefages, t. 154. f. I.

1*. A. virescens, Risso, t. 61. f. 3.

2. A. punctata, Cuvier, t. 139. f. 1; Rang, t. 140. f. 1; Guérin, t.177. f. 5 ; Plilippi, t. 146 . f. 2.

3. A. Lepus, Philippi, t. 146. f. 3.

4. A. marginata, Phitippi, t. 146. f. 1.

5. A. ocellata, $D^{\prime} O r b$. t. 148.

6. A. rosea, Rang, t. 138. f. 2 .

i. A. Lessonii, Rang, t. 139. f. 2.

8. A. brasiliana, Rang, t. 140. f. 2 .

9. A. Keraudrenii, Rang, t. 141. f. 2.

10. A. fusca, Rang, t. 1+1. f. 1 .

11. A. maculata, Rang, t. $142^{*}$. f. 1 .

12. A. fasciata, Rang, t. 142\%. f. 3 .

13. A. Inca, D'Orb. t. 144. f. 1; t. $61^{*}$. f. 2.

14. A. dactylomela, Rang, t. 144. f. 2 .

15. A. protea, Rang, t. l44. f. 1 .

16. A. Camelus, Rang, t. 145. f. 1.

17. A. alba, Rang, t. 145. f. 2.

18. A. Sicula, Swain. t. 147. f. 3.

19. A. nigra, $D^{\prime} O r b$. t. 147 . f. 1.

20. A. Sorex, Rang, t. 149. f. 8.

21. A. Ferussacii, Rang, t. 149. f. 7 .

2.2. A. longicornis, Rang, t. 149. f. 4.

23. A. marmorata, Rang, t. 149. f. 6.

24. A. lurida, D'Orb. t. $61^{*}$. f. 1 .

25. A. Rangiana, D'Orb. t. $61 *$. f. 3.

26. A. Julienna, Quoy, t. 61. f. 1.

27. A. Tigrinella. A. Tigrina, Rang, t. 61**, f. 4.

28. A. petalifera, Rang, t. 149. f. 5 .

29. A. lingnifera, Rang, t. 149. f. 2.

30. A. Argus, Rïppell, t. 143. f. 2; ? Templeton, t. 270. f. 3, 4.

31. A. Brugnatelli, Webb, t. 142*. f. 4 .

32. A. Weblii, Van Beneden, t. 142*. f. 2.

33. A. depressa, Cantraine, t. 147. f. 4.

34. A. Dumortieri, Cantr. t. 147. f. 3.

35. Aclesia limacina, Rang, t. 151. f. 2.

36. Aplysia Lepus. Dolabella Lepus, Risso (Europ. Merid.t. f. ). 37. Aplysia neapolitana, Chiaje, t. 3. f. 2. 


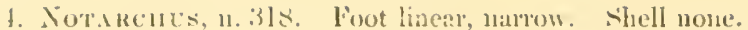

1. X. Curiori, Curier, t. 53. f. 3; Blainville, t. 152. f. 3, t. 15.3. f. 33; Chirije, t. 15:3. f. 1.

2. N. gelatinosus, Rany, t. 153. f. 2. Aplysia g.. Quoy, t. 61. f. 2.

3. N. junctatus, Plilippi, t. 153. f. 5.

4. N. ocellatns, Rang, t. 152. f. 2.

5. ? N. (Bursiris) griscus, Risso, t. 198. f. 8.

5. Bersatella, n. 319. Shell none.

1. B. Leachii, Blainv. t. 153. f. 4. Aclesia Savigniana, Rang, t. 150.

2. 13. laciniatus (Notareluns 1.), Riippell, t. 152. f. 1.

3. 13. I'leii (Aclesia P.), Rumg, t. 15l. f. 1.

6. ACLesia, n. 319*.

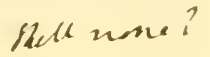

1. 1. longicauda, Rang, t. 151. f. 4.

2. A. Indata, Rany, t. 152. f. 5 .

3. A. citrina, Rang, t. 15l. f. 6.

4. A. virulis, Rang, t. 151. f. 6.

5. A. lufa (Aplysia r.), Quoy, t. 6il. f. 5.

6. A. striata (1plysia s.), Qmoy, t. 61. f. 3.

7. A. cimigera (Aplysia c.), Quoy, t. (il. f. 1 .

8. A. ocellata (l'aciburanchus o.), Adums, t. 179. f. 2.

9. A. Eucloms (1'acobranchus E.), Adams, t. 179. f. 1.

2. Organs of generation close together in one tubercle.

l'am. 6. Lophocercide.

Tentacula 2, car-like. Eves - Gill regular, pectinate. Male organ on right sile of nape, near tentacle (Ann. Sci. Nat. 1847. p. 5.9).

1. LOPHOCERCUS, n. 321 .

I. L. Sicbolılii, Krohn, t. 176. f. 3.

Th 2. LoBiger, n. 322.

1. L. Philippii, Krohn, t. 176. f. 1.

\section{Fam. 7. Pleurobrunchilla.}

1. UMBRELLA, H. 323.

$$
\text { * Shell external. }
$$

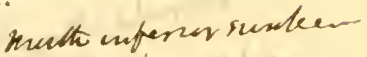

1. U. umbellata. U. ehinensis, Bluinv, t. 163. f. 1, 2. U. imlica, Gray, t. 163. f. 4; Eydonx, t. 165.

2. I. melliteranea, Chiaje, t. 163. f. 3; Cuxier, t. 164.

2. Truomina, n. 324. Perbaps allied to Pyramidellida.

1. T. punctata, Plilippi, t. 103. f. 7. 'T. citrina, Joannis, t. 10;3. f. 1.

** Shell corered by the mantle.

3. PleEROBRAXCHUS, n. $325=$ (BERTHELLA, n. 326.)

* Shell large.

1. P. Peronii, Cüv. t. 167. f. 8; t. 167, f. 1, in spirits; Quoy, t. 167. f. 6, alive. 


2. P. plumula. Berthella porosa, Blainv t. 167 . f. 4.

3. P. perforatus, Philippi, t. 171. f. 4.

4. P. aurantiacus, Risso, t. 167. f. 9, t. 167. f. 5 ; Cantraine, t. 174.

f. 1 ; Philippi, t. 167. f. 3.

5. P. De Iaanii, Cantraine, t. 167. f. 2.

6. P. brevifrons, Philippi, t. 171. f. 3.

7. P. - - t. 169. f. 1 .

8. P. patagonicus, D'Orb. t. 171 . f. 1.

9. P. comutus, Quoy, t. 174. f. 2.

10. P. pumetatus (Quoy, Voy. Ast. t. 22. f. 15, 19).

11. P. tuberculatus, Meckel.

12. P. stellatus, Risso.

13. P. ocellatus, Chiaje.

14. P. - Rïppell, t. 3. f. 2 ; t. 166. f. 1. Lepus marinus, Forskael, t. 169. f. 5.

15. P. Blainvillii, Lesson, t. 172. f. 1 .

16. P. mammillatus, Quoy, t. 174. f. 4.

** Shell very small.

17. P. testudinarins, Philippi (Moll. Sicil.) t. 20, 21. f. 1.

18. P. mammillatus, Philippi, not Quoy.

19. P. Forskalii, Chinje, t. 169. f. 4.

20. P. tuberculatus, Chiaje, t. 172. f. 2.

21. P. reticulatus, Rang, t. 169. f. 3.

**** Shell narrow, shelly?

22. P. oblongus, Savigny, t. 170, 173. f. 1 .

4. Oscanius, v. 325*. Mantle expanded, irregular.

1. O. membranaceus. Lamellaria m., Montague, t. 103. f. 12.

2. O. Lesuenrii. Pleurobranclus L., Blainv. t. 166. f. 2.

3. O. luniceps. Pleurobranchus 1., Cuvier, t. 167. f.2.

5. PleurobranchaA, n. 327.

1. P. Meckeli, Blainv. t. 168. f. 1; Cuvier, t. 168. f. 3; Chiaje, t.173. f. 2 ; Cantraine, t. 173. f. 3, from life. Pleurobranchidium della Chaii, Verany, t. 220. f. 3, young.

2. P. maculata. Pleurobranchidium m., Quоy ('Toy. Astrol.) t.22. f. 12 .

6. Posterobranchaea, n. 318.

1. P. maculata, D'Orb. t. 168. f. 2.

13. Gill on the inner edge of the front of the side of the mantle. Foot small. Animal formed for floating. Allied to the Ptenobranchiata, but the gills are not regularly comb-like, but tufied, as in this Order.

a. Foot compressed, erect, fin-like, with a small flat disk on the hinder edge. Body and shell spiral or subspiral. Teeth, central 1, lateral 3:3, elongate, unequal. Heteroporles, Lamk.

Fam. 8. Pterotracheidce, p. 168.

Body elongate, transparent. Gill, \&c. in a small prominent bag, naked, or covered with a thin subspiral shell. Operculum none. Organs of generation in a common tubercle. 
1. Axops, 1) Orb. n. 331. 'T'entraculn none; eyes none; head inlistinct; slell none.

1. A. Peronii, D'Orb. t. 62. f. 2.

2. P'Terotraches, Forsk. n. 332. Tentacula none; eyes 2; shcll nonc.

$$
\text { * Tail conical, keeled. }
$$

1. P. roronatit, Forsk. t. 158. f. 3, t. 156. f. 1; Philippi, t. 159. f. I; Chirje, t. 157. f. 1. P. aculeata, Forsk. t. 158. f. 5, t. 158. f. 1, 2. 'T'ail onls, linola Curiera, Perou \&- Lesueur, t. 160. f. 4; Blainv. t. 155. f. 7. F. Peronia, Peron \&. Lesueur, t. 160. f. 8. F. gibbosa, Peron \&. Jesueur, t. 160. f. 2. F. Forskalia, Peron \&Lesweur, t. 160. f. 3. F. junor, Quoy, t. 62. f. 6, t. 156. f. 2.

2. P. Frederica, Lesuenr, t. 160. 1'. 5; Chiaje, t. 157. f. 2 .

3. P. Ilippocampus, Philippi, t. 155. f. 5.

3*. P. mutica, Lesueur, t. 160. f. 1. P. hyalina, Forsk. t. 55. f. 2.

4. P. pulmonita, Forsk. t. 158 . f. 4.

5. P. Elwardsii, Desh. t. 15\%. f. 2.

6. P. luyalina, Chirije, t. 157 . f. 3 , var. preceding?

7. P. Lesucuri, Risso, t. 15\%, f. 4, var. precelling?

8. P. Kerandrenii. Firola K., Eydoux, t. 238. f. 2.

9. P. Quorana. Firola Q., D’Orb. t. 62. f. 1.

9*. 1. — Firole rouse), Toy. Uran. t. 87. f. 2.

** Tail short. Firoloilla.

10. P. neuleatis. Firoloisla a., Lesueur, t. 155. f. 9.

11. P. Blainvilleama. Firoloida B., Lesueur, t. 155. f. 1.

12. P. Desmarestia. Firoloila D., Lesueur, t. 155. f. \&; Eydoux, t. 233 . f. 1.

13. 1'. Eydouxii. Firole de Lesueur, Eydoux, t. 238. f. 3.

14. P. - Hooker, t. 155. f. 3.

3. Cerophors, n. 333. Tentacula 2, elongate; eves 2 ; shell nonc.

1. C. Lesucurii, D'Orb. t. 62 . f. 4.

2. C. Gaimarlii, D'Orb. t. 62. f. 3, not cop.

4. Cardisons, n. 334 . Tentacula 2 ; sbell cartilaginous, regularly involute.

1. C. pedimculata, D'Orb. t. 62 . f. 5 .

2. C. carinata, D'Orb. t. 62. f. 7 .

3. C. candina. Firola c., Rang, t. 155. f. 8. Carmairoille c., Eydow $x$, t. 239. f. 3 ; ? Hooker, t. 155. f. 4 ?

4. C. placenta. Carinairoile p., Eydoux, t. 239. f. 1. -

5. C. - ? ? ? t. 156 . f. 3 .

5. Carisaria, n. 335. Tentacula 2; shell glassy, thin.

1. C. mediterranea, Perou, Blainv. t. 633. f. 1. Pterotrachea Lophyra, Chiaje, t. 161. f. 2, t. 63. f. 3. C. crmbimm, Desh. t. 161. f. 1. C. vitrea, Costa, t. 154. f. 2 . l'terotrachea, Cuv. t. 159. f. 3, without shell.

2. C. Gaudichandi, Eydoux, t. 239) f. 4.

3. C. punctata, D'Orb. Amér. Mérirl. t. 11. f. 3, 4.

4. C. anstıali;, Qnoy \& Gaim. t. 62. f. 1.

5. C. 12. s., Adams, t. 239. f. 2. 


Fam. 9. Atlantida, 1. 148.

Body spiral, inclosed in a shell. Operculum calcareous.

1. Oxygyrus, 11. 174. Shell eartilaginous, keeled; nuclens involute, suleated, chalky; operculum amnular.

1. O. Keraudrenii. Atlanta K., Rang, t. 117. f. 3, operculum oblong kidney-shaped; D'Orb.t. 129. f. 2, t. 117. f. 1, opereulum triangular, broad; Lesueur, t. 117 c. f. 3; Eydoux, t. 240. f. 2. Ladas Keraudrenii, Cantr. t. 117 c. f. 6.

2. O. Rangii. Atlanta Rangii, Eydoux, t. 240. f. 1 \& 4, operculum roundish trigonsl.

2. Atranta, n. 175. Shell shelly, transparent, keeled; nucleus spiral, smooth; opereulum spiral; last whorl very large.

1. A. Peronii, Lesueur \&. Blainv. t. 117 c. f. 1, not correct; Rang. t. 117 b. f. 5 (onereulum incorrect); D'Orb. t. 117 b. f. $2 \& 6$; Eydoux, t. 240. f. 6. A. Kerandrenii, Quoy, t. 117 b. f. 1. A. Bivone, Pirajno.

2. A. Lamanoni, Eydoux, t. 240. f. 3.

3. A. inclinata, Eydoux, t. 241 . f. 1.

4. A. brumuea, Eydoux, t. 242. f. 5.

5. A. inflata, Eydoux, t. 24l. f. 3.

6. A. Gandichaudi, Eydoux, t. 241. f. 4.

7. A. Lesnemii, Eydoux, t. 241 . f. 6.

8. A. - - (A. bossue), Eydoux, t. 240. f. 5.

9. A. depressa, Eydoux, t. 242 . f. 4.

10. A. Quoyii, Eydoux, t. 242. f. 1.

11. A. rosea, Eydoux, t. 241 . f. 2.

12. A. helicinoidea, Eydoux, t. 24:2. f. 2.

13. A. involuta, Eydoux, t. 242. f. 3.

14. A. turriculata, D'Orb. ; Eydoux, t. 242. f. 6.

15. A. —, n. s., no. 1, Hooker, t. 117 c. f. 7 ; no. 2, Hooker, t. 117 c. f. 2 .

3. Steira, 11. 176. 1. St. Lamanoni, Eschsch. Isis, 1825, 734, t. 5. f. 3. Most probably a badly observed Atlanta.

b. Foot small, flat, with a resicnlar appendage on the hinder part of the under side. Body and shell spiral. Teeth lateral, mumerous, uniform, like Bullida. Eyes none. Tentacula bifid. Hermaphrodite, self-impregnating?

Fam. 10. Ianthinille, p. 148.

Perhaps should be placed in the preeeding Order.

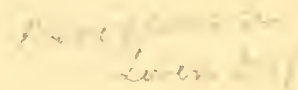

1. IANTHINA, n. 173.

1. I. vulgaris, Lister, t. 48. f. 1; Forstael, t. 48. f. 5, 6, 7 ; Curier, t. 117 a. f. 2; Lesueur, t. 117 r. f. 4 ; Quoy, t. 48. f. 2.

2. 1. nana, Qun!, t. 48. f. :3, 4. 


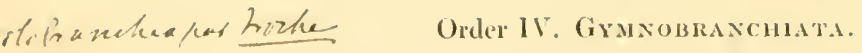

Gill exposed or eontractile into earities on the surface of the mantle. Adult animal without any shell. Larva shell-boaring, furnished with decidnous ceplnalie fins. Animal hermaphrodite, with reciprocal impregnation.

Fan. 1. Doridide $=$ Doridle, p. $16 \%$.

Gills surrounding the rent on the middle of the hinder part of the back.

A. Body convex; mantle convex, covering head and foot, simple. Doridina.

a. Gills retractile into a common cavity. Tentacula dorsal.

1. Glossodoris, n. 341. Gills strap-shaped, retractile.

etho rewoflersu moumen ras

1. G. verrueosa. Doris v., Cuvier, t. 65. f. 1 ; Chicije, t. 236 . f. 1.

2. G. Bertholeti. Dorisoligitata 1., D'Orb. t. 218. f. 1.

3. G. D'Orbignii, $D^{\prime} O r b$. t. 216 . f. 2 .

2. Actinodoris, n. 342. Gills strap-shaped, cut or forked at the tip, retraetile into a eommon eavity.

1. A. flammulata. Doris fl., Quoy, t. 234. f. 2.

2. A. seabra. Doris s., Quoy, t. 66. f.l.

3. A. eluenta. Doris c., Quoy, t. 66i. f. 3, 4.

4. A. maculosit. Doris m., Qunoy, t. 66. f. 5.

5. A. funosa. Doris f., Quoy, t. 2l6. f. 1.

6. A. sordida. Doris s., Quoy, t. 213. f. 3.

7. A. pumetata. Doris p., Quoy, t. (j). f. 5.

8. A. Mauritiana. Doris in., Quoy, t. 234. f. 1.

9. А. Krusensternii, Tilesius, t. 230, f. 5.

10. A. 'Tilesii, Tilesius, t. 2:30. f. 4.

11. A. tubereulosa. Doris t., Quoy, t. 65. f. 4.

3. Asteronotus, n. 347 .

1. A. cmenta. Doris e., Alder, MSS. t. 226. f.2. Torres Straits.

4. DeNDRodoris. Gills large, 4- or 5- lobed ; lobes doubly pillnate; rent ratlere behind the gills.

1. D. tuberenlata. Doris t., Cuvier, t. 6s. f. 1; Philippi, t. 231.f.11; Savigny, t. 233. f. 1; Rapp, t. 22). f. 6; Jolnston, t. 68. f. 8 .

2. D. limbata. Doris 1., Cuvier, t. 68. f. 3; Chiaje, t. 236. f. 4. D. viresrens, Risso, t. 67. f. $13,-$ ? t. 236 , f. 6.

3. D. setigera. Doris s., Rupp, t. 229. f. 5.

4. 1. glandifera. Doris g., Rapp, t. 229. f. 8; Chiaje, t. 236. f. 5.

5. Dokis, 11. 344. Gills arborescent, retractile; vent in centre of gills.

1. D. Argo, Blainville, t. 64. f. 12, from Savigny, t. 65. f. 2, t. 233.

f. 3. D. Argus, Rapp, t. 22: f. 7.

2. D. Jolnstonii, Alder \& Hanc. t. 215. f. 1. D. obvelata, Johnston, t. $67 . \mathrm{f} .4$.

3. 1). obvelata, Wiiller, t. 67. f. 7. D. repanda, Alder \& Hanc. t. $225 . \mathrm{f} . \mathrm{l}$.

1. D. flammea, Alder \&-Hanc. t. 215. f. 3. 


5. D. planata, Alder \& Hanc. t. 225. f. 2.

6. D. testurlinaria, Rissn, t. 68. f. 2; Chiaje, t. 23. f. 2 .

7. D. Semcle, Blainville, t. 69. f. 8 .

8. D. punctata, Riippell, t. 67 . f. 7 .

9. D. fusca, Mïller, t. 67. f. 15 . a onehedons

10. D. Flemingii, Forbes, t. 67. f. 11.

11. D. aurea, Quoy, t. 230.f.

12. D. violacea, Quoy, t. 224. f. 1.

13. D. venosa. Quoy, t. 61. f. 8.

14. D. impudica, Quoy, t. 64. f. 13.

15. D. limacina, Quoy, t. 64. f. 6. Gill in middle of back.

16. D. tomentosa, Philippi, t. 231. f. 8 .

17. D. lavis, Miiller, t. 69. f, 6; Thienemunn, MSS. t. 214. f. 6. = acanthatons

18. D. subleris, Thompson, t. 231. f. ?.

19. D. sparsa, Alder \&. Hanc. t. 27 i. f. = cuchudons

20. D. coccinea, Alder \&. Hanc. t. 271 . f. 1 .

21. D. nigricans, Rapp, t. 229. f. 1.

22. D. - Rïppell, t. 230 . f. 1 .

23. D. albolimbata, Riippell, t. 213, f. 2.

24. D. tenera, Costa, t. 214 . f. 5 .

25. D. luteo-rosea, Rapp, t. 22?. f. 4.

26. D. punctata, D'Orb. t. 218 . f. 2.

27. D. marginata, Montag. t. 223. f. 3. - foncoson

28. D. —- Savigny, Egypt, t. 233. f. 1.

29. D. Incii, Alder, IISS. t. 226 . f. 1 . a actend. fumera no $5 / 102$

30. D. candida, Rapp, t. 269. f. 4. Gill in midelle of back.

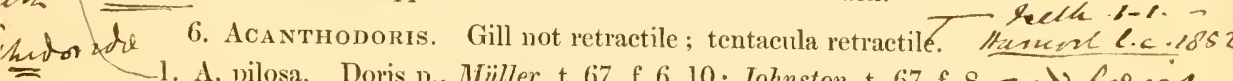

1. A. pilosa. Doris p., Müller, t. 67. f. 6, 10; Johnston, t. 67. f. 8. zad Cars

7. Ckratodoris. Tentacula elongate, filiform, not retractile.

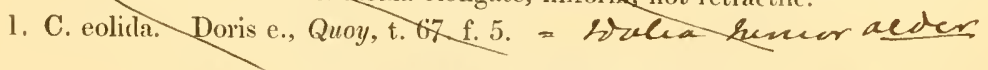

b. Gills, each retractile into its proper cavity.heneer la. Zahenles

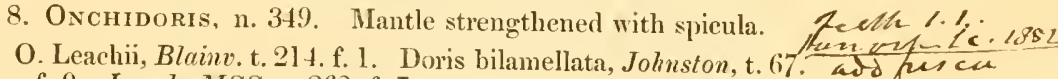

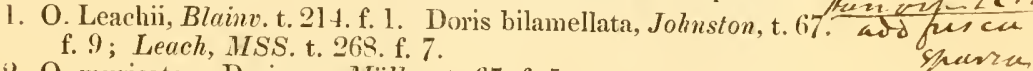

2. O. muricata. Doris m., Miiller, t. 67. f. 5.

3. O. diaphana. Doris đ., Alder \&.Hanc. t. 217 . f. 1.

4. O. pusilla. Doris p., Alder \&. Hanc. t. 217 . f. 2 .

5. O. sanguinea. Doris s., D'Orb. t. 223. f. 5. D. mbira, D'Orb. fee retroule t.

6. O. - - Doris, Savig. t. 233. f. 4.

9. Villiersia, n. 350. Mantle with a continuous calcareous shield, pierced with holes for the tentacula, gills and rent.

1. V. scutigera, D'Orb. t. 213. f. 1. Chasule inonems acder

10. Ilexabranchus, n. 348. Gill tree-like, in a ring; labial fecler dilater, broal, crenate.

1. 11. pretextus, Ehrenb. t. 237.

2. H. sanguineus. Doris s.. Riipyell, t. 68. f. 1. 
3. H. laciniatus. Doris 1., Cuvier, t. 230. f. 2, copied Blainv. t. 65. f. 3 .

4. II. Marrinalis. Doris $\mathrm{m}$. Q Qnoy, t. 221. f. 2, t. 224. f. 2.

5. II. sandwichensis. Doris s., (2uoy, t. 235.

6. II. Adansii, Allams, MS'S. t. 2I9. f. I.

11. IIferta braxculs, fdams, 1819. Gills tree-like, in a broad lumate series.

I. II. Bumettii, Adams, t. 21!) f. :2.

12. Atagema. Gills very small, at the end of a dorsil sac.

1. A. carinata. Doris c., Quoy, t. 64.f. 11.

в. Body angular; mantle distinct, simple, not cocering the head and foot.

13. Goniodoris, Forbes, 345, pars. Tentacula dorsal, not retractile. Gill lamreolate, pimate.

1. G. nodosa, Alder \&. Hanc. t. 227. f. 1. G. elongata, Thompson, t. 231. f. 3 ; cmarginata, Forbes, t. 231. f. 5 . Doris, Leach, t. 268. f. 9. D. Barvicensis, Jolinst. t. 69. f. 10.

2. G. castanea, Alder \& Hanc. t. 22:2. f. I.

3. G. pulcherrima. Doris 1., Cantr. t. 232. f. 1.

4. G. villefranea. Doris v., Risso, t. 25:2. f. 2.

5. G. elegantuli. Doris e., Phitippi, t. 231. f. 7.

6. G. purpurea. Doris p., Laurillard, t. 2:34. f. 4.

7. G. picta. Doris elegans, Cantr. t. 213. f. 4; Philippi, t. 231 . f. 17.

8. G. crmulea. D. gracilis, Rupp, t. 229. f. 2. D. tricolor, Cant?. t. 231 . f. 10.

9. G. albescens. Doris palens, Rapp, t. 229. f. 3.

10. G. marmorata, Savigny, t. 64. f. 14.

11. G. Webbii. Polveera Webbii, D'Orl, t. 2'32. f. 5.

12. G. impulica. Doris i., Riippell, t. 23:2. f. 4.

13. G. tinctorum. Doris t., Riippell, t. 69. f. 5.

14. G. obsoleta. Doris o., Riuppell, t. 69. f. 4.

15. G. pallida. Doris p., Riippell, t. 69. f. 3 .

16. G. infuseata. Doris i., Riüppell, t. 6.9. f. 2.

17. G. pulchella. Doris p., Riippell, t. 66. f. '2.

18. G. atromarginata. Doris a., Cuvier, t. 64 . f. 10, t. 180. f. : Quoy, t. 64. f. 4 .

19. G. lemmiscata. Doris 1., Quoy, t. 2.23. f. 6.

20. G. magnifica. Doris m., Quoy, t. 64. f. 2 ; Banks, t. 67. f. 17.

21. G. reticulat:. Doris r., Quoy, t. 61. f. 7.

22. G. elegans. Doris e., Quoy, t. 64. f. 9.

23. G. lineata. Doris rayée, Eydour, Yoy. la Bonite, t. 2:20. f. 4.

13*. Brachychlanis, n. 315. Tentacle in frout of mantle. B. pantluerina, Ehrenb.

c. Borly subangular; mantle distinet, fringed with tentacula. Triopina.

1.4. Triopa, 11, 352. Forehead papillose; teeth, central none. lateral 8.8.

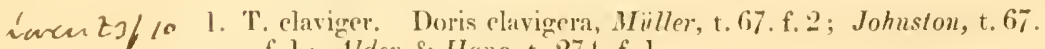
f. 1 ; Alder \&. Hanc. t. 27 t. f. 1 . 
I hum lately ben neconsidenewes the

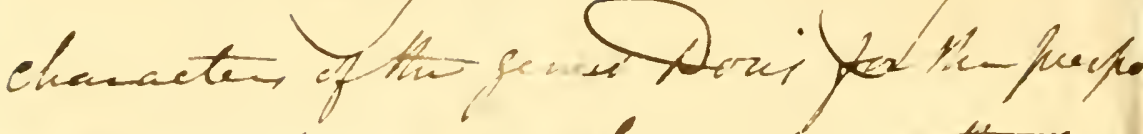

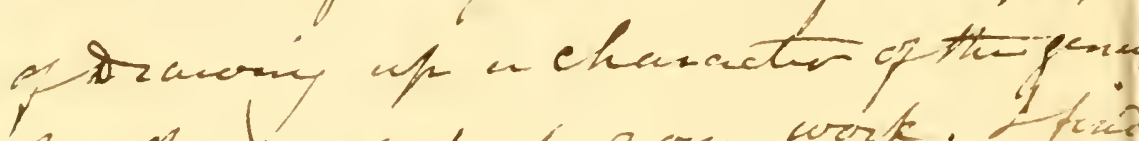
for. An neat peut of one work.

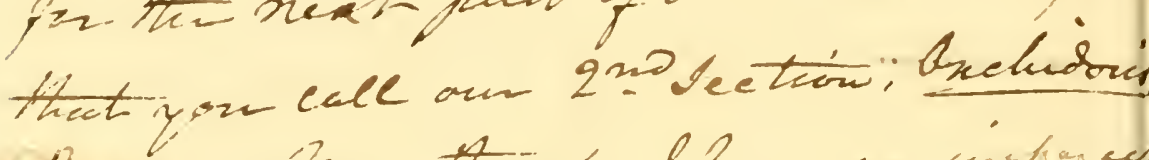

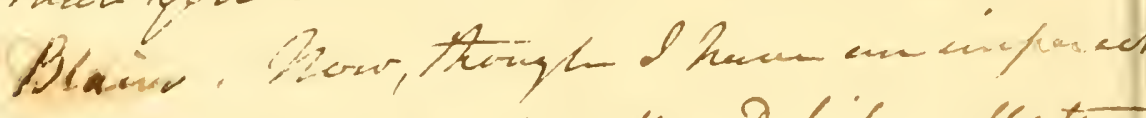
reellection of deaing the D. Gilcunallente

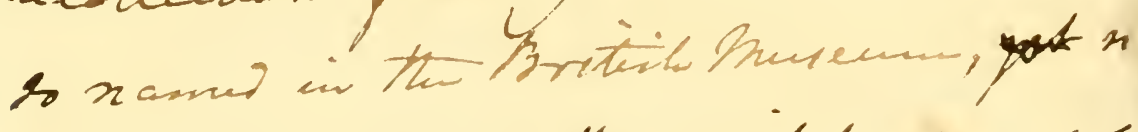

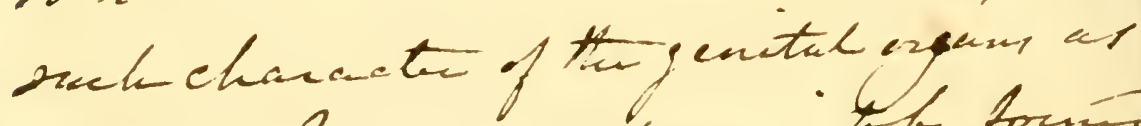

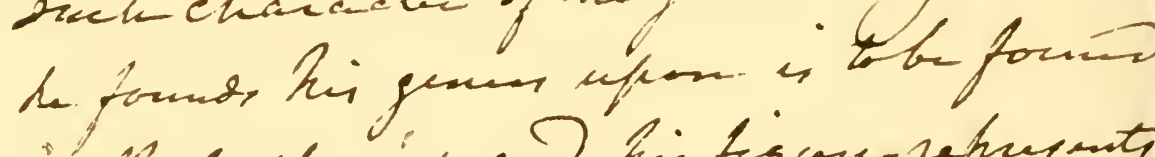

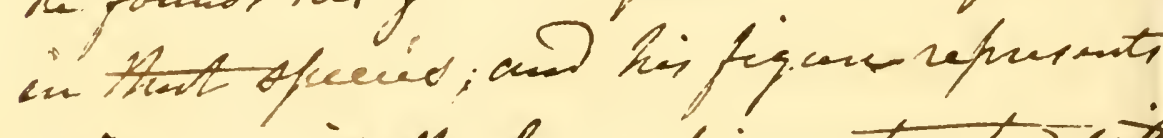

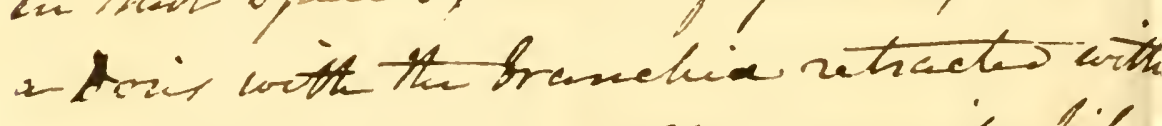
a cosicty, whide is ast fle car with bitan

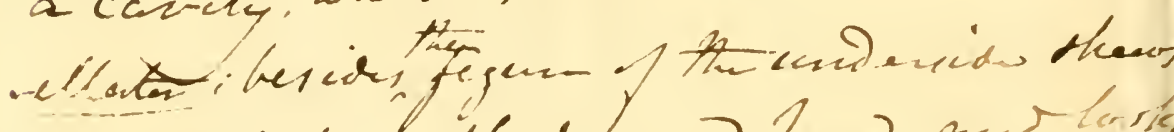

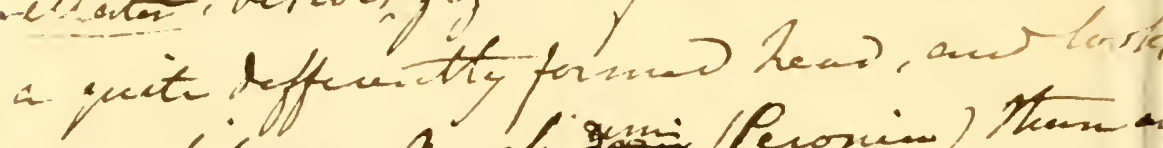

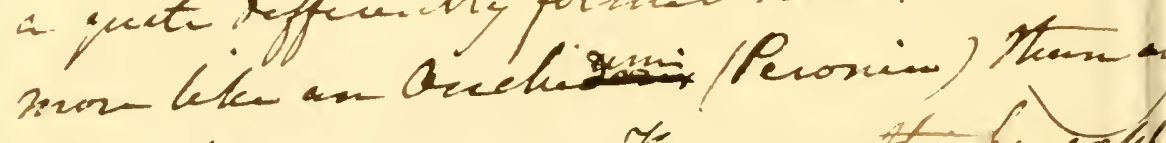

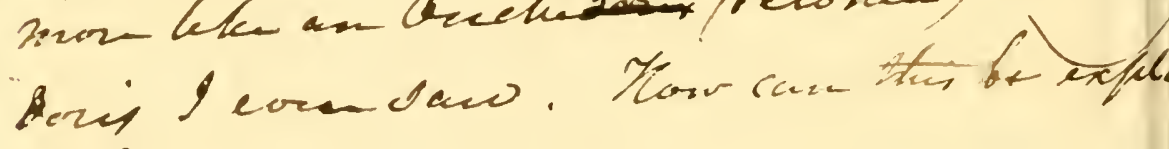
a) 2

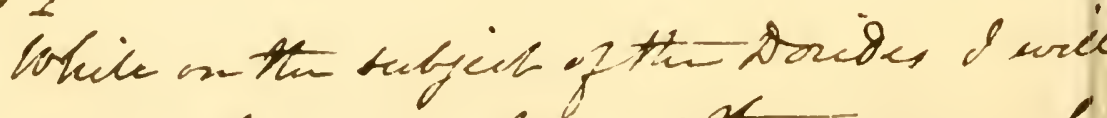
ventene to make tevo in thes semacts

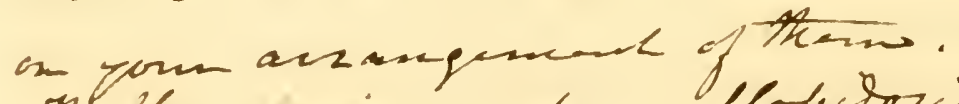

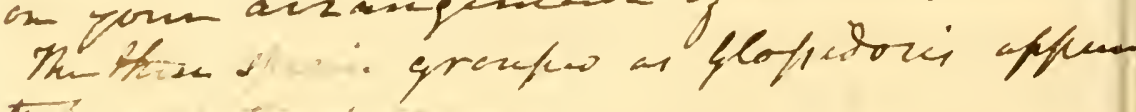

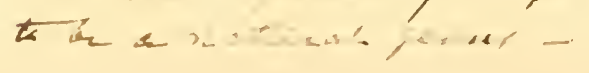


Aretenosoni costemis mon them on tafer, and thow whith come mosks atumally into

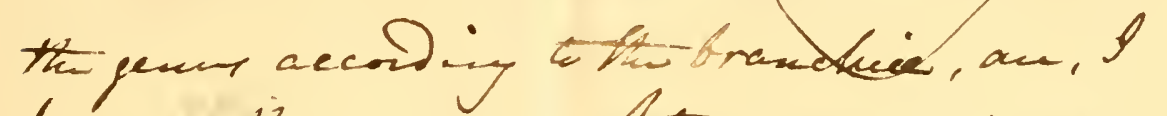

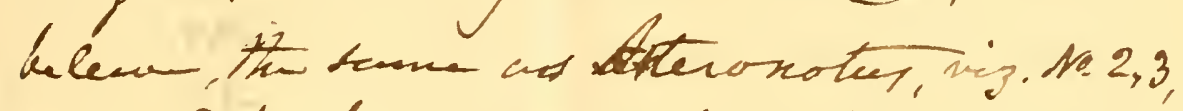

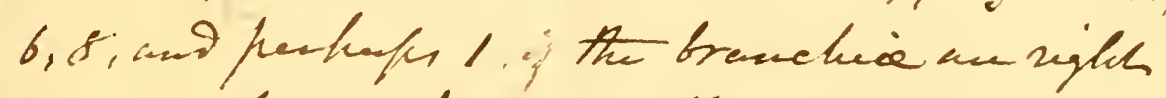
Prano but it has mon the afpecanenen of in Hexabranelues. Mhe others \& theile bo not

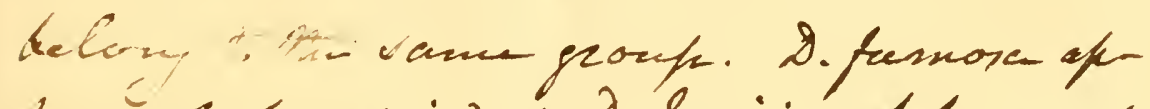

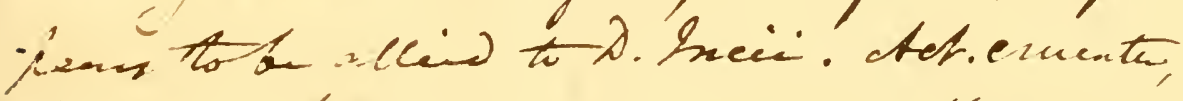

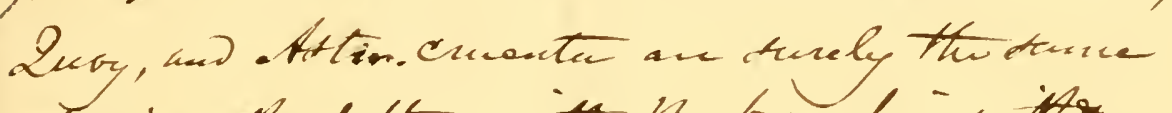

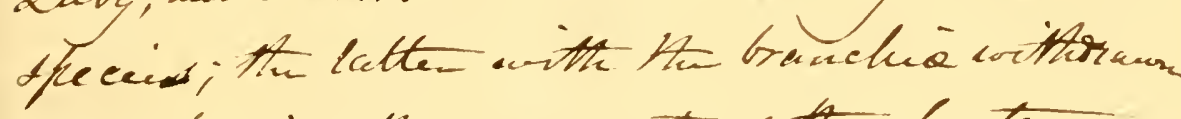

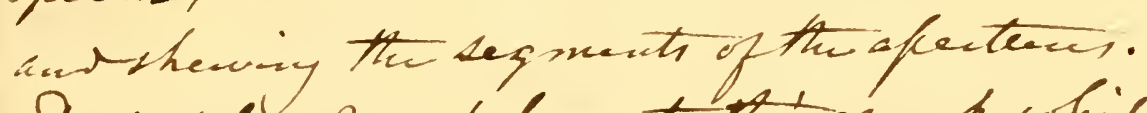
Driir tolere, bev. belongs to this grouk whil

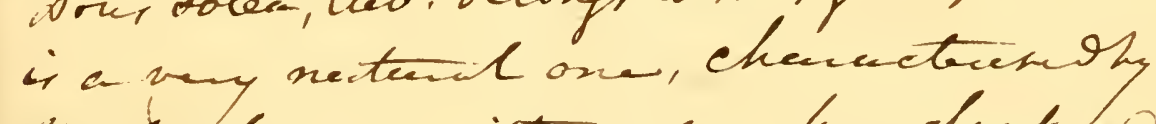

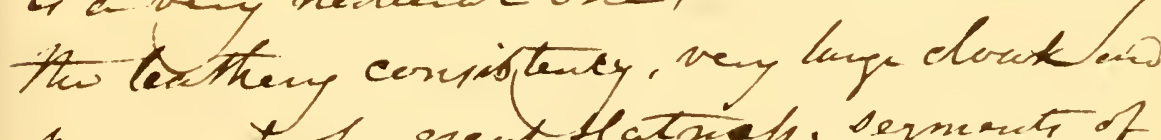

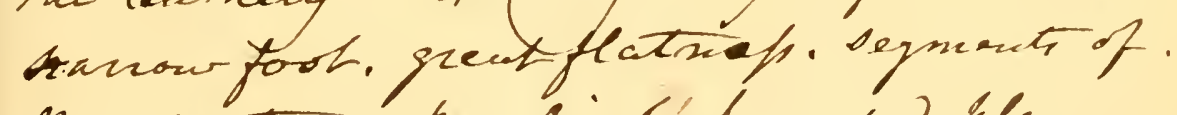

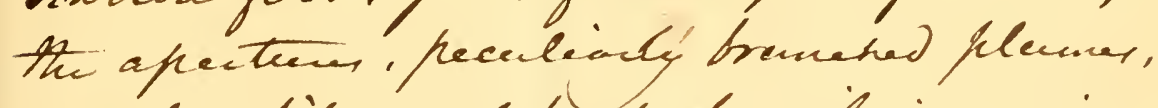
cut leaflike orcal tertader. In i cunsines ales thint moth of tirm ar bloteh, with colone on the rendur tien of the clonkIobrewn you huve nat asples Pterodon's of Phrenbery, in which I think yor-are right,

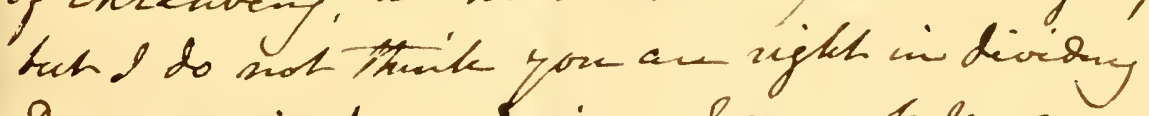
sursobir from Socis as 8 cannot bee ony retuidtion on the porition of the vent -

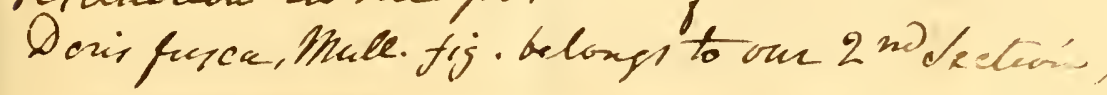




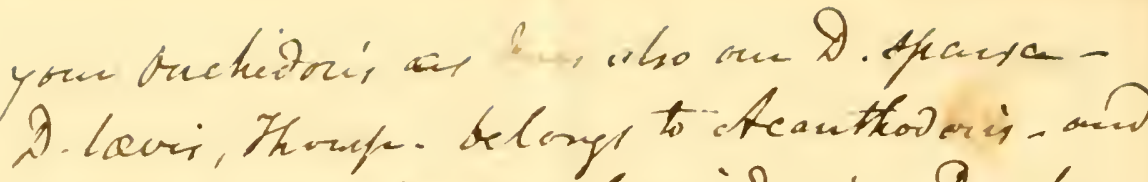

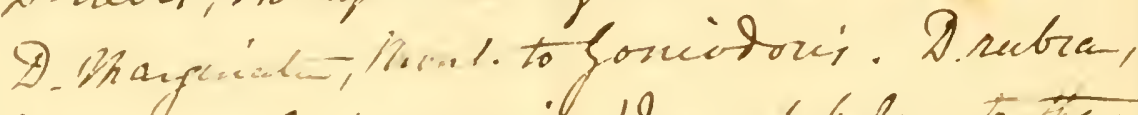

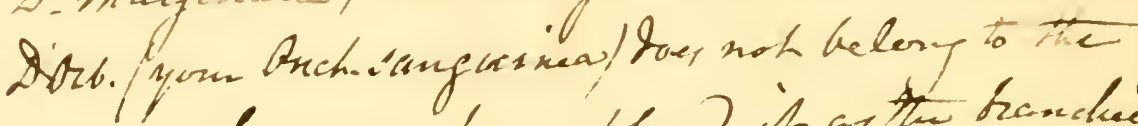

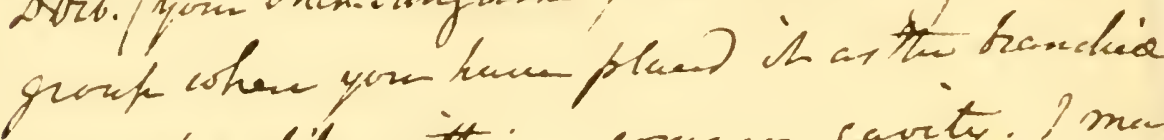

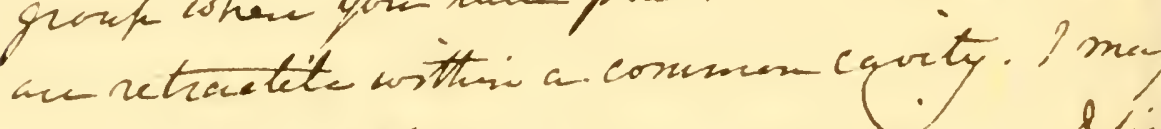
inenteon hen that we vem wrong own difin

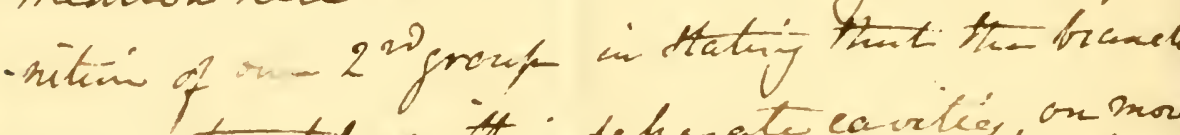
wer vetrastil avitusi defresete cavilie, on mon

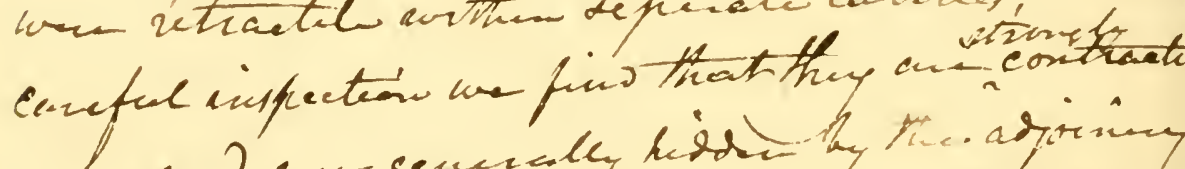

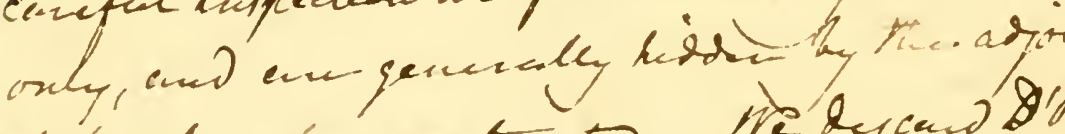

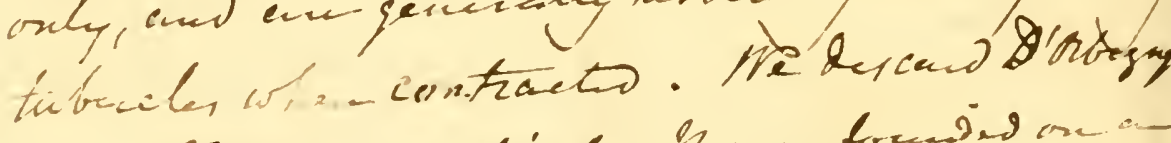

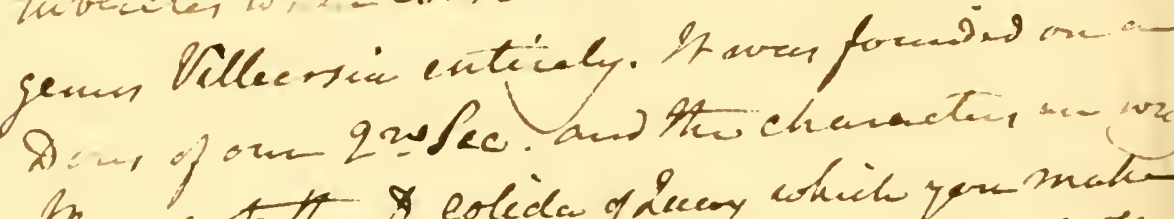

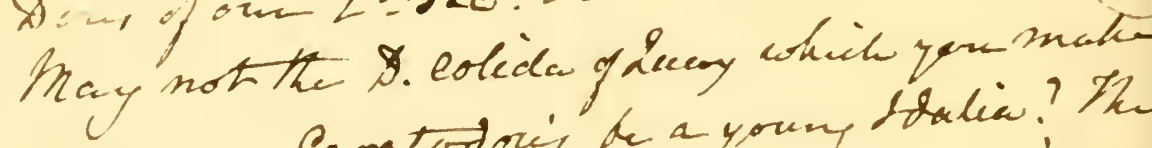

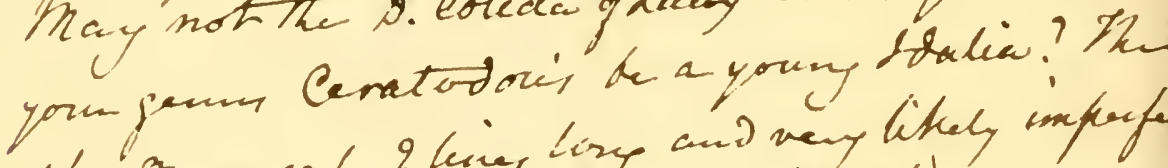

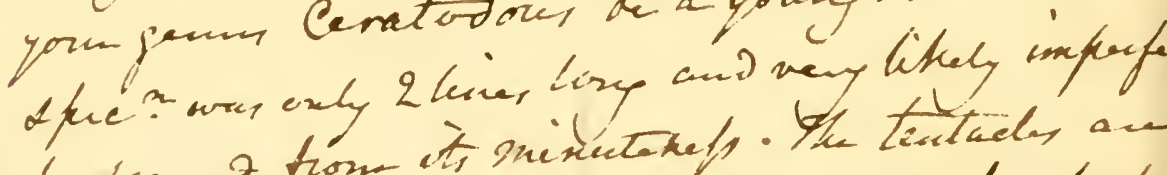

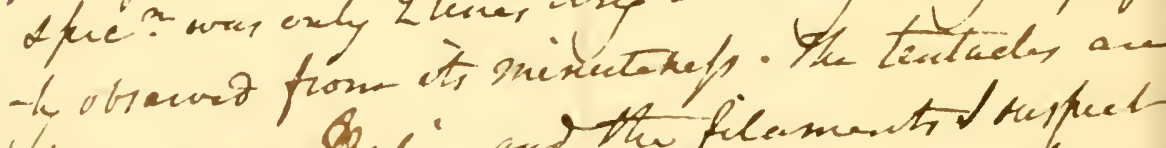

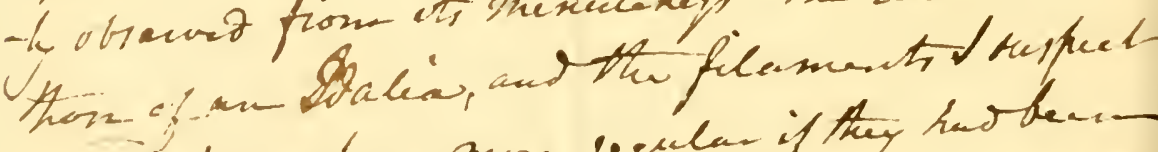
i) have hem mone ugular if they hosthen-

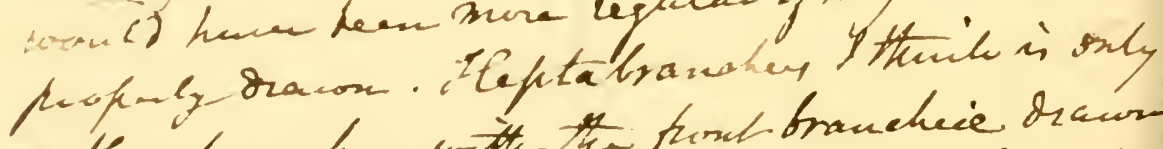
a Hexatrenchers with the pund brancheie or awn

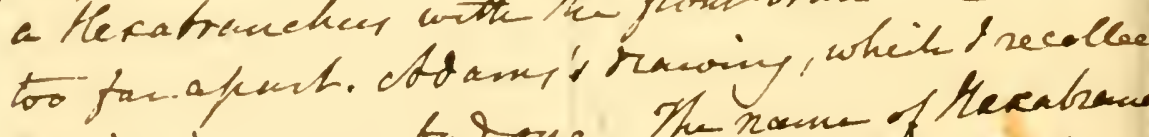
deany, it a evey bas one. The nown of Hexabane

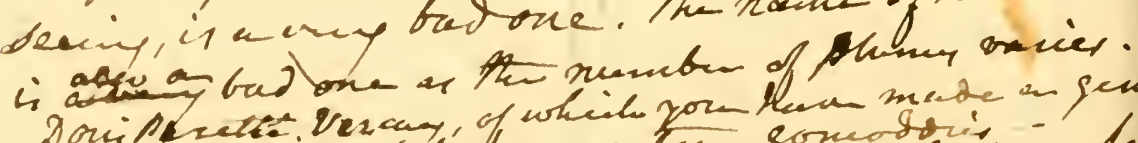

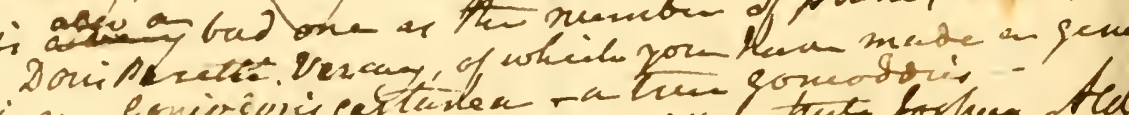

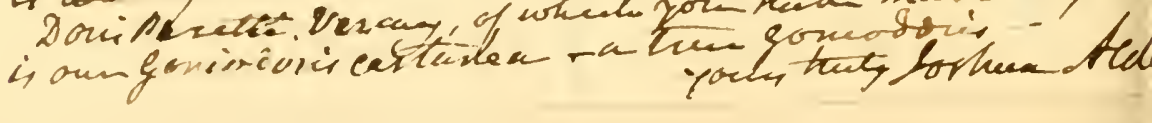






15. Ina lia, n. 356. Foreherd simple, rounded; teeth, eentral 1, Laven C 36/6 lateral $2 \cdot 2$.

l. I. elegans, Leuck. t. 199. f. 10.

2. I. cirrigera, Philippi, t. 231. f. 1. Euplocamus c., Philippi, t. 231. Lever \& \&f" f. 4. I. aspera, Alder \& Hanc. t. 215 . f. 2.

3. I. quadricomis. Doris q., Nont. t. 223. f. 2.

4. I. plumosa. Euplocamus p., Thompson, t. 231. f. 2.

5. I. dubia. Polycera d., Sars, t. 232. f. 6.

6. I. Lessonii. Polycera L., D'Orb. t.204, f.9; Alder \& Hanc.t.274. f. 3 .

7. I. crocea. Euplocamus c., Philippi, t. 67. f. 3. Doris ramosa, Cantraine, t. 231. f. 15.

8. I. frondosa. Euplocamus f., Philippi, t. 231. f. I.

9. I. laemosa. Euplocamus 1., Philippi, t. 231. f. 6.

\section{Axcula, 11. 357. Gills pimate.}

1. A. cristata. Miranda c., Alder \& Hanc. t. 222. f. 2. Polycera c., Alder \&; Hanc. t. 204. f. 4.

D. Body elongate, subangular; muntle indistinct. Policerina.

a. Forehead papillose.

17. FGiRes, 11. 353. 'Teeth, central none, lateral $17 \cdot 17$.

1. A. punctilucens, Alder \& Hanc. t. 274. f. 2. Polyeera p., D'Orb. t. 204 . f. 9.

2. A. maura. Doris manra, Forbes, t. 231. f. 12.

3. A. Lessonii. Polycera L., D'Orb. t. 204. f. 9.

18. THeCACERA, n. 354 .

1. T. pennigera. Doris p., Montag. t. 236. f. 3.

19. Polycera, 1. 355. Frontal appendage simple.

1. P. quadrilineata. Doris q., Müller, t. 64. f. 3. D. flava, Montag. t. 223. f. 1. Var. 1. Alder, t. 204. f. 1. Var. 2. Alder, t. 204.

f. 3; Thompson, t. 205 . f. 5 .

2. P. lineata, Risso, t. 232 . f. 3.

3. P. typica, Thompson, t. 204. f. 2.

4. P. comuta. Doris c., Niuller, t. 64. f. I.

5. P. ocellata, Alder \& Hanc. t. 227 . f. 2 .

6. P. ornata, D'Orb. t. 204 . f. 8.

7. P. citrina, Alder, t. 204. f. 6.

20. Plocaloceros, n. 359. Frontal appendage branched.

1. P. oeellatus, Riippell, t. 69. f. 1.

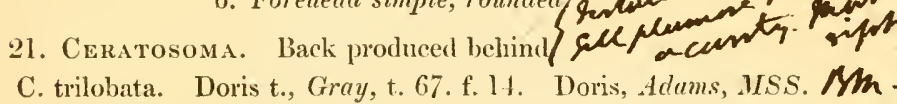


Fan. 2. Tritoniade, p. 16i.

Gill on side of the back, lamellar, papillary or brancherl. 'Tectl, central 1, lateral $10 \cdot 10$ to $24 \cdot 24$. Tentacula 2, retractile withiu sheath.

a. Stomach simple. Tritoniana.

1. Tritonia, 11. 362. Teeth, eentral 1, lateral $10 \cdot 10$ or $14 \cdot 14$.

* Frontal tentarula tufted.

1. 'T. Homberwii, Curier, t. 212. f. 5, 9; Blainv. t. 212. f. 3; Johnston, t. 211 . f. 2.

2. 'T', aeuminata, Costa, t. 2): 2. f. 8.

3. T. quadrilatera, Philippi, t. 21:2. t'. 7 .

4. 'Т. decaphylla, Cantraine, t. 198. f. 11.

5. T. Costa, Jeramy, t. 209\%. f. 33.

6. T. rubra, Riippell, t. 2l2. f. 3.

7. 'T. glama, Rüppell, t. 211. f. 4; Savigny, t. 22-7. f. 1, 2.

R. 'T. eranolumeliata, Rüppell, t. 211. f. 3 .

9. 'T. 'Thethydea, Chiaje, t. 2ll. f. 1.

** Frontal tentacula simple. Candiclla.

10. 'T. plebeia, Johuston, t. 212. f. 4; Alder \& Hancock, t. 2019. f. 2.

b. Stomach branched. Melibeina.

2. 2. Dexpronotus, 11.364. Gill in single series; teeth, eeutral 1, lateral $10 \cdot 10$.

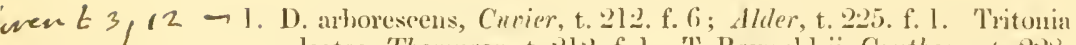
lartea, Thompson, t. 212. f. 1. 'T'. Reynoldsii, Couthouy, t. 2.28. f. 2; 'Thienemam, MSS. t. 21- f. 4 .

3. Doтo, 11. 365. Gill clarate, compoumel.

1. D. furgilis, Melibiea f., Forbes, t. 198. f. 7 .

2. D. coronata, Alder \& Hanc. t. 208. f. 2. Melibea ('., Johnston.

10 t. 198. f. 1. 'Tergipes e., D'Orb. t. 199. f. 8 (Voy. Bonite, t.2 1*, arat.).

3. D. pimatifida. Dorjs p., Montayne, t. 198. f. 5.

4. D. maculata. Doris m., Montag. t. 198. f. 3.

4. Gellina. Gill clavate, simple.

1. G. aftinis. T'ergipes a., D’Orb, t. 199. f. 9.

2.0.12 - Lomanotes, 11. 366. Gill erest-like.

1. L. Genei, I'erumy, t. 2.21. f. :2.

2. I. masmoüitus. limmenis marmorata, Alder \& Hanc. t. 221 f. I.

6. ScrLlasa, n. 368. Teetl, central 1, lateral 21-21.

1. S. pelagrea, Blaine. t. 20)3. f. 1 ; Cmier, t. 20:3. 1'. 3.

2. S. ghomiforlensis, Forskael, t. 20(13. f. 4 .

3. S. Qmoyii. S. ghomforlensis, Qmoy, t. 2013. f. 5.

4. S. IIookeri, Gray: Ionker's MSS. t. 2(1);3. f. 6.

7. ? NEREA, 1. 36?\%

1. X. punctata, Lesson, t. 2013. 1.2. 

$1=$

$146 \quad \cdots$ 
8. TETHYS, n, 370 .

1. T. leporina, Chiaje, t. 210. f. 1 ; Deshayes, t. 2010. f. 2.

9. Bornella. Back three tufts of simple and branched tentacula.

1. B. Adamsii, Gray; Adams, MSS. t. 176. f. 6.

Fam. 3. Glaucidre.

Gill on side of lack, papillary. Tentacula 2, linear, without sheaths, rarely wanting. 'Teeth, central 1 , no lateral.

a. Anus lateral; gills papillose, in groups. Glancina.

1. Glaucus, n. 371=Pleuropus, Rafin. 1815.

1. G. radiatus.

2. G. atlanticus, Blainv. t. 20.2. f. 4 ; Curier, t. 201 . f. 3.

3. G. Draco, Eschsch. t. 202. f. 6.

4. G. Forsteri, Quoy, t. 2(02. f. 3.

5. G. Eucharis, Peron \&- Lesueur, t. 201. f. 1, 6; Voy. Bonite, t. 24, rantor seckitury t2t. $24 *$, anat.

6. G. pacificus, Esch. t. 202. f. 1. G. distichoieus, D'Orb. t. 202. f. 7; Dr. Sinclair, MSS.t. 202. f. 2; Dr. Hooker, MSS. t. 201. f. 4 .

2. LANiogerus, n. 37\%.

1. L. Elfortii, Blainv. t. 231. f. 13. Probably only a Glaucus which has lost its gills ; t. 201. f. 2 \& 5 from Dr. J. Hooker's MSS. are probably the same.

b. Vent lateral. Gills in rows on each side. Eolidina.

3. Eolid1A, n. $373=$ Psiloceros, Menke, 1844= Imphorina, n. 395, junior. Gills in longitudinal series; tentacula elongate, subulate; labial feelers elongate, subulate.

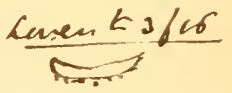

\footnotetext{
2 2ut $\quad *$ Front of foot ungular.
}

1. E. papillosa, Johnston, t. 197. f. 3. Doris p., Baster. t. 197. f. 6; Montag. t. 195. f. 43; Leach, t. 268. f. 8.

2. E. limacina. Eolis l., Philippi, t. 194. f. 2.

3. E. Scacchiana. Lolis S., Philippi, t. 194. f. 5.

4. E. Cuvieri, Blainv t. 195. f. 6.

5. E. ? äiversa. Eolis d., Couthouy, t. 196. f. 4.

6. E. salmonacea. Eolis s., Couthouy, t. 196. f. 5.

7. Eolidia —? t. 205. f. 8 .

8. E. Histrix, Otto, t. 205. f. 1.

** Foot subulate in front.

9. E. paradoxa. Eolidina 1., Quatref. t. 194. f. 7.

4. Montagua =Carolina, n. 375, not Gioenia. Tentacula and labial feelers subulate; gills in rather distant transierse rows.

* Front of foot produced, subulate.

1. M. peregrina, Cavolina, t. 205. f. 10. Doris p., Chirije, t. 194.

f. 9(?). Carolina p., Deshriyes, t. 198. f. 11.

2. II. Inngicornis, Doris l., Moutague, t. 195. f. 8. 
Loliclia a., Quny, t. 194. f. 3.

4. M. glauca. Eolis, Alder \& Hanc, t. 27:2. f. 1.
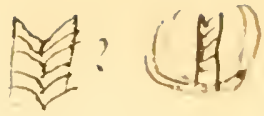

5. MI. viridis, Forbes, t. 199. f. 11.

6. M. angulata. Lolis a., Alder \& Hanc. t. 200 . f. 3.

. M. concimna. Eolis c., Alder \&- Hanc. t. 192. f. 1.

** Front of foot subangular.

8. M. nana. Eolis n., Alder \& Hanc, t. 272. f. 2.

9. M. olivacea. Eolis o., Alder \& Hanc. t. 192. f. 3, t. 197. f. 2.

10. M. cingulata. Eolis c., Alder \& Hanc, t. 207. f. 2.

11. M. purpuraseens. Eolida p., Fleming, t. 19\&. f. 10.

12. M. corulea, Doris c., Hontague, t. 195. f. 2.

13. M. pallida. Eolis picta, Alder \&-IIanc. t. 207. f. 3.

14. M. amena. Lolis a., Alder s. Hanc. t. 206. f. 3.

15. M. arenicoli. Eolis a., Alder \&. IIanc. t. 207 . f. 1.

16. M. tricolor. Eubranchus t., Forbes, t. 198. f. 4. Eolis t., Alder \& Hanc. t. 193. f. '2.

17. M. Warmin. Eolis F., Alder \& Hanc. t. 195. f. 1. junior? Amphorina Alberti, Quatref. t. 199. f. 5.

18. II. rulra. Cavolina r., Cantraine, t. 194. f. 1.

19. M.? lottini. Eoliclia 1., Lesson, t. 194. f. 10.

20. MI. uatuns. Carolina n., J'Orb. t. 194. f. (i.

21. M.? pimuata. Eolidia p., Eschsch. t. 195. f. 3.

22. M. bella. Lolidia b., Riippell, t. 195. f. 7.

23. M.? gymota. Eolis $2 .$, Couthouy, t. 196. f. ?2.

24. M. - Eolide de Curier, Eydoux, t. 204. l" t (1roy. Bonite, t. $24 \mathrm{~A}$. B. (lmat.).

5. Pindaxa = Carolina, $D^{\circ}$ Orb. 'Tentacula clarate, perfoliate; labial feelers subulate; yill in cross rows.

* Foot rounded in front.

1. 1. patagonica. Carolina p., D'Orb. t. 191. f. 3.

2. P. Inca. Carolina I., D'Orb. t. 191. f. 1, 2, t. 191. f. \&.

3. P. longicanda. Eolidia l., Quoy. t. 194. f. 11 .

4. P. northumbrica, Eolis n., Alter \& Hanc, t. 20s. f. 1.

** Foot subulate in front.

5. P.? carulescens. Eolidia c., Laurillurd, t. 194. f. 4.

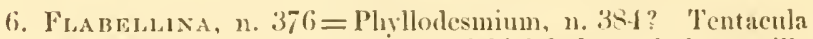
subulate, annulate or perfoliate; labial feclers subulate; gills clinstered.

* Front of foot subulate.

1. F. affinis, Curier, t. 198. f. 13.

2. F. punctata. Eolis p., Alder \&. Hunc. t. 206, f. 2.

3. F. peclata. Doris p., Montuy. t. 195. f. ?.

4. F. Drummondii. Lolis I)., Alder s. Hance t. 273. l'. 1.

5. F. Curieri. Eolis C., Johnston, t. 195. f. 1. Eoliclia, Curier. t. 195 . f. 10 .

6. l'. Iostoniensis. Lolis b., Couthouy, t. 196. f. 3.

** Front of foot subangular.

7. F. coronata, Eolis c., Alder \& Hanc. t. 191. f. 4, t. 206f. f. I.

8. 1'. annulicornis. Eolis a, Nat, Curs, t, 205, f. 5. 


9. I.? rufibranchialis. Eolis r., Aleter \& Hane. t. 273. f. 2. T'tacula nearly smooth.

10. F.? pellucila. Eolis p., Alder \& Hanc. t. 200. f. 1. 'Tentacula nearly smootly.

11. F.? Landsburgii. Eolis L., Alder \&. Hanc. t. 273. f. 33.

12. F. mimima. Limax m., Forsk. t. 205. f. 4.

13. F. crassicornis. Cavolina c., Eschsch. t. 195. f. 5.

14. F. subrosacea. Cavolina s., Eschsch. t. 195. f. 4.

7. Favorinus. Tentacula with a single subterminal fold; labial feelers elongate; gills in tufts; front of foot subulate.

1. F. albus. Lolis alba, Alder \&- Hanc. t. 192. f. 2.

8. Corypiella. Tentacula subulate, smooth; labial feelers subulate; gills in tufts; foot subangular in front.

1. C. Landsburgii. Eolis L., Alder \& Hanc. t. 273. f. 3.

2. C. rufobranchialis. Lolis r., Alder \& Hanc. t. 273, f. 2.

3. C. pellucida. Eolis 1., Alder \& Hanc. t. 200. f. 1 .

9. Tergipes, n. 377. Tentacula subulate, simple; labial feelers short; gills in a single lateral series; foot square in front.

1. T. laciniatus, Blainville, t.198. f. 2. Limax tergipes, Forsk. t. 205.

f. 3,5 .

2. T. Lamarckii, Desh. t. 198. f. 12.

3. T. desprectus. Eolis d., Alder \& Hanc. t. 193. f. 3.

4. T. - K Krusenstern, t. 205 . f. $2,7$.

5. T. adspersus, Nordmann, Mem. Acad. Petersb. t. 1. f. 3, 4.

(i. 'T. Eelwardsii, Nordmann, l. c. t. 1. f. 1, 2.

10. CALliopeA, n. 374. Gills in lateral series; tentacula none; labial feelers elongate, subulate; front of foot angular.

1. C. bellula, D'Orb. t. 194. f. 5 .

\section{c. I'ent dorsal.}

11. HermÆA, n. 378. Gills clavate, smooth.

1. H. bifida. Doris b., Montag. t. 198. f. 6.

2. H. dendritica, Alder \& Hanc. t. 272. f. 3; M. Edwards, t. 205. f. 9 .

12. Alderia, 11. 380. 1. A. modesta.

13. JAnus, n. $361=$ Antiopa, Alder \&. Hanc. Gill cyliudrical, KeTh so 1.4.0 simple, crowded; tentacles perfoliate.

1. J. Spinolx, Verany, t. 200. f. 2.

14. Struliger, n. 383. Gills fusiform, simple.

1. S. ornatus, Elırenb. t. 194. f. 4.

15. Proctonotus, n. $382=$ Venilia, Alder \& Hanc. Gills warty. - -0 b

1. P. mucroniferus, Alder \& Hanc. t. 197. f. 1.

2. P.? pilosus. Zephrina pilosa, Quatref. t. 199. f. 1.

Fam. 4. Phyllidiada, p. 168.

1. Phyllidia, n. 398.

1. P. pustulosa, Cuvier, t. 312. f. 1 ; Rüppell, t. 312. f. 5 .

2. P. occllata, Cuvier, t. 312. f. 8. 
3. P. trilineata, Curier, t. 312. f. 3, 6, 7; Blainv. t. 312. f. 4.

1. P. albo-nigra, Quoy, t. 312. f. 2.

2. IYPOBRANCIIAA, 11. 35I.

1. II. fusea, Adlams, MSS. t. 220 . f. 1.

Fam. 5. Diphyllidialle.

cemen L3/14 - 1. Diphyllidia, 11. 399. Teeth, central 1, lateral its, uniform, serrated.

1. D. lineata, Otto, t. 204. f. 1; Cantr. t. 205. f. 1; Desh.t. 20 5. f. 3. Pleurophyllidia neapolitama, Chiaje, t. 205. f. 2. Linguella Elfortii, Blaine. t. 205. f. 4.

2. D. veriucosa, Cantraine, t. 205. f. 6 .

3. D. ocellata, Deshayes, t. 205. f. 5.

4. D. pustulosa, Philippi, t. 204. f. 2.

Fam. 6. Phyllirshoide, p. 167.

1. Phyllirriö̈, n. 396 .

1. P. bucephala, Peron, t. 12.f. 4; Blainv.t. 15. f. 6; Joy. Bonite, t. 24, anat.

2. 1'. Lichtensteinii. Euryliee L., Eschsch. t. 133. f. 4.

3. l'. amboinensis, Quoy, t. 153. f. 3.

4. l'. rosemu, D'Orb. t. 153. f. 2.

5. l'. punctulatum, Quoy, t.j. Fi. f. 1.

6. P'. rubrum, Quoy, t. 153.f. 5.

Fan. 7. Placobranchida, 1) 16i.

Gills radiating lamina or vessels on the back.

T. Placobraxches, n. 387 .

1. P. ocellatus, Rang, t. 176. f. 4.

2. ELYSIA, n. 388 .

1. L. timida, Risso, t. 18. f. 8, 9; Philippi, t. 180. f. 1:2.

2. L. fusea, Philippi, t. 180. f. 10, 11 .

3. E. viridis, Cantr. t. 180. f. 14; J'erany, t. 176. f. 2. Aplysia r., Montagu, t. 146.f.4. Aeteon v., Sars, Bese. t. l8.f. 1-5. Aplysiopterus, Chiaje, t. 18. f. 7. Aeteon r., M.-Edwards, t. 199. f.2. $-?$ t. 176 . f. 5 .

4. E. clegans. Acteon e., M.-Eduards, t. 199. f. 4.

5. E. australis Deteon a., Quoy, t. 180.f. 6.

Fan. 8. Limapontiade, p. 167.

1. Limapontia, n. 390 =Chaliclis, n. 391.

1. L. nigra, Aller \& Hanc. t. 177. f. 3, 4.

2. L. corvulea. Chalidis c., Quatref. t. 199. f. 7.

2. Pelta, 11. 451.

1. P. coronata, Quatref. t. 199. f. 6.

3. ACTEONiA, n. 393.

1. A. corrugata, Alder \& Hanc. t. 177. f. 2.

2. A. senestra, Quatref. t. 199. f. 4. 

Ceycisduller Var

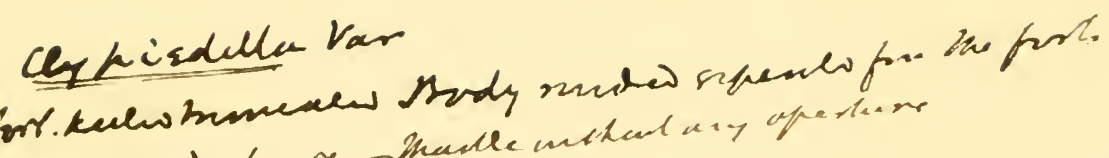

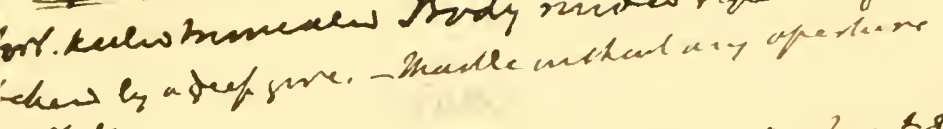
shale -

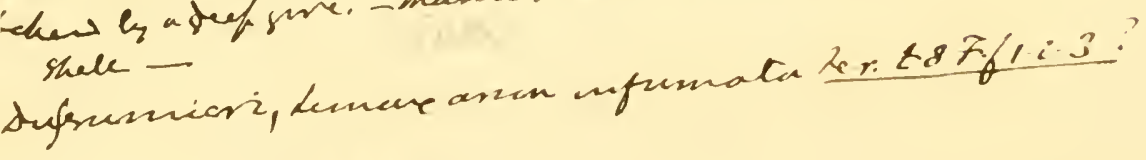

Panmancerarip

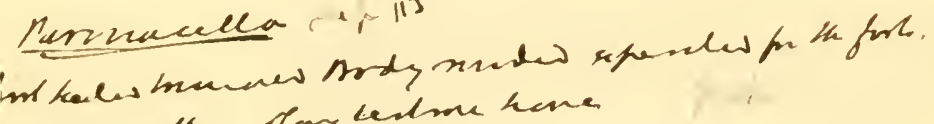

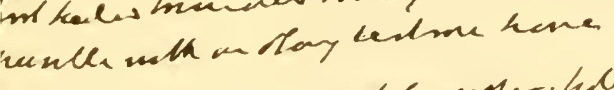

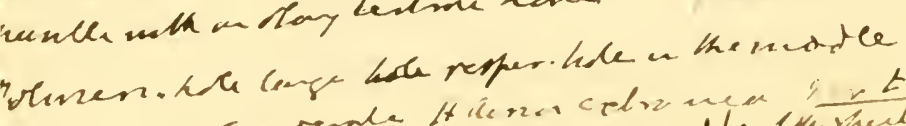

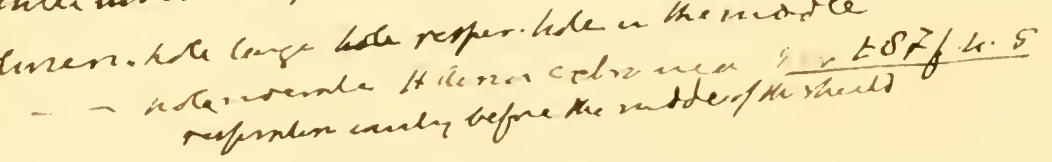


4. Icris, n. 39:2=Cenia, Alder of Hanc.

1. I. Cocksii. Cenia C., Alder \&. Hanc. t. 177. f. 1.

5. Fl cola, 11. 389.

1. F. rubra, Quoy, t. 180. f. 13.

Fam. 9. Sagitade, p. 164.

1. SAgITA, n. 339.

2. Sagitella, 11. 340. 1. S. xquipinnis, Lesueur.

\section{Order V. Pneuniobraxchiata.}

Respiring free air in a chamber lined with pulmonic ressels. Larva shell-bearing, without any cephalic fins; shaped like the parent.

\section{Puenuato Suborder I. ADELOPNEUMONA. ohofuenduta, i 64}

Edge of mantle unted to the nape, corering the pulmonary cavity, cal impregnation. Operetim iroma

A. Geophila. Eyes at apex of elongated cylindrical peduncles; $/ \mathrm{kcal}$ tentacles cylindrical, shorter, and under eye peduncle, sometimes wanting. Operculum none. Torrestrial.

a. Eye-peduncles and tentacles retractile under the skin.

Fam. 1. Arionida, p. 16.9.

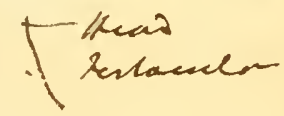

Caudal gland distinct.

1. Arion, n. 429.

1. Arion ater. A. empericorum, Ferussac, t. 281 . f. 1, t. 285 . f. 11.

A. albus, Feruss. t. 281 . f. 3. A. mufus, Feruss. t. 284. f. 5.

2. A. subfuseus, Drap. t. 276 . f. 10.

3. A. hortensis, Feruss. t. 280 . f. 2 , $\beta$. t. 280 . f. 8.

4. 1. fuscatus, Feruss. t. 285 . f. 8.

5. A. ascensionis, Quoy, t. 287. f. 11.

6. A. lactescens. Limacellus 1., Blainv. t. 285. f. 10.

2. Helicarion, n. 431.

1. H. Cuvieri, Feruss. t. 287 . f. 1.

2. H. Freycineti, Fernss. t. 287. f. 2 ; Blainv. t. 71 . f. 4 .

3. Strenopus, n. 433. Edge of mantle produced, forming a collar and partly covering the shell.

1. S. cruentatus, Guild. t. 72 . f. 2,4 ; Swainson, t. 288. f. 4.

2. S. - - Guild. t. 288. f. 5.

4. Nanina, n. 433 , pars.

1. N. vitrinoides, Gray, Hardwicke MSS. t.71. f. 5.

2. N. citrina. Vitrina c., Quoy, t. 7l. f. 7.

3. N. viridis. Vitrina v., Quoy, t. 7l. f. 3.

4. N. flammulata. Vitrina f., Quoy, t. 7l. f. 1.

5. N. nigra. Vitrina n., Quoy, t. 71. f. 2.

6. N. - ? Vitrine couverte, Eydoux, t. 288, f. 3. 
5. Arioninaxta, Desmoulin. "Edge of mantle inclosed, not pronlueed."

1. A. lavipes, Desmoulin, t. 288. f. 6, 7.

Fam. 2. Helicida, p. 170.

Caudal gland noue.

$$
\text { a. Philomycina. }
$$

1. Prilomisers, n. 434. Landalle $M$

1. P. carolinensis. Limax c., Bosc. t. 285. f. 4.

2. Meghimatum, n. 435 .

1. II. - - Ferussac, t. 276. f. 3.

2. II. - Ferussac, t. 276 . f. 6 .

3. JANELLA. Mantle corering the back; respiratory orifiec in middle of right side. 'Tentacles 2 , elongate, lower' wanting.

1. J. bitentaculatus. Jimax b., Quoy, t. 180. f. 15 .

4. Limax, n. 436.

b. Limaeina.

1. L. antiquorum, Ferussac, t. 280 . f. 3, t. 281 . f. 2, t. 284. f. 4; Mr. E. Gray, MSS. t. 280 . f. 5.

2. L. cincreo-niger, Sturm, t. 276 . f. 9.

3. L. alpinus, Ferussac, t. 280 . f. 6.

4. L. variegatus, Ferussac, t. 285. f. 12.

5. L. arrestis, Ferussac, t. 285 . f. 7.

6. I. Gagates, Ferussac, t. 280 . f. 4.

7. L. marginatus, Drap, t. 276. f. 4.

8. L. sylvaticus, Drap. t. 276. f. 7.

9. L. carinatus, Soxerby, t. 284 . f. 6.

10. L. Sowerbii, Ferussac, t. 276 . f. 8 .

11. 1. valentianus, Ferussac, t. 280 . f. 7 .

12. L. bilobatus, Ferussac, t. 285 . f. 9.

13. I. megaspidus, Blainville, t. 285. f. 5.

14. I. sanitwichensis, Eydour, t. 288 . f. 1.

15. L. canariensis, D'Orb. t. 284 . f. "2.

16. L. carinatus, $D^{\prime} O r b$. t. 284 . f. 3 .

17. I.? perlucidus, Quoy, t. 74 . f. 7.

18. L. ? noctilucus, Ferussac, t. 285 . f. 6.

5. Geomalaces, 11. 437.

MPi. (x. maenlatus, Mr. E. Gray, MSS. t. 280. f. 1.

c. T'estacellina.

6. Plectropholus, n. 439.

1. P. Orbignii, Ferussac, t. 285 . f. 3.

2. P. costatus, Bosc. t. 285 . f. 2.

3. P. carinatus, Bosc. t. 285 . f. 1 .

7. 'Testacella, n. 440.

1. 'T. haliotoidca, Ferussac, t. 283. f. 3 ; Mr. E. Gray, MSS. t. 283.

f. 6 ; Cuvier, t. 283. f. 1 ; Sowerby, t. 283. f. 7.

2. T. Mnugci, Ferussuc, t. 2\&3. f. 4. 


d. Vitrinina.

8. Parmacella, n. 441.

1. P. Olivieri, Cuvier, t. 282. f. 5 ; Eichwald, t. 282. f. 1. 122 nde

2. P. Valencienii, $W e b b$, t. 282 . f. 2.

3. P. palliolum, Ferussac, t. 282 . f. 3.

4. P.? - Helicolimax, Ferussac, t. 286. f. 1, shell quite covered.

5. P. - - Helicolimax, Ferussac, t. 286. f. 4, shell partly exposed.

6. P. - - Helicolimax, Ferussac, t. 286. f. 2, shell partly cxposed.

9. Cryptella, n. 442.

1. C. ambigna. C. canariensis, $W^{e} b b$, t. 284. f. 1. Parmacella ambigua, Ferussac, t. 283 . f. 5.

10. VITRINA, n. 444.

1. V. pellucida, Fleming, t. 287. f. 5. Helicolimax p., Ferussac, t. 288 . f. 11; Sturm, t. 287. f. 6.

2. V. brevis. Helicolimax b., Ferussac, t. 288. f. 8 .

3. V. elongata. Helicolimax e., Ferussac, t. 288. f. 9.

4. V. vitrea. Helicolimax v., Ferussac, t. 288, f. 12.

5. V. fasciata, Eydoux, t. 288, f. 2.

6. V. fasciolata. Helicolimax f., Ferussac, t. 286. f. 7 .

7. V. - - Helicolimax, Ferussac, t. 286. f. 3.

8. V. - Helicolimax, Ferussac, t. 286. f. 5.

11. Helicophanta, n. 445 (printed Helicolimax).

1. H. lufa, Hartmann, t. 287 . f. 3.

2. H. brevipes, Ferussac, t. 228. f. 10, t. 287. f. I3; Hartmann, t. 287 . f. 4.

12. Omalonyx, n. 446. 1. O. unguis.

13. Helisigna, n. 448.

1. H. santæ helenæ, Lesson, t. 287. f. 12.

2. I. fragilis. Ambrette fragile, Eydonx, t. 301. f. 2.

14. Succinea, n. 449.

1. S. putris, Sturm, t. 287. f. 8; Swainson, t. 287. f. 7 ; Desh. t. 287 . f. 10.

15. HeLIX, n. 452 .

e. Helicina.

1. II. Pomatia, Rossmasler, t. 296. f. 8; Hartmann, t. 295. f. 1 ; Cuvier, t. 295. f. 4 ; Chiaje, t. 295. f. 5.

2. H. lucorum (?), Chiaje, t. 295. f. 2.

3. H. melanostoma, Ferussac, t. 289 . f. 6.

4. H. naticoides, Ferussac, t. 288. f. 13; Rossm. t. 294. f. 5 ; Chiaje, t. 290. f. 14 ?

5. H. candidissima, Ferussac, t. 298. f. 7.

16. Acavus, n. 453.

1. A. nemoralis. Helix n., Ferussac, t. 289.f. 8; Lister, t. 294. f. 3 ; Sturm, t. 294. f. 14; Rossm. t. 292. f. 3, t. 294. f. 2; Chiaje, t. 290 . f. $1 \& 5$.

2. A. constantina. Helix c., Ferussac, t. 293. f. 9, t. 298. f. 2 ; Sturm, t. 290. f. 11.

3. A. hortensis, Helix h., Hartm. t, 290, f. 10. 
4. A. austriaea. IIelix a., Rossm. t. 294. f. 12.

5. A. laetea. IIelix 1., Ferussac, t. 298. f. 6.

6. A. vermiculata. Helix v., Fernssac, t. 298. f.4; Rossm. t. 294. f.6.

7. 1. alonensis. IIelix a., Ferussac, t. 298. f. 5.

8. 1. splemdila. Helix s., Ferussac, t. 298. f. 1.

9. 1. nicensis. Helix 11., Ferussac, t. 296. f. 2.

10. A. aspersa. Ilelix a., Rossm.t. 296 . f. 13 ; Chiaje, t. 290. f.16?

\section{ARIANTA, n. 454.}

1. A. arbustorum. Helix a., Feruss. t. 96. f. 3, t. 298. f. 3; Hartm. t. 290 . f. 8; Rossm. t. 290. f. 4; Sturm, t. 294 . f. 13.

2. A. frutieum. Helix f., Rossm. t. 294. f. 1; Sturm, t. 294. f. 11.

3. A. eingulatum. Helix c., Rossm. t. 294. f. 9.

18. Vallonia, n. 456.

1. V. pulchella. IIelix p., Sturm, t. 296. f. 5 ; Binney, t. 292. f. 4.

2. V. cornea. Helix c., Sturm, t. 296. f. 7 .

3. V. pellis serpentis, Ferussac, t. 298. f. 8.

4. V. exclusa. Helix e., Quoy, t. 292. f. 2.

5. V. zonaria. Helix z., Quoy, t. 72. f. 1.

6. V. undulata. Ielix n., Quoy, t. 72. f. 3.

7. V. - Helice de Touranne, Eydoux, t. 303. f. 8.

19. IBERUS, 11.457.

1. I. Gaulterianus. Hclix G., Ferussac, t. 297. f. 1.

2. I. lapicidus. Ilelix 1., Rossm. t. 292. f. 4; Sturm, t. 296. f. 11.

3. I. Lamarckii. Helix L., Ferussac, t. 297. f. 2.

4. 1. oresiguns. Helix o., D'Orb. t. 292. f. 8.

5. I. acutus. Inelix a., Quny, t. 292. f. 7.

6. I. solarium. IIelix s., Quoy, t. 292. f. 9.

20. Polygira, n. 460.

1. P. albolabris. Helix a., Bimey, t. 292. f. 2.

2. P. major. IIelix in., Binney, t. 291. f. 1.

3. P. thyroideus. Inelix t., Binney, t. 291. f. 7.

4. P. zelete. Ilelix \%., Bimney, t. 2!) 1. f. 9.

5. P. dentifera. Helix d., Bimney, t. 291. f. 11.

6. P. elevata. Ilelix e., Binney, t. 29) f. 8.

7. P. subglobosa. IIelix s., Bimey, t. 291. f. 6.

8. P. pennsylvaniea. IIelix p., Bimey, t. 291. f. 5.

9. P. palliata. IIelix p., Binney, t. 2933. f. 8 .

10. P. profundat. Ilelix p., Bimey, t. 293. f. 11.

11. P. monolon. IIclix m., Bimey, t. 293. f. 12.

12. P. inflecta. Ilclix i., Bimey, t. 292. f. 7.

13. P. fraterna. Helix f., Bimney, t. 2(2). f. 5.

14. P. hirsuta. Helix lı, Bimney, t. 2!)3. f. 3.

15. P. tridentata. Holix t., Bimey, t. 291 . f. 3.

16. P. grularis. IIelix g., Bimmey, t. 29) f. f. 4.

17. P. obvoluta. IIelix o., Sturm, t. 296. f. 9. Trigonostoma o., Hartm. t. 290 . f. 15.

21. TrEBA, n. 46].

1. T. ericetornm. Helix e., Sturm, t, 294. f. 4.

2. T. strigata. Ilelix s., Ferussae, t. 296. f. 1.

3. T. quimperiana. Ilelix q., Ferussac, t. 289. f. 7. 


4. T. zonata. Helix z., Ferussac, t. 289. f. 5.

5. T. villosa. Helix v., Sturm, t. 296. f. 12.

6. T strigilla. Helix s., Sturm, t. 296. f. 6.

7. T. incarnata. Helix i., Sturm, t. 294. f. 8.

8. T. clandestina. Helix c., Hartm. t. 292. f. 5.

9. T. bicarinata. Helix b., Lowe, t. 295. f. 8, var. Lowe, t. 294. f. 9.

10. 'T. portosanctx, Lowe, t. 291 . f. 7.

11. T.? roseo-tincta. Helix r., Forbes, t. 292. f. 6.

22. Helicella, n. 462.

1. I. rotundata. Helix y., Sturm, t. 296. f. 4.

2. H. Lucida. Helix 1., Sturm, t. 290. f. 2.

3. H. cellaria. Helix c., Rossm. t. 296. f. 10.

4. H. nitida. Helix n., Sturm, t. 294. f. 7, 10.

5. H. algira. Helix a., Ferussac, t. 289. f. 9.

6. H. saxatilis. Delomphalus s., Hartm. t. 292. f. 6.

7. H. ammoniformis, $D^{\prime}$ Orb. t. 287. f. 9.

23. Drepanostoma, Porro, n. 457.

1. D. multiformis, Porro, t. 290. f. 12.

24. Streptaxis, 11.464.

1. S. contusa. Helix c., Ferussac, t. 289. f. 1.

2. S. - Hélice égarée, Eydoux, t. 289. f. 4.

3. S. - H. déviée, Eydoux, t. 289. f. 3.

25. Bulinus, 12. 466 .

f. Bulimina.

1. B. hæmastomus, Lister, t. 299. Helix h., Lesson, t. 300, f. 15.

2. B. oblongus, $D^{\prime} O r b$. t. $74^{*}$. f. 4 .

3. B. granulosus, D'Orb. t. 73. f. 8.

4. B. thamnoicus, D'Orb. t. 73. f. 6.

5. B. rosaceus, $D^{\prime} O r b$. t. 73 . f. 10.

6. B. Tupaeii, $D^{\prime} O r b$. t. $74^{*}$. f. 2.

7. B. heterotrichus, Moricaud, t. 302. f. 5 .

8. B. hygrohylaus, $D^{\prime}$ Orb. t. 73. f. 2.

9. B. helaoicus, $D^{\prime} \mathrm{Orb}$. t. 73 . f. 9.

10. B. aspodemetes, $D^{\prime} O r b$. t. 73 . f. $3 \& 5$.

11. B. trichodes, $D^{\prime} O r b$. t. 73. f. 4.

12. B. Montaguii, D'Orb. t. 72 . f. 5 .

13. B. linostomus, D'Orb. t. 73. f. 7.

14. B. cambus, $D^{\prime} O r b$. t. 73 . f. 1 .

15. B. lavis, Quoy, t. 74. f. I; Lamk. MSS. t. 293. f. 2.

16. B. - Helix peal de renard, Eydoux, t. 303. f. 4.

17. B. trilineatus, Quoy, t. 74. f. 2.

18. B. candidus, Moricand, t. 302 . f. 4.

19. B. Pantagruelinus, Moricaud, t. 302 . f. 3.

20. B. Spixii, D'Orb. t. 302. f. 7.

21. B. Taunaisi, Ferussac, t.

22. B. Maximilianus, Moricaud, t. 302. f. 6 .

23. B. undulatus. Plekocheilus 1., Guilding, t. 74*. f. 1 .

24. B. chilensis, Lesson, t. 74. f. 6.

25. B. radiatus, Sturm, t. 300. f. 11 .

26. B. - Moricaud, t. 300 . f. 14. 
26. PARTLLA, n. 467.

1. P. gibba. Helix gibba, Qnoy, t. 74. f. 4.

2. P. —. I'. de Dumartrog, Eydoux, t. 303. f. 6 .

27. \%us, n. $46 \mathrm{~s}$.

1. Z. tridens. Ageca t., Leach, Brit. Moll.

2. \%. lubrica, Sturm, t. 300. f. 4.

2R. Buliaveus, M. 470.

1. 13. montams, Sturm, t. 300. f. 10. Ena m., Hartm. t. 300. f. 6.

2. 13. - Swainsom, t.300. f. 13.

3. 13. turritellus, Eydoux, t. 3013. f. 3.

4. B. - 13. ombilique, Eydoux, t. 303. f. 2.

5. 13. decollatus, Ferussac, t. 286. f. 8.

6. 13. striatellıs, Bull. Sci. t. 302. f. 9.

7. B. hamnensis, Bull. Sci. t. 302. f. \&.

8. B. - Chiaje, t. 310. f. 3 .

9. B. reginus. Bulimus r., D'Orb, t. 74*, f. 3.

10. B. zigzag. Ilelix undata, var. t. $301, \mathrm{f} .4$.

11. B. Kambul. Kambul, Adanson, t. 74. f. 3, t. 301. f. 6.

29. PupA, n. $471=$ Cerium, Mus. Berl. 1818.

1. P. dolium, Sturm, t. 310. f. 4.

2. P. chrysalis, Ferussac, t. 286. f. 9.

30. VERTIGO, ก. 472.

1. V. - Leach, Malac. Brit. t.

31. Clausilia, n. 475.

1. C. bideus, Sturm, t. 300, f. 7 ; Müller, t. 300. f. 5 ; Rossm. t. $3(0)$. f. 3 .

2. C. similis, Rossm. t. 300 . f. 8 .

3. C. cochinchinensis, Eydoux, t. 303. f. l.

32. BALEA, n. 476 .

? 1. B. perversa. Helix p., Sturm, t. 300. f. 1.

33. BrachYTU', n. 477.

1. 13. costatus. Siphonostoma c., Swerinson, t. 300. f. 9.

2. B. - - Siphonostoma, Sucains. t. 300 . f.

g. Achatinima.

34. AcuAtiNa, n. 478 = Achathina, Latr. $1804=$ Achitinum, Wus. Berl.

1. A. mamiciana, Lesson, t. 75. f. 1 .

35. Colcmisa, 12. 479.

1. C. virgata. Ilelix Columma, Quoy, t. 302. f. 1.

36. Ingeres, n. 484.

l. L. alabaster. IJclix a., Qnoy, t. 30:2. f. 2. 


37. Oleacina, n. 485 .

1. O. truncata. Glandiua t.,Wyman, t. 301 . f. 5.

2. O. Poiretii. Helix P., Ferussac, t. 301. f. 1.

3. O.? aperta. Macrospira a., Swainson, t. 300. f. 2.

38. Helicteres, n. 487.

1. H. decorata. Helix d., Eydoux, t. 103. f. 5.

b. Eye peduncle and tentacula contractile only.

Fam. 3. Veronicellide, p. 178. heth oordo

1. Veronicella, 1.488.

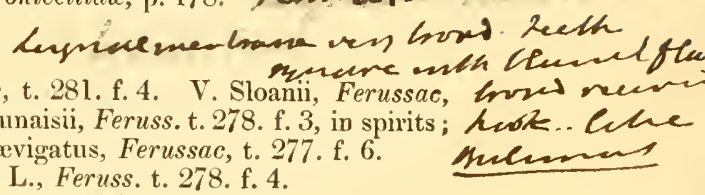

2. V. Langsdorfii. Vaginulus L., Feruss. t. 278. f. 4

3. V. Solea. Vaginulus S., D'Orb. t. 278. f. 2.

4. V. Limayana. Vaginulus L., Lesson, t. 278. f. 1 .

5. V. Krausii. Vaginulus K., Feruss. t. 276. f. 2.

6. V. - - Vag. de Luçon, Eydoux, t. 277. f. 5.

7. V. - - Vag. de Tonranne, Eydoux, t. 277. f. 3.

8. V. - - ? Vaginulus, sp., Ferussac, t. 278 . f. 5 ; t. 279. f. 1, 2, $3,4,5,6$; t. 276 . f. $1 \& 5$.

Fam. 4. Onchidiada, p. 179.

1. ONCHidum, n. 489.

1. O. Typhæ, Buchanan, t. 181. f. 8, t. 182. f. 2.

2. Buchannia, n. 490 .

1. B. onchidioides, Lesson, t. 214. f. 3.

3. Peronia, n. 491. Back with radiating processes.

1. P. mauriciana, Blainv. t. 183. f. 2. Onchidium Peronii, Cuvier, Pìe shafued cenv t. 181. . 7 .

2. P. ferrugineum, Lesson, t. .f. .

3. P. Alderi. P. punctata, Alder, MSS. t. 226. f. 3.
4. P. - Onchidium Peronii, Savigny, t. 183 . f. 4 .

neth os serente

5. P. punctata. Onchidium p., Quoy, t. 183. f. 3.

6. P. Tongensis. Onch. de Tongo, Quoy, t. 182. f. I.

4. Onchidelua. Back without radiating processes. Leth 00.00

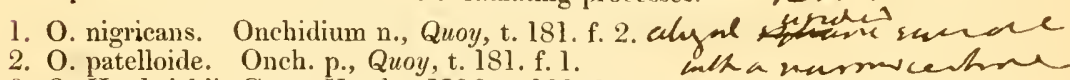

3. O. Hardwickii, Gray, Hardw. MSS. t. 182. f. 3. r. r. k.

4. O. granulosa. Onchidium g., Lesson, t. 183. f. 1.

5. O. nigra. Onchidium n., Lesson, t. 183. f. 7.

6. O. Reevesii, Gray, Reeve's MSS. t. 181. f. 5.

7. O. marmorata. Onchidium m., Lesson, t. 181. f. 6.

8. O. cinerea. Onchidium c., Quoy, t. 181. f. 3.

9. O. incisa. Onchidium i., Quoy, t. 181, f. 4.

10. O. nana. Onchidium n., Philippi, t. 214. f. 2. 
в. Limmophila. Eyes sessile. Tentacula 2, subcylindrical or flattened; simply contractile. Operculum none.

Fam. 5. Auriculide, p. 179.

Eyes on nape at the inner sides of base of the tentacle.

* Tentacles subcylindrical.

1. AURICULA, n. 492.

1. A. Midx, Quoy, t. 306. f. 12.

2. A. Judx, Eydoux, t. 304. f. 4.

2. MelaMpus, n. 493.

1. MI. - Mr. E. Gray, MSS. t. 305. f. 2.

2. Ml. lutens. Aur. 1., Quoy, t. 306. f. 5.

3. II. fuseus. Aur. brume, Eydonx, t. 304. f. 1.

3. CASSIDUla, n. 494.

1. C. auris felis. Aur. a. f., Eydonx, t. 304. f. 3.

2. C.? monilis. Aur. m., Quoy, t. 306. f. 10, 11 .

3. C. xqualis. Melampus æ., Lowe, t. 305. f. 3.

4. Pentees, 11.495.

1. P. afra, Lowe, t. 305. f. 4. Pedipes, Adanson, t. 305. f. 5.

5. Ophicardelus, n. 497.

1. O. australis. Aur. anstralis, Quoy, t. 306. f. 8, 9.

2. O. sandwichensis. Lur. s., Eydoux, t. 304. f. 2.

6. Alexin, n. 498.

1. A. denticulata. Auricula a., Lowe, t. 305. f. 1. ? Voluta Dargelasii, Chiaje, t. 306. f. 4 .

7. Scarabus, n. 500 .

1. S. imbrium, Lesson, t. 306 . f. 3.

** Tentacles compressed, flattened.

8. Cimlina, n. 504.

1. C. fluctuosa. Dombeya f., D'Orb. t. 306. f. 1.

2. C. puelcha. Domb. p., D'Orb. t. 306. f. 2.

Fam. 6. Lymmearla, p. 180.

- Bujes orrinper sile of base of the flattengal or filiform tentaeles.

1. LyaneA, 11. 503. Tentacles flattened.

1. L. stagnalis, Cuvier, t. 30R. f. 5 ; Rossm. t. 308. f. 13 ; Desh. t. 308. f. 12; Chiuje, t. 308. f. 10 ; Sturm, t. 308. f. 9. Stagnicola vulgaris, Hartm. t. 3018 . f. ¿.

2. I. plalustris, Lister, t. :308. f. 1 ; Cuvier, t. 308. f. 8 .

3. L. caperata, Hartm. t. 310. f'. \&.

4. L. fragilis, Hartm. t. 310. f. 6.

5. L. oahoneusis, Eydoux, t. 304. f. 5. 


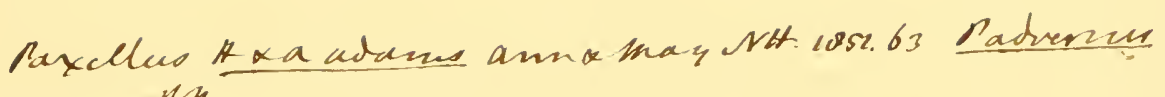
mish

otina its

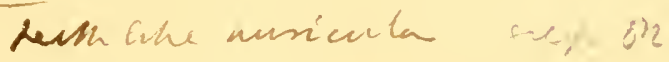

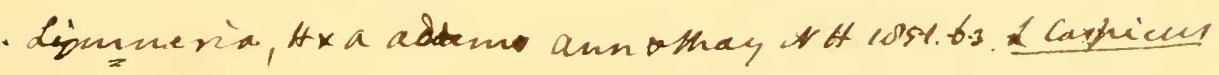






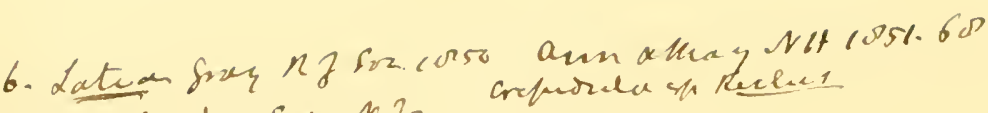

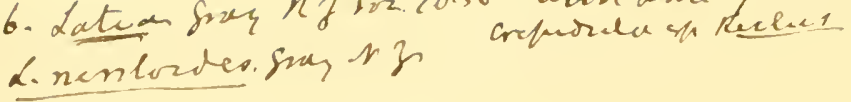


6. L. Catoscopium, Hartm. t. 310 . f. 7.

7. L. ovalis, Cuvier, t. 308. f. 2 ; Chiaje, t. 308. f. 4.

8. L. pereger, Hartm. t. 308. f. 6; Sturm, t. 308. f. 7 .

9. L. auricularia, Sturm, t. 307. f. 1 .

10. L. Lessouii, Lesson, t. 307 . f. 2.

2. AMPHIPEPLEA, n. 505 .

1. A.? involuta, Beaufort's MSS. t. 301. f. 3.

2. A. luconensis, Eydoux, t. 304. f. 7 .

3. Bulinus, n. 506=Aplexa, n. 507 .

1. B. Adansonii. Bulin, Adanson, t. 306. f. 7.

2. B. Guildingii. Diastropha, Guilding, t. 307. f. 3.

3. B. affinis. Lymnée voisine, Eydoux, t. 304. f. 6.

4. B. hypnorum. Physa h., Sturm, t. 307. f. 6.

4. Physa, n. 506, pars. Bulinus is a syn. of Aplexa.

1. P. fontinalis, Hüller, t. 307 . f. 5 ; Sturm, t. 307 . f. 4.

2. P. heterostropha, Haldem. t. 310 . f. 9 .

5. Coretus, n. 508.

1. C. Adansonii. Coretns, Adanson, t. 309. f. 4.

2. C. corneus. Planorbis c., Sturm, t. 309. f. 14; Rossm. t. 309. f. 13; Swains. t. 309. f. 12; Desh. t. 309. f. 11; Lister, t. 309. f. 9 ; Cuvier, t. 309 . f. 2 .

3. C. bicarinatus. Planorbis b., Haldem. t. 310. f. 1 .

4. C. contortus. Planorbis c., Sturm, t. 309. f. 6.

5. C. hispidus. Planorbis h., Sturm, t. 309. f. 7.

6. C. carinatus. Planorbis c., Sturm, t. 309. f. 5.

7. C. vortex. Planorlois v. Sturm, t. 309. f. 1.

8. C. Spirorbis. Planorbis S., Sturm. t. 309. f. 3.

9. C. fontanns (distorted). Hippeutes lenticularis, Hartm. t. 309. f. 8 .

10. C. dubius. Planorbis d., Hartm. t. 309. f. 10.

6. Seguentina, n. 509 .

1. S. ? armigera. Planorbis a., Say, t. 310. f. 2.

7. Ancrlus, n. 510.

1. A. fluviatilis, Sturm, t. 307. f. 7-10.

2. A. radiatus, Guilding, t. 307 . f. $6^{*}$.

3. A. lacustris, Sturm, t. 307 . f. 8 .

4. A. rivularis, Haldemann, t. 310 . f. 5.

5. A. Sayanus. A. radiatus, $D^{\prime} O r b$. t. 307 . f. 9 .

c. Thalassophila. Eyes sessile, on front part of the frontal disk Reat 70 trithon formed by the expanded tentacula. — Relloro. oo

Fam. 7. Siphonariada, p. 181. Operculum none. Shell conic. Latín anforme

1. SIPHONARIA, n. 512.

1. S. - Savigny, t. 275. f. 1 .

2. S. diemensis, Quoy, t. 76. f. 1.

3. S. algesiræ, Quoy, t. 76. f. 2.

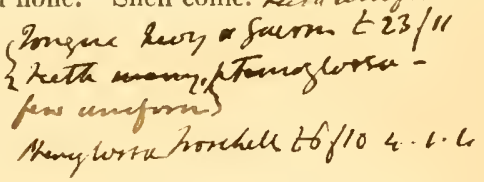


4. S. viridis, Quoy, t. 76. f. 3.

5. S. albicans, Quoy, t. 76. f. 4 .

6. S. uustralis, Quoy, t. 76. f. 5 .

7. S. acuta, Quoy, t. 76. f. 6.

8. S. capensis, t. 76 . f. 7 .

9. S. Lessonii, D'Orb. t. 275 . f. 33.

10. s. picta, D'Orb. t. 275 . f. 2.

nuencelouse Fam. 8. Amplibolide, p. 181. Opereulum and shell spiral. erowat

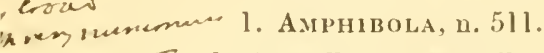

1. A. avellana. Ampullacera a., Quoy, t. 310. f. 12.

2. A. anstralis. Imp. fragilis, Quoy, t. 310. f. 11 \& 13.

mopenalatu $h$ b4 Suborder II. Phaneropnedioxa.

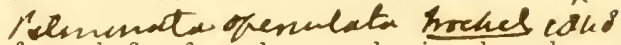

a - Elge of mantle free from the nape, leaving the pulmonary carity open. Animal unisexual. Operculum distinct, spiral, or anuulated.

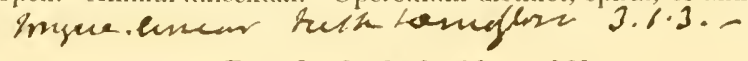
Fam. 9. Cyclophorida, p. 181.

Eyes sessile, on the onter side of the hase of the tentacula. Operculuin spiral.

1. Crclostosus, n. 514.

1. C. elegans, Mr. E. Gray, MSS. t. 293. f. 1; Rossm. t. 311. f. 16,17 ; Chiaje, t. 311.f. 14. f. 9? f. 5?

2. Crclotus, n. 520.

1. C. Inca. Cyclost. I, D'Orb. t. 311.

2. C.? troehiforme. Cyclost. t., Eydoux, t. 303. f. 11 .

3. C. ? anguliferus. Cyclost. a., Eydonx, t. 303. f. 9.

4. C.? - Cyclost. tacheté, Eydoux, t. 303. f. 7.

3. LEPTOPOMA.

1. L. multilahris. Cyelost. m., Quoy, t. 311. f. \&.

2. L. nova hibernia. Cyclost. 11., Quoy, t. 311 . f. 13.

4. Оторома.

1. O. Listeri. Cyclost. fimbriata, Quoy, t. 311 . f. 10.

5. REALIA, 11. 525.

l. R. ruhens. Cvelost. r., Quoy, t. 311.f. 11.

2. R. erosa. Cyclost. e., Quoy, t. 311. f. 12.

6. Ponatias, n. 529.

1. P. maculata. Cyclost. m., Sturm, t. 311. f. 19.

7. Megalomastoma, n. 524.

1. M. brunnea. Cyclost, b., Swuinson, t. 311. f. 15. 


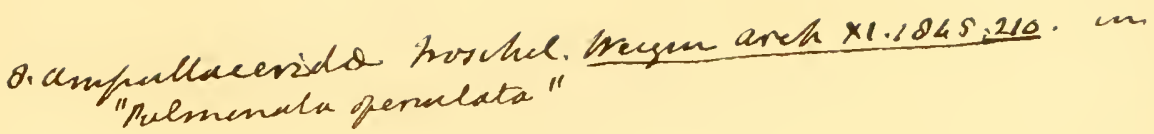

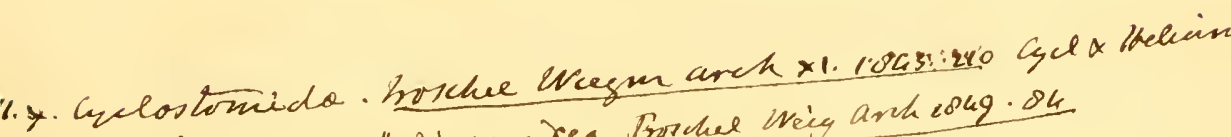

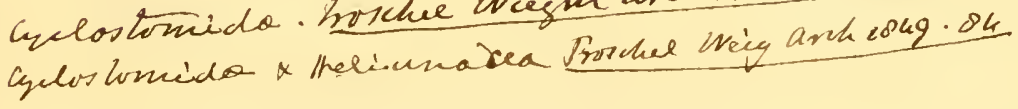



Fam. 10. Oligyrude, p. 182.

Eyes on outer side of the base of the tentacula. Opcreulum amuilar.

1. Oligyra, n. 531 .

1. O. flammea. Helicina f., Quoy, t. 311. f. 3.

2. O. tæniata. Helicina t., Quoy, t. 31 l. f. 1.

3. O. sandwichensis. Helicina s., Eydoux, t. 303. f. 10.

4. O. oresignæ. Helicina $0 ., D^{\prime} O r b$. t. 311. f. 6.

5. O. zephirina. Helicina z., Duclos, t. 311. f. 2.

6. O. variabilis, Guilding, t. 290. f. 9, t. 311. f. 7 .

7. O. occidentalis. Helicina o., Guilding, t. 311. f. 4.

2. Alcadia, n. 533.

3. Steganostoma, Adums.

\section{Fam. 11. Aciculada.}

Tentacula subulate. Eyes on the hearl, rather behind and between the tentacula. Operculum horny, spiral, of few rapidly enlarging whorls. Foot divided across (allied to Truncatella and Auricula).

1. Acicula, Hartmann $=$ Acmea, n. 502=Pupula, Hartmann $=$ Truncatella, Adams. Aperture simple.

1. A. fusca. Pupa lineata, Hartmann, t. 123. f. 9.

2. A. striata. Cyclostoma s., Quoy, t. 123. f. l.

2. Geomelania, Pfeiffer = Cyclostoma, sp. Quoy. Outer lip expanded, sinuous. 1. G. jamaicensis, Pfeiffer.

\section{Class IV. PTEROPODA.}

Arranged according to GRAY's Catalogue of Pteropoda in the Collection of the British Museum, 1850.

Order I. Tinecosonata.

A. Body and shell struight. Operculum none.

Fam. 1. Cavolinida.

1. Cavolina, Gray's Cat. Pterop. p. 5. (Hyalæa, n. 765.)

1. Cavolina tridentata. Anomia t., Forsk. t. 78. f. 10, t. 244. f. 2. Hyalea cornea, Chiaje, t. 78. f. 9. H. Forskalii, D'Orb. t. 78 . f. 7, t. 244.f. 11, t. 252. f. 3. H. tridentata, Blainv. t. 78. f. 11; Eydoux, t. 243. f. 2; Cuv.t. 244. f. 3, 5, 8; Van Beneden, t. 244.

f. 4. Canlinia natans, Poli, t. 44. f. 1, 2, t. 244. f. 4, 6, 7. H. australis, Lesueur, t. 244. f. 1 ; ? Hooker, t. 245. f. 1, $2,6$.

2. C. tæniobranchia. Hvalæa t., Lesueur, t. 243. f. 5.

3. C. affinis. Hyalrea a., D'Orb. t. 78. f. 3.

4. C. gibbosa. Hralæa g., D'Orb. t. 78. f. 2 ; Eydoux, t. 243. f. 10.

5. C. uncinata. Hyalæa 1 ., D'Orb. t. 78. f. 5 ; Eydoux, t. 243. f. 6.

6. C. flava. Hyaliea f., D'Orb. t. 78. f. 4 . 
7. C. globulosa. Hyalara g., Eydonx, t. 243. f. 3.

8. C. angulosa. Hyalixa a., Eydoux, t. 243. f. 1.

9. C. quadridentata. Hyalea q., D'Orb. t. 78. f.6; Eydoux, t. 243. f. 7 ; Hooker, t. 245 . f. 3 .

10. C. longirostra. IIyalxa l., D'Orb. t. 7R. f. 1; Eydoux, t. 243. f. 4 ; Quoy, t. 77. f. 12.

11. C. limbata. Il valiea l., D’Orb. t. 244. f. 10.

12. C. labiata. Hyalax 1., D'Orh. t. 77. f. 1 ; Eydoux, t. 243. f. 9.

13. C. inflexa. Ilyalixa i., D'Orb.t.77.f. 3; Eydoux, t. 243. f. 11 ; Hooker, t. 24s. f. 4.

2. Dracria, n. 767. Gray, Cat. Pterop. 10.

1. D. trispinosa. Ifyalxa t.. Quoy, t. 77. f. 13; D'Orb. t. 77. f. 6, t. 129. f. 3; Eydoux, t. 246. f. 1.

2. D. mucronata. II yalaea m., D'Orb. t. 77. f. 2, var.?; Cuvier, in R. A. Croch. t. 17. f. 6.

3. D. depressa. IIyalxa d., D'Orb. (t. 7. f. 11, 14).

4. D. lavigata. Hyalaca 1., D'Orb.t. 78. f. 8; Eydoux, t. 243. f. 8.

3. Clio, n. 768.

1. C. pyramiclata. Cleodore p., Lesueur, t. 246. f. 10; Hooker, t. 218 . f. 6 , ? f. 3, t. 247. f. 6 . Hyalar p., $D^{\circ}$ Orb. t. 77. f. 4 ; Cantraine, t, 247. f. 5. II. lanceolata, Lesueur, t. 247. f. 4; Quoy, t. 247.f. 3; Eydoux, t. 246. f. 7. Cleorlore de Bronn, Blaiuv. t. 77. f. !.

2. C. cuspidata. Cleodore c., Quoy, t. 247. f. 2; D'Orb. t. 77. f. 5; Cantraine, t. 247. f. 1; Eydoux, t. 246. f. 2. C. quadrispinosa, Lessou, t. 247. f. 11, animal fictitious.

3. C. Lamartinieri. Tar. or n. s. 1. Hooker, t. 248. f. 2. 2. Hooker, t. 248 . f. 1 .

4. C. depressa, Eydoux, t. 246. f. 3.

5. C. Chaptalii, Eydonx, t. 246. f. 6.

6. C. pellucidi. Pleuropus p., Eschsch. t. 249. f. 7. Cleodore coubée, Eydoux, t. 246. f. 8.

4. BALA.NTIUM, 11. 769 .

1. B. recurvmu. Cleodora balantium, Rung, t. 247. f.9. Hyalaea b., D'Orb. t. 247 . f. 10.

2. B. intlatum. Cleodora i., Eydoux, t. 246. f. 4; Hooker, t. 248. f. 5. Var. and n. s. Hooker, t. 218. f. 7.

3. B. australis. Cleodore a., I'Orb.t. 250. f. 8; Eydoux, t. 246. f. 5.

4. B. rugosum. IIyaliea $x, D^{\prime} O, b$. t. 247 . f. 7.

5. STYLIOLA =Cresis, 12.772 .

$$
\text { * Mouth angular. Cresis. }
$$

1. S. subula. Cresis spiniferi, Rung, t. 251. f. 5, t. 77. f. 11. Cleodora sulula, Eydoux, t. 25l. f. 12; Quoy, t. 251. f. 9. Hyalæa s., D'Orb. t. 2.'

$$
\text { ** Houth circular. Styliola. }
$$

2. S. virgula. Cresis v., Rung, t. 25l. f. 1. Clcoulore r., Eydoux, t. 250. f. 7, t. 251. f. 11. Creseis caligula, Eschsch. t. 249. f. 4. Crescis unguis, Eschsch. t. 24\%. f. 2. Creseis cornucopia, Eschsch. t. 249. f. 6. Hyalaca v., D'Orb. t. 77. f. 8; Hooker. t. 250 . f. 1 . 


3. S. corniformis. Hyalæa c., D'Orb. t. 77. f. 7.

4. S. recta. Cresis aciculata, Rang, t. 251. f. 2. C. clava, Rang, t. 251. f. 4. Cleodore aciculata, Eydoux, t. 251. f. 7. Creseis acus, Eschsch. t. 249. f. 5 ; Hooker, t. 250. f. 3?, 5, 6.

4*. S.? —? Creseis —, Eschsch. t. 249. f. 1 .

*** Mouth compressed.

5. S. striata. Cresis s., Rang, t. 251. f. 6. Hyalæa s., D'Orb. t. 77. f. 10. Cleodore s., Eydoux, t. 247. f. 8. ? Creseis compressa, Eschsch.t. 249. f. 3; ? Hooker, t. 250, f. 2, 4.

Fam. 2. Tripterida.

1. Triptera, n. 781, Gray, Cat.Pterop. 28, and Cuvieria, n. 780.

1. T. columnella. Cuvieria c., Rang, t. 252. f. 2. C. columella, D'Orb. t. 252. f. 1.

2. T. rosea, Quoy, t. 258. f. 12.

Fam. 3. Cymbuliada.

1. Crmbulia, n. 782.

1. C. proboscidea, Peron, t. 252 a.f. 2. C. Peronii, Eydoux, t. $252 a$. f. 1; Rang, t. 252 a.f. 3 ; Cantr. t. 251 a.f. 7 ; Blainv. t. $251 a$. f.6, animal reversed; Desh.t.252. f.6; Van Beneden, t.252. f.5.

2. C. ovata, Quoy, t. 251 a. f. 5.

3. C. radiata, Quoy, t. 251 a. f. 4.

4. C. punctata, Quoy, t. 251 a.f. 1.

5. C. Norfolkensis, Quoy, t. 251 a. f. 2.

2. Euribia, n. 774 .

1. E. hemisplıærica, Rang, Ann. Sci. Nat. xii. 1827, t. 45 ß. f. 9.

2. E. Gaudichaudi, t. 246 . f. 9.

3. Psyche, n. 773.

1. P. globulosa, Rang, t. 259. f. 2.

4. ThedemaNia, n. 783.

1. T. neapolitana. Cymbulia n., Van Beneden, t. 252. f. 4. T. creniptera, Krohn, t. 252. f. 5.

В. Body and shell spiral. Operculum distinct.

Fam. 4. Limacinida.

1. Limacina, n. 777 .

1. L. arctica, Scoresby, t. 259. f. 4. Clio helicina, Fabricius, t. 259.

f. 1. Spiratella limacina, Blainv. t. 259. f. 4.

2. L. antarctica, Hooker, MSS. t. 259. f. 3, 5, 6 .

3. L. inflata. Spirialis rostralis, Eydoux, t. 260. f. 1.

4. L. rotunda. Atlanta 1., D'Orb. Toy. Amer. Mer. t. 12. f. 20, 24.

5. L. ventricosa. Spirialis v., Eydoux, t. 260. f. 2.

6. L. anstralis. Spirialis a., Eydoux, t. 260. f. 4.

7. L. trochiformis. Spirialis trochiformis, Eydoux, t. 260. f. 6.

8. I. Bulimoides. Spirialis Bulimoide, Eydoux, t. 260. f. 5. 


\section{Order II. Grinosomata.}

1. Clione, n. 789.

Fam. 5. Clioneida.

1. C. borealis, Pallas, t. 258. f. 1. Clio arctica, Fabricius, t. 258.

f. 5. Clio b., Scoresby, t. 258. f. 4, from life; Cuvier, t. 258.

f. 2; Eydoux, t. 252. f. 1. C. miquelonensis, Rang, t. 132. f. 7.

2. C. anstralis, Blainv. t. 257 . f. 2.

3. C. caudata, Eydoux, t. 253. f. 2.

2. Cliodita, n. 790 .

1. C. caduceus, Quoy, t. 258. f. 7 .

2. C. fusiformis, Quoy, t. 258. f. 6.

3. C. pyramidalis, Quoy, t. 258. f. 3.

Fan. 6. Pneumodermide.

1. PNeunoderion, n. T84.

1. P. cucullata, Peron \& Lesueur, t. 253. f. 4, t. 269. f. 2; Cuvier,

t. 257. f. 4. C. Peronii, Bluinv. t. 257. f. 5 ; Eydoux, t. 253.

f. 3; Quoy, t. 257 . f. 1 .

2. P. luber, Quoy, t. 256. f. 3. Var. Quoy, t. 256. f. 2.

3. P. pellucida, Quoy, t. 256. f. 1 .

4. P. violacea, D'Orb. t. 256. f. 5. Clio capensis, Quoy, t. 132. f. 8.

2. Spongiobrancilea, n. $7 \$ 5$.

1. S. anstralis, D'Orb. t. 256. f. 4; Hooker, t. 258 . f. 9.

2. S. elongata, D'Orb. t. 256. f. 11.

3. - n. s. t. 10. f. 9.

3. Trichocycles, n. 786. 1. 'T. Dinmerillii, Eschsch. Isis, 1837, t. .f. .

4. Pelagia, 1. 787. 1. P. alloa.

Fam. 7. Cymorloceada.

1. CYMODOCEA.

1. C. diaphana, D'Orb. t. 258 . f. 10 ; ? Hooker, t. 258. f. 8.

TIIE END.

\section{Curded.}

PHINTED BY RICHARD AND JOIIN E. TAYLOR, IED LION COURT, FLEET STREET. 






$1 \%$ 


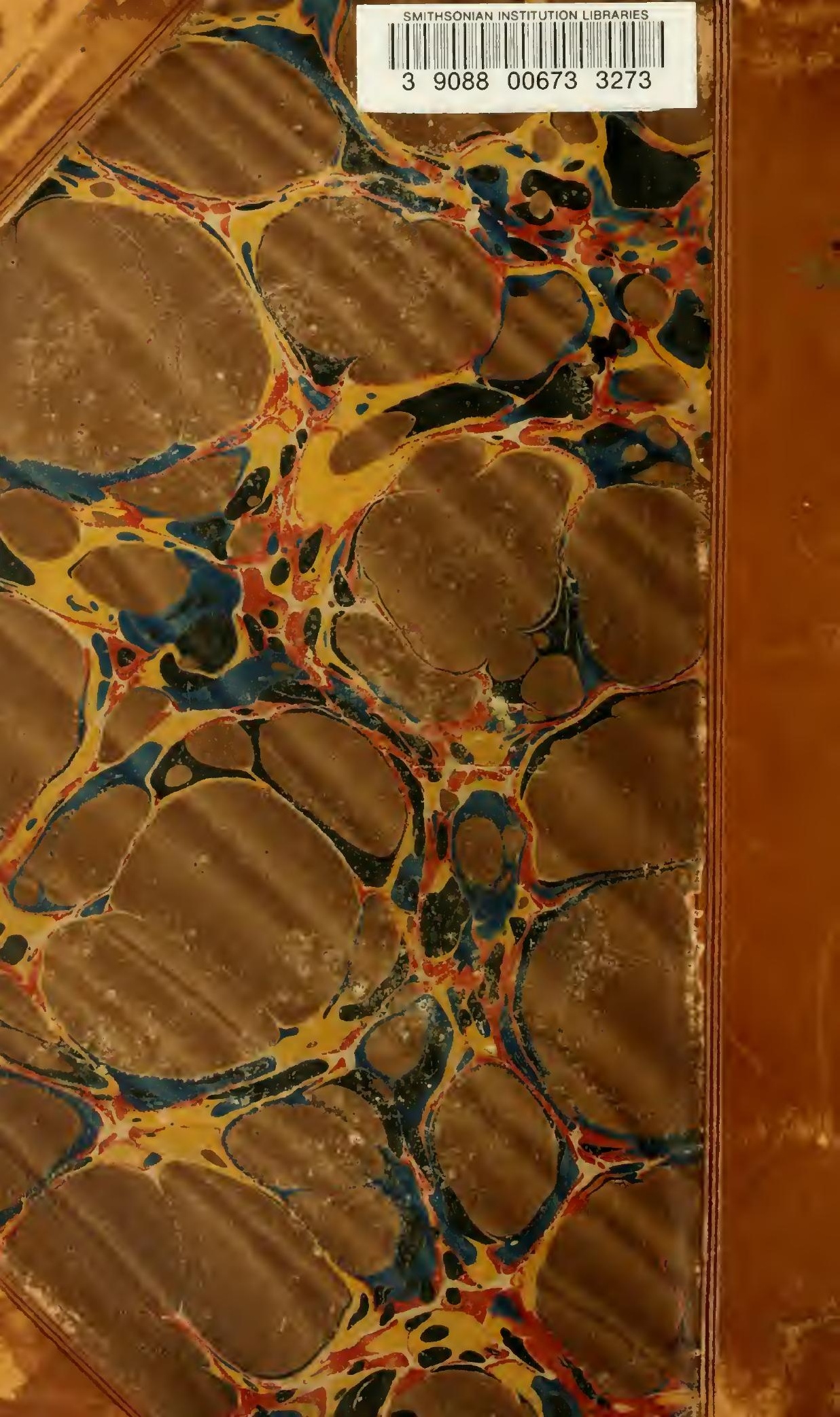

